Draft Version March 4, 2022

Preprint typeset using $\mathrm{LATE}_{\mathrm{E}} \mathrm{X}$ style emulateapj v. 03/07/07

\title{
CHEMICAL INHOMOGENEITIES IN THE MILKY WAY STELLAR HALO
}

\author{
IAN U. ROEDERER \\ Department of Astronomy, University of Texas at Austin \\ 1 University Station C1400, Austin, TX 78712-0259 \\ Draft version March 4, 2022
}

\begin{abstract}
We have compiled a sample of 699 stars from the recent literature with detailed chemical abundance information (spanning $-4.2 \lesssim[\mathrm{Fe} / \mathrm{H}] \lesssim+0.3$ ), and we compute their space velocities and Galactic orbital parameters. We identify members of the inner and outer stellar halo populations in our sample based only on their kinematic properties and then compare the abundance ratios of these populations as a function of $[\mathrm{Fe} / \mathrm{H}]$. In the metallicity range where the two populations overlap $(-2.5 \lesssim[\mathrm{Fe} / \mathrm{H}] \lesssim$ $-1.5)$, the mean $[\mathrm{Mg} / \mathrm{Fe}]$ of the outer halo is lower than the inner halo by $\sim 0.1$ dex. For $[\mathrm{Ni} / \mathrm{Fe}]$ and $[\mathrm{Ba} / \mathrm{Fe}]$, the star-to-star abundance scatter of the inner halo is consistently smaller than in the outer halo. The $[\mathrm{Na} / \mathrm{Fe}],[\mathrm{Y} / \mathrm{Fe}],[\mathrm{Ca} / \mathrm{Fe}]$, and $[\mathrm{Ti} / \mathrm{Fe}]$ ratios of both populations show similar means and levels of scatter. Our inner halo population is chemically homogeneous, suggesting that a significant fraction of the Milky Way stellar halo originated from a well-mixed ISM. In contrast, our outer halo population is chemically diverse, suggesting that another significant fraction of the Milky Way stellar halo formed in remote regions where chemical enrichment was dominated by local supernova events. We find no abundance trends with maximum radial distance from the Galactic center or maximum vertical distance from the Galactic disk. We also find no common kinematic signature for groups of metal-poor stars with peculiar abundance patters, such as the $\alpha$-poor stars or stars showing unique neutron-capture enrichment patterns. Several stars and dSph systems with unique abundance patterns spend the majority of their time in the distant regions of the Milky Way stellar halo, suggesting that the true outer halo of the Galaxy may have little resemblance to the local stellar halo.

Subject headings: Galaxy: formation - Galaxy: halo - globular clusters: general — nuclear reactions, nucleosynthesis, abundances — stars: abundances — stars: Population II
\end{abstract}

\section{INTRODUCTION}

The nucleosynthesis reactions necessary to produce metals in stars were realized nearly half a century ago (Burbidge et al. 1957; Seeger et al. 1965; Fowler et al. 1967; Wagoner et al. 1967), yet the challenge to interpret the wide variety of nucleosynthetic signatures observed in different stellar populations today remains as intriguing as ever. Metal-poor stellar populations should contain recycled stellar material from fewer generations of stars than metal-rich populations, making interpretation of their chemical enrichment history-in principle-simpler. Thanks to numerous large surveys over the last four decades designed to identify metal-poor stars (see review by Beers \& Christlieb 2005), the tally of known metal-poor stars now stretches well into the thousands. Concurrently, great advances have been made in the analysis and interpretation of the chemical signatures and enrichment histories revealed by stellar spectra (e.g., Audouze \& Tinslev 1976, Kraft 1979, Wheeler et al. 1989, McWilliam 1997, Gratton et al. 2004, Beers \& Christlieb 2005, and Sneden et al. 2008). Furthermore, careful laboratory analysis has improved our knowledge of the relevant atomic data necessary to make accurate and detailed records of the chemical composition of the atmospheres of metal-poor stars. The confluence of advances in each of these fields has built upon the foundation of stellar nucleosynthesis to greatly increase our understanding of the earliest generations of stars, the chemical evolution of the various components

Electronic address: iur@astro.as.utexas.edu of the Milky Way Galaxy, and the formation process of the Galaxy, to name just a few successes.

Correlations between metal enrichment in stellar populations and the kinematic properties of these stars have been known for some time now (e.g., Eggen et al. 1962; Wallerstein 1962), and through the years these relationships have been fleshed out in increasing detail. Most studies of the formation of the stellar halo of the Galaxy have employed limited chemical data (e.g., $[\mathrm{Fe} / \mathrm{H}]^{1}$ ) to accompany the kinematic data. This is sufficient to study the metallicity distribution function (MDF) of the halo (e.g., Hartwick 1976; Rvan \& Norris 1991b; Ivezić et al. 2008; Schörck et al. 2008), formation and age of the halo (e.g., Eggen et al. 1962; Searle \& Zinn 1978; Sandage 1986; Wyse \& Gilmore 1988; Gilmore et al. 1989; Preston et al. 1991; Ryan \& Norris 1991a; Maiewski 1992; Norris 1994; Carney et al. 1996; Sommer-Larsen et al. 1997; Chiba \& Yoshii 1998; Chiba \& Beers 2000; Carollo et al. 2007; Bell et al. 2008; Miceli et al. 2008; Morrison et al. 2008), or for investigating stellar streams and halo substructure (e.g., Majewski et al. 1996; Helmi \& White 1999; Chiba \& Beers 2000; Gilmore et al. 2002; Kinman et al. 2007). Iron ( Fe) - or, in some cases, calcium (Ca) - alone is less useful for studies of the chemical enrichment of the halo, which can examine, e.g., supernova (SN) models and rates, stellar binary fractions, mixing processes in the interstellar medium (ISM) of the halo, the Galactic

1 We adopt the usual spectroscopic notations that $[\mathrm{A} / \mathrm{B}] \equiv \log _{10}\left(\mathrm{~N}_{\mathrm{A}} / \mathrm{N}_{\mathrm{B}}\right)_{\star}-\log _{10}\left(\mathrm{~N}_{\mathrm{A}} / \mathrm{N}_{\mathrm{B}}\right)_{\odot}$ and $\log \epsilon(\mathrm{A}) \equiv$ $\log _{10}\left(\mathrm{~N}_{\mathrm{A}} / \mathrm{N}_{\mathrm{H}}\right)+12.00$ for elements $\mathrm{A}$ and $\mathrm{B}$. 
potential, Galactic structure, and relationships between various substructures (e.g., globular clusters, Local Group dwarf spheroidal [dSph] systems, stellar streams). It was not until recently that kinematic and detailed chemical data for halo stars were analyzed together (e.g., Gratton et al. 2003; Simmerer et al. 2004; Venn et al. 2004; Pritzl et al. 2005; Font et al. 2006; Geisler et al. 2007).

Three substantial advances have been made in the short period of time since Venn et al. (2004) and Pritzl et al. (2005) performed a detailed chemical comparison between field stars in the halo and nearby dSph systems and globular clusters. First, echelle spectrographs on multiple large $(6-10 \mathrm{~m}$ class $)$ telescopes have enabled investigators to carry out detailed abundance analyses of large numbers of (often faint) metal poor stars, including many stars with $[\mathrm{Fe} / \mathrm{H}]<-3.0$. Second, investigators at the US Naval Observatory have released revised proper motion catalogs based on longer time baselines, improved quality of astrographs, and better techniques for digitizing photographic plates (e.g., Zacharias et al. 2004a). Improved proper motions are given in these catalogs for many of the metal-poor stars investigated in recent years, enabling us to derive their full space motions through the Galaxy. Finally, large numbers $\left(\sim\right.$ few $\left.\times 10^{4}\right)$ of calibration stars with known stellar parameters and metallicities have been observed as part of the Sloan Digital Sky Survey (SDSS) and Sloan Extension for Galactic Understanding and Exploration (SEGUE) projects (e.g., Newberg et al. 2003; Allende Prieto et al. 2007; Lee et al. 2007). This has enabled the previously known kinematic properties of nearby members of the so-called inner and outer stellar halos to be assessed with a new level of detail (Carollo et al. 2007). These advances insist on a fresh reanalysis of the existing data. The goal of the present study is to interpret the wealth of recent high-resolution abundance analyses of metal-poor stars in light of the most recent kinematic knowledge of these two major components of the Galactic halo.

\section{ABUNDANCE DATA FROM THE LITERATURE}

Venn et al. (2004) compiled from existing literature a large sample of stellar abundances and $U V W$ kinematic data, when available, to compare the chemical enrichment patterns of metal-poor Galactic halo stars with present-day $\mathrm{dSph}$ systems. We adopt their data from the high-resolution abundance analyses of Edvardsson et al. (1993), Nissen \& Schuster (1997), Hanson et al. (1998), Prochaska et al. (2000), Fulbright (2000, 2002), Stephens \& Boesgaard (2002), Bensby et al. (2003), and Reddy et al. (2003). The Milky Way thin and thick disks are represented in this sample along with the halo, totaling 620 stars.

We supplement this sample with abundances derived from more recent high-resolution analyses of metal-poor halo stars or older studies that did not include any kinematic analysis. We add another 309 stars from the analyses of McWilliam et al. (1995a, b), Hill et al. (2002), Ivans et al. (2003), Cavrel et al. (2004), Honda et al. (2004a,b), Barklem et al. (2005), Honda et al. (2006), Francois et al. (2007), and Lai et al. (2008). For stars without sufficient kinematic information to compute $U V W$ velocities and Galactic orbital parameters (i.e., only the stellar radial velocity is published), we obtain proper motions from the catalogs listed in $\S 3.2$ Many of these stars are not in the Hipparcos Catalog (Perryman \& ESA 1997), and for these stars we derive a photometric parallax (see $\S 3.1$ ). We also require that the total proper motion is greater than 2.5 times its error, otherwise we discard the star from further kinematic analysis. We insert this requirement to derive accurate space velocities, yet unfortunately this will also bias our sample against stars with small proper motions; see $\S 5$ for closer analysis of this point. This requirement culls our sample by 230 stars. Our final sample of stars is given in Table 1 along with their adopted $[\mathrm{Fe} / \mathrm{H}]$ values.

This large dataset will allow us to probe abundance trends as a function of stellar kinematics. Because we mix data from different sources, systematic offsets in the abundance ratios arise from to a variety of factors, including (1) different spectral resolution and signal-tonoise $(\mathrm{S} / \mathrm{N})$ ratios of the data themselves, leading to potential blending of lines in crowded spectral regions, since high resolution is desirable to more-fully resolve the absorption line profiles yet such high resolution and high $\mathrm{S} / \mathrm{N}$ is impractical for studies of very faint stars; (2) different sets of absorption lines and transition probabilities used in each study; (3) different methods employed to determine stellar parameters; (4) differences in the structure of the model atmospheres themselves; and so on. These sources of systematic error should be no larger than $0.1-0.2$ dex in $[\mathrm{X} / \mathrm{Fe}]$, which does limit our ability to detect subtle chemical signatures; gross trends should still be identified reliably. At any given $[\mathrm{Fe} / \mathrm{H}]$, our sample is comprised of stars from a variety of studies, and the characteristic scatters of the inner and outer halo populations are distinct beyond this level of systematic scatter.

\section{KINEMATIC DATA}

\subsection{Distance Estimates}

Hipparcos (Perryman \& ESA 1997) geometric parallaxes are the preferred method for deriving accurate distances for our sample (using the new reduction of the Hipparcos data described in van Leeuwen 2007). For stars not in the Hipparcos catalog, we derive distance estimates from the photometric parallax by comparing the observed $V$ magnitude with a predicted absolute magnitude $M_{V}$, derived from the dereddened $V-K$ color. $^{2}$ To establish the relationship between color and absolute magnitude, we use the $Y^{2}$ set of theoretical isochrones (Demarque et al. 2004). We select the appropriate isochrone for each star, assuming $[\alpha / \mathrm{Fe}]=$ +0.3 for all stars and choosing a metallicity close to the individual stellar $[\mathrm{Fe} / \mathrm{H}]$. We find that the choice of (old) age has negligible effect on our derived photometric distances (comparing between 10, 11, 12, and 13 Gyr isochrones), so we adopt an age of 12 Gyr for all stars. To break the degeneracy of $M_{V}$ with $V-K$ (since any given isochrone is not single-valued in $M_{V}$ for each value of $V-K$ ), we establish the evolutionary state of each star based on the surface gravity estimate given in the literature ("dwarfs": $\log g \geq 4.0$; "giants": $\log g<4.0)$. We adopt $V$ magnitudes from SIMBAD if

2 This method was only used to derive distances to stars with $[\mathrm{Fe} / \mathrm{H}]<-1.0$. 
not given in the references in $\S 2, K$ magnitudes from 2MASS $^{3}$ (Cutri et al. 2003), reddening estimates from the dust maps of Schlegel et al. (1998) and the reddening laws of McCall (2004): $R_{V}=3.070 \times E(B-V)$ and $R_{V-K}=2.727 \times E(B-V)$. We use the prescription given by Bonifacio et al. (2000b) to reduce the Schlegel et al. (1998) estimates for $E(B-V)>0.10$ : $E(B-V)_{\text {revised }}=0.10+0.65\left[E(B-V)_{\text {S98 }}-0.10\right]$.

Figure 1 compares our photometric and geometric distances for stars in Venn et al. (2004). In order to assess the reliability of our photometric distances alone, we attempt to minimize the effect of other influences on the result by restricting this comparison to stars with $E(B-V)<0.30$ and $[\mathrm{Fe} / \mathrm{H}]<-1.0$. The difference in the distance computed by the two methods is shown as a function of $V-K$ color, $[\mathrm{Fe} / \mathrm{H}]$ metallicity, and Hipparcos parallax. While it is apparent that there is poor agreement for the distance estimates for our giants, there exists no trend between the differences and color or metallicity. There is, however, a very clear correlation between these differences and the Hipparcos parallax. In other words, the agreement is very good for stars with reliable Hipparcos parallax measurements, but this correlation breaks down for stars with $\pi_{\text {Hipparcos }} \lesssim 5$ mas $\mathrm{yr}^{-1}$. (cf. Figure 1 of Chiba \& Yoshii 1998, $§ 3.3 .5$ and Figure 3 of Beers et al. 2000). This preferentially affects distant stars (giants, in our sample) that have parallax measurement uncertainties comparable to the size of the parallaxes themselves. The vast majority of stars with $\pi_{\text {Hipparcos }}>5$ mas $\mathrm{yr}^{-1}$ are dwarfs, but the few giants with large parallaxes exhibit generally good agreement between the two distance estimates. We also show the relative differences as a function of distance in the bottom panel of Figure 1. The scatter in the giants $(\sigma=0.59,7$ stars) is about twice as large as the scatter in the dwarfs $(\sigma=0.29,28$ stars $)$, which is to be expected since a given uncertainty in $(V-K)_{0}$ will translate to a relatively larger uncertainty in $M_{V}$ for stars on the giant branch than for stars on the main sequence or near the turn-off. As we proceed, we calculate geometric distances if the Hipparcos parallax is greater than 5 times its uncertainty, otherwise we estimate distance from the photometric parallax. These distances are reported in Table1

\subsection{Space Velocities}

We calculate $U V W$ space velocities for the stars in our sample from measurements of the radial velocity, proper motion, and distance to each star. We adopt a left-handed coordinate system, where positive $U$ denotes velocity towards the Galactic anti-center $\left(\ell=180^{\circ}\right.$, $b=0^{\circ}$ ), positive $V$ denotes velocity parallel to the direction of rotation of the Local Standard of Rest (LSR; $\ell=90^{\circ}, b=0^{\circ}$ ), and positive $W$ denotes velocity perpendicular to the plane of the disk of the galaxy $\left(b=+90^{\circ}\right)$. We correct for the motion of the Sun with respect to the LSR, adopting $\left(U_{\mathrm{LSR}}, V_{\mathrm{LSR}}, W_{\mathrm{LSR}}\right)_{\odot}=(-9$, 12, 7) (Mihalas \& Binnev 1981), where all velocities are measured in $\mathrm{km} \mathrm{s}^{-1}$. We adopt $V_{\mathrm{LSR}}=220 \mathrm{~km} \mathrm{~s}^{-1}$

3 This publication makes use of data products from the Two Micron All Sky Survey, which is a joint project of the University of Massachusetts and the Infrared Processing and Analysis Center/California Institute of Technology, funded by the National Aeronautics and Space Administration and the National Science Foundation.
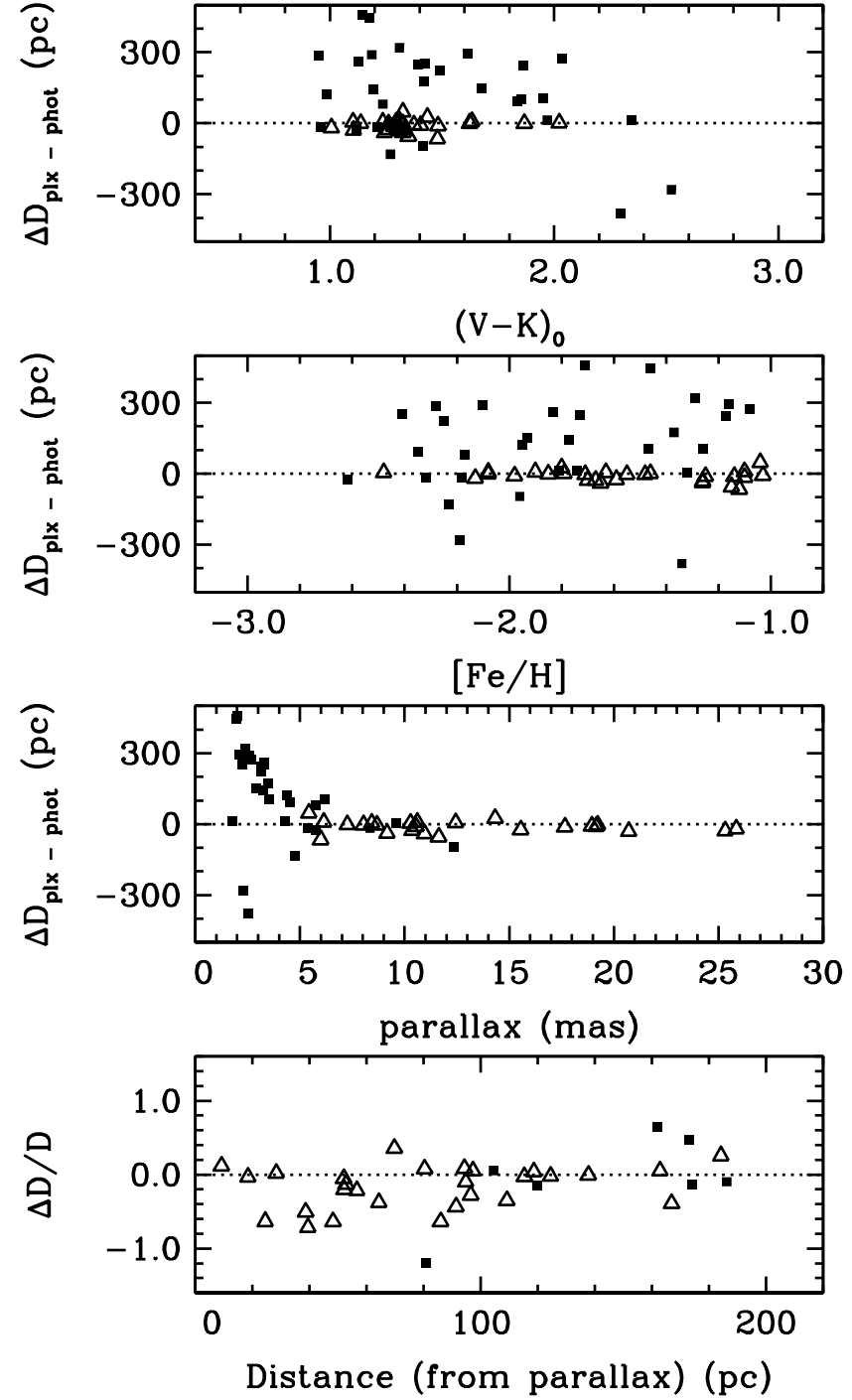

FIG. 1.- Comparison of distances derived from Hipparcos parallax measurements and photometric parallaxes, as a function of $(V-K)_{0},[\mathrm{Fe} / \mathrm{H}]$, and Hipparcos parallax. The bottom panel shows the relative differences as a function of distance (as derived from the Hipparcos parallax) for stars with $\pi_{\text {Hipparcos }}>5$ mas yr ${ }^{-1}$. Open triangles represent our dwarf sample $(\log g \geq 4.0)$ and filled squares represent our giant sample $(\log g<4.0)$.

(Kerr \& Lvnden-Bell 1986) and report the $V$ component in a Galactic reference frame (i.e., where the Sun's motion is described by $[U, V, W]=[-9,232,7])$. Radial velocities were taken from the literature references described in $\S 2$. Proper motions were taken from the second data release of the USNO CCD Astrograph Catalog (UCAC2; Zacharias et al. 2004a; Urban et al. 2004) or the USNO-B astrometry catalog ${ }^{4}$ (Monet et al. 2003), with preference given to the UCAC2 measurements.

The $U V W$ velocities, measured in a Cartesian coordinate system, can be transformed into a cylindrical coordinate system $(R, \phi, z)$ with its origin at the Galactic center, where $R=\sqrt{\left(R_{\odot}+x\right)^{2}+y^{2}}, \phi=\arctan \left(y /\left(R_{\odot}+\right.\right.$ $x)$ ), and $z=z$. Our adopted and derived $U V W$ and $V_{\phi}$ velocities are given in Table 1 .

\subsection{Galactic Orbit Parameters}

4 Accessible through the Naval Observatory Merged Astrometric Dataset, NOMAD (Zacharias et al. 2004b). 
We use the orbit integrator developed by D. Lin to calculate the Galactic orbital parameters for stars in our sample. The gravitational potential of the Galaxy is computed by summing the individual contributions of the disk, spheroid (or bulge), and halo components:

$$
\begin{aligned}
\Phi_{\text {disk }} & =\frac{-G M_{\text {disk }}}{\sqrt{R^{2}+\left(a+\sqrt{z^{2}+b^{2}}\right)^{2}}} \\
\Phi_{\text {spheroid }} & =\frac{-G M_{\text {spheroid }}}{r+c} \\
\Phi_{\text {halo }} & =v_{\text {halo }}^{2} \ln \left(r^{2}+d^{2}\right),
\end{aligned}
$$

where $R=\sqrt{x^{2}+y^{2}}$ and $r=\sqrt{x^{2}+y^{2}+z^{2}}, M_{\text {disk }}=$ $10^{11} \mathrm{M}_{\odot}, M_{\text {spheroid }}=3.4 \times 10^{10} \mathrm{M}_{\odot}, v_{\text {halo }}=128 \mathrm{~km} \mathrm{~s}^{-1}$, $a=6.5, b=0.26, c=0.7$, and $d=12.0$. This model is described in detail in Johnston et al. (1996) and Johnston (1998), who chose values for these parameters to reproduce a nearly-flat rotation curve for the Milky Way Galaxy between $r=1$ and $r=30 \mathrm{kpc}$ and a disk scale height of $0.2 \mathrm{kpc}$. We require that each star complete a minimum of 20 orbits around the Galactic center in 10 Gyr (at which point the orbital parameters have settled to a constant value); no orbital parameters are given in Table 1 for stars that did not meet this requirement. From the output we calculate $r_{\text {peri }}$ and $r_{\text {apo }}$, the minimum and maximum Galactocentric radii reached by each star; $\left|Z_{\max }\right|$, the maximum distance above (or below) the Galactic plane; and $e$, the eccentricity of the orbit, defined as $\left(r_{\text {apo }}-r_{\text {peri }}\right) /\left(r_{\text {apo }}+r_{\text {peri }}\right)$. These properties are also reported in Table 1

Our kinematic results ought to be reasonably independent of our model for the Galactic potential. Since we adopt the Galactic orbital properties of the inner and outer halo populations from Carollo et al. (2007) (see $\S$ (4), we want to demonstrate some degree of consistency between results derived from the two different potentials. To assess this, we compare the Galactic orbital properties of stars computed using Equation 3.3 to those computed using the analytic Stäckel-type potential (Sommer-Larsen \& Zhen 1990; Chiba \& Yoshii 1998) adopted by Beers et al. (2000) and Carollo et al. (2007). The parameters of their model have been tuned to reproduce a flat Galactic rotation curve beyond $R=$ $4 \mathrm{kpc}$ and the local mass density at the Solar radius. We use our three-component model of the Galactic potential to compute Galactic orbital parameters for 100 stars from the sample of Beers et al. (2000), strictly using their input data (distance, radial velocity, proper motion, etc.). Figure 2 compares the orbital parameters presented in Beers et al. (2000) (i.e., computed with the Stäckel-type potential) with those derived with our three-component model. Both models generally produce similar apogalactic and perigalactic distances as well as maximum vertical distance, but with a fair degree of scatter in each of these quantities. For $R_{\text {apo }}>10 \mathrm{kpc}$ and $\left|Z_{\max }\right|>2 \mathrm{kpc}$, the distances for individual stars are typically different by less than a factor of two. These systematic differences are inherent to the representations of the potential.

How worrisome are these differences? Our approach to identifying the two populations isolates them from one another in kinematic space by placing a buffer between them-reasonable uncertainty in the distance estimate or proper motion of a star should not cause it to drift from one classification to the other. For example, stars in our inner halo population must not orbit more than $15 \mathrm{kpc}$ from the Galactic center, while stars in our outer halo population must orbit to at least $25 \mathrm{kpc}$. We also adopt membership standards for the inner halo that rely on more than one kinematic selection criterion. Therefore, we do not consider this systematic difference a serious problem in our kinematic selection criteria.

\section{NEW KINEMATIC DEFINITIONS OF THE INNER AND OUTER HALO POPULATIONS}

Analyzing more than 10,000 calibration stars from low resolution $(R \equiv \lambda / \Delta \lambda \sim 2000)$ spectra obtained with the SDSS, Carollo et al. (2007) reported that the Milky Way stellar halo is roughly divisible into two broadlyoverlapping components. Their "inner halo" is composed of stars on highly eccentric orbits, has a flattened density distribution, exhibits a modest net prograde rotation, and has a peak in the MDF at $[\mathrm{Fe} / \mathrm{H}]=-1.6$. Their "outer halo" is composed of stars with a variety of orbital eccentricities, has a spherical density distribution, exhibits a net retrograde rotation, and has a peak in the $\mathrm{MDF}$ at $[\mathrm{Fe} / \mathrm{H}]=-2.2$. While their inner halo primarily dominates the stellar halo at Galactocentric radii $\lesssim 10-15 \mathrm{kpc}$ and the outer halo primarily dominates at radii $\gtrsim 15-20 \mathrm{kpc}$, these two components overlap in the Solar neighborhood, disguising their distinct characteristics from being recognized by earlier, smaller samples of stars.

We define membership in the inner and outer halo populations based only on the kinematics of stars in our sample. These new criteria, based on the classifications sketched by Carollo et al. (2007), are shown in Figure 3 . For the remainder of the present study, we shall use the working definition that inner halo membership is characterized by prograde rotation about the Galactic center $\left(V_{\phi}>0 \mathrm{~km} \mathrm{~s}^{-1}\right)$, relatively small maximum vertical distance from the Galactic plane $\left(\left|Z_{\max }\right|<5 \mathrm{kpc}\right)$, relatively small maximum radial distance from the Galactic center $\left(R_{\text {apo }}<15 \mathrm{kpc}\right)$, and rather high orbital eccentricity $(e>0.5)$. A star must meet each of these criteria to be classified as a member of the inner halo. Outer halo membership is characterized by significant retrograde rotation about the Galactic center $\left(V_{\phi}<-150 \mathrm{~km} \mathrm{~s}^{-1}\right)$, relatively large maximum vertical distance from the Galactic plane $\left(\left|Z_{\max }\right|>10 \mathrm{kpc}\right)$, or relatively large maximum radial distance from the Galactic center $\left(R_{\text {apo }}>25 \mathrm{kpc}\right)$. We apply no explicit orbital eccentricity requirements for outer halo membership, although the criteria essentially select stars on relatively eccentric orbits since they are presently in the Solar neighborhood and yet must travel to great distances. In contrast to the inner halo membership, stars only need to possess one of these characteristics to be included in the outer halo population. While our selection criteria may eliminate genuine members of either population, they help to ensure that there is minimal contamination between the two populations.

Interestingly, our inner halo criteria may allow for the inclusion of a few thick disk stars on mildly eccentric orbits. To eliminate these stars, we adopt one additional criterion for membership in the inner halo. Assuming that the stellar thick disk and halo populations each have Gaussian velocity distributions in each of the three com- 

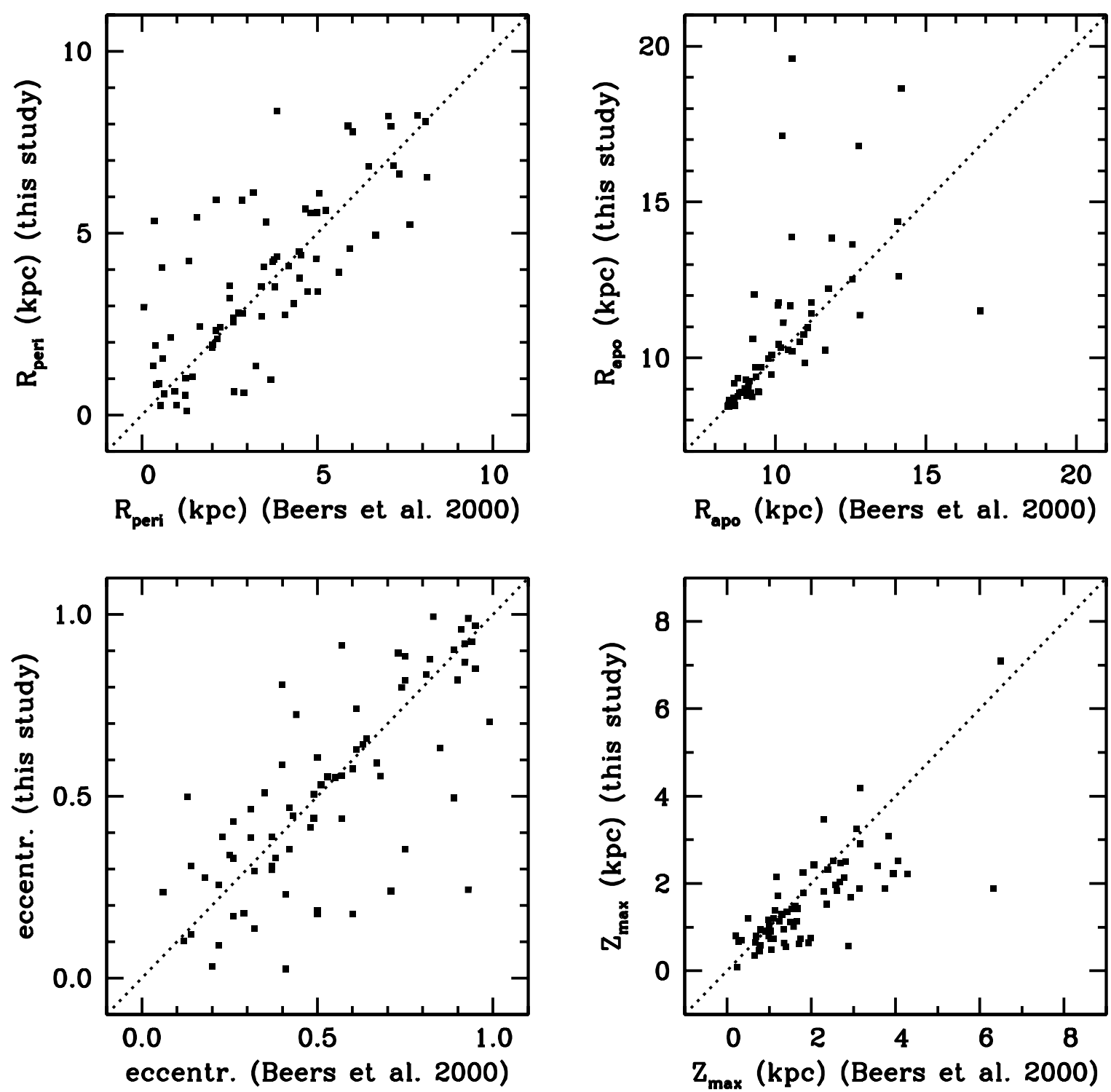

FIG. 2.- Comparison of our predicted Galactic orbit properties with those of Beers et al. (2000). Our values have been computed using the three-component model for the potential, while Beers et al. (2000) used the analytic Stäckel-type potential.

ponents (e.g., Bensby et al. 2003, eqns. 1 and 2), we calculate the relative probability that a star is in the thick disk versus the halo. We use the velocity dispersions $\left(\sigma_{U}, \sigma_{V}, \sigma_{W}\right)=(46,50,35) \mathrm{km} \mathrm{s}^{-1}$ for the thick disk and $(141,106,94) \mathrm{km} \mathrm{s}^{-1}$ for the halo, as well as the rotation speeds $\left\langle V_{\phi}\right\rangle=200 \mathrm{~km} \mathrm{~s}^{-1}$ and $\left\langle V_{\phi}\right\rangle=40 \mathrm{~km} \mathrm{~s}^{-1}$ for the thick disk and halo, respectively (Chiba \& Beers 2000). We require that inner halo members are at least twice as likely to be members of the halo than the thick disk. This eliminates less than $15 \%$ of the stars that would otherwise be classified as members of the inner halo.

Previous investigations (Stephens 1999; Stephens \& Boesgaard 2002; Fulbright 2002) have employed very similar criteria to ours for defining outer halo membership, including stars with retrograde Galactic orbits or probing to large Galactocentric distances in and above the plane of the disk. One unique feature of our study is the adoption of several selection criteria for defining membership in the inner halo, rather than simply identifying stars at less extreme maximum distances as inner halo members. This advance has been made possible because of large surveys (e.g., SDSS/SEGUE) that have identified very large numbers of metal-poor stars in the Solar neighborhood.

One abundance criterion-but not $[\mathrm{Fe} / \mathrm{H}]$ - is necessary to exclude stars whose abundances do not reflect their initial composition. When signatures of the slow neutron-capture nucleosynthesis process (" $s$-process"; see $\oint 6.3$ ) are enhanced in metal-poor stars, this is regarded as evidence of past mass transfer from an undetected companion star that passed through the asymptotic giant branch (AGB) phase of stellar evolution. Since the metal content of these stars has changed over the course of their lives, we exclude them membership in either halo population. ${ }^{5}$ Later, in $\S$ 8.2. we examine these stars for any common kinematic signatures.

5 The pure-s-process $[\mathrm{Ba} / \mathrm{Eu}]$ ratio is predicted to be $[\mathrm{Ba} / \mathrm{Eu}]_{\mathrm{s}}=$ +0.63 (Simmerer et al. 2004). We exclude stars with [Ba/Eu] > +0.5 and $[\mathrm{Ba} / \mathrm{Fe}]>+1.0$. Unfortunately, Eu has been measured in only $\approx 35 \%$ of the stars in our entire sample, so it is likely that some stars that have experienced $s$-process enrichment during their lifetimes will remain in our sample. Fortunately, though, nucleosyn- 

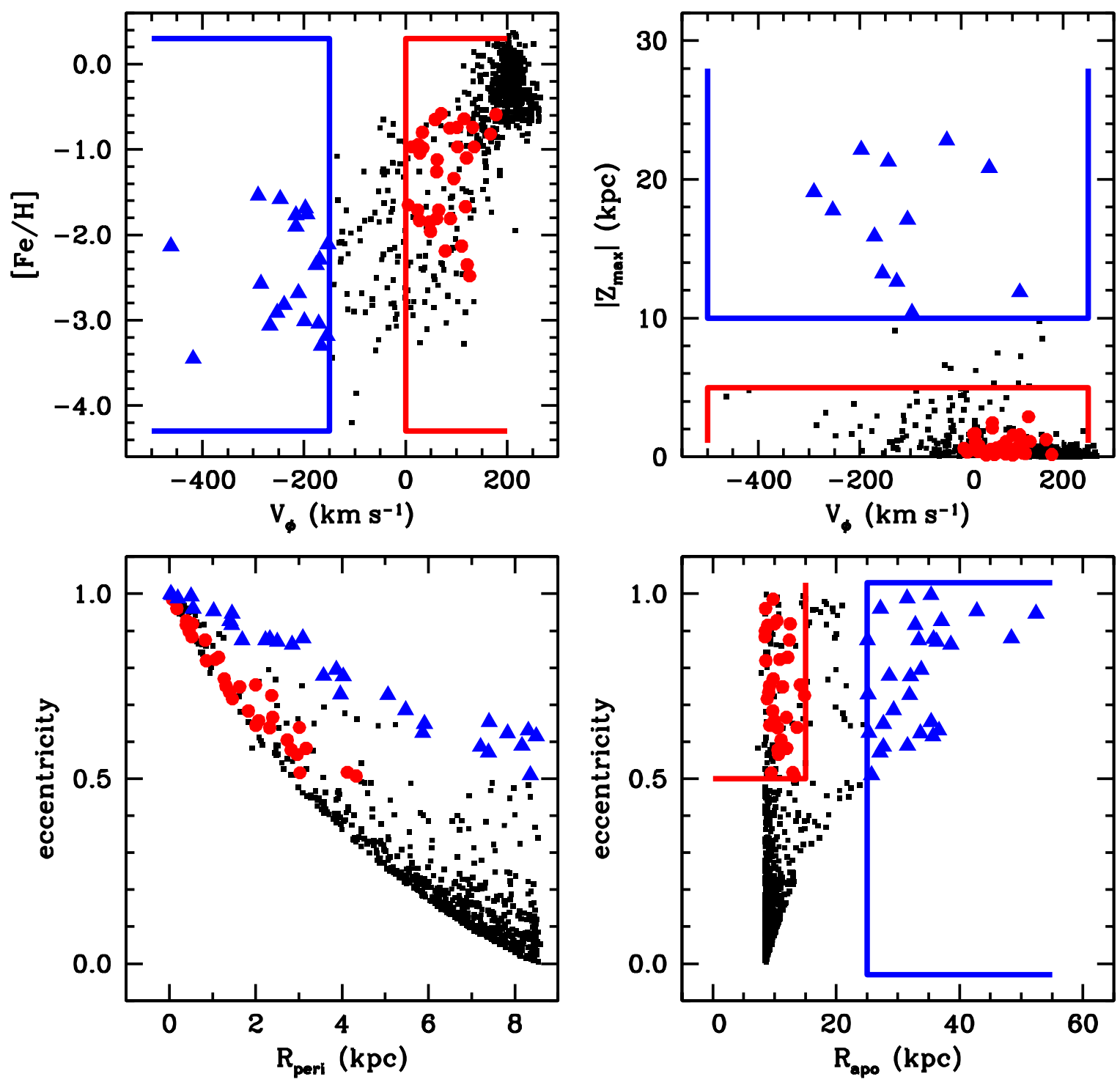

FIG. 3.- The kinematic definitions of our inner and outer halo samples. The inner halo population (dark gray circles, red in the online edition) is defined by stars that have $V_{\phi}>0 \mathrm{~km} \mathrm{~s}^{-1},\left|Z_{\max }\right|<5 \mathrm{kpc}, R_{\text {apo }}<15 \mathrm{kpc}$, and $e>0.5$. The outer halo population (black triangles, blue in the online edition) is defined by stars that have $V_{\phi}<-150 \mathrm{~km} \mathrm{~s}^{-1},\left|Z_{\max }\right|>10 \mathrm{kpc}$, or $R_{\text {apo }}>25 \mathrm{kpc}$. Stars that did not meet the kinematic criteria for these two populations are shown as small dots. [Please see the electronic edition for a color version of this figure.]

The members of both the inner and outer halo populations according to these definitions are listed, along with the abundance ratios we consider, in Tables 2 and 3.

\subsection{Classification Uncertainties}

In Figure 4 we examine the changes in the derived orbital parameters resulting from variations of the input measurements within reasonable uncertainties. We use the subsample of stars without prior kinematic analysis (the 309 stars noted in $\S 2$ ) for this test. The changes are obtained by adjusting the distance by $\pm 20 \%$, the proper motions by \pm 5 mas $\mathrm{yr}^{-1}$ (or $20 \%$ for small values of the proper motion), or the radial velocity by $\pm 10 \mathrm{~km} \mathrm{~s}^{-1}$. We also examine the effect of changing the mass of the Galaxy by $\pm 10 \%$. RMS values for each set of changes

thesis in the envelope of stars during the AGB phase has a negligible effect on most light elements, (C, N, O, F, Ne, and perhaps $\mathrm{Na}$ being notable exceptions; e.g., Straniero et al. 2006) including most of those examined in detail in our study. are shown on the right side of each panel in Figure 4 It is clear that, for most stars, these changes do not introduce appreciable uncertainty into the derived orbital parameters. For a small fraction of stars $(\approx 5 \%)$, the changes are large enough that they could cause an inner halo star to be classified as an outer halo star or vice versa. While some stars scatter in and out of the two populations, we confirm that no stars in this sample actually change their classification from inner to outer (or vice versa) with these variations in the input parameters. This result is a consequence of the multiple requirements necessary for inclusion in the inner halo population and the wide buffers placed between the kinematic and orbital properties of the two populations.

Confident that our definitions of the inner and outer halo populations are robust against uncertainties in the measurements, we proceed to compare the compositions of stars in the inner and outer halo populations. 

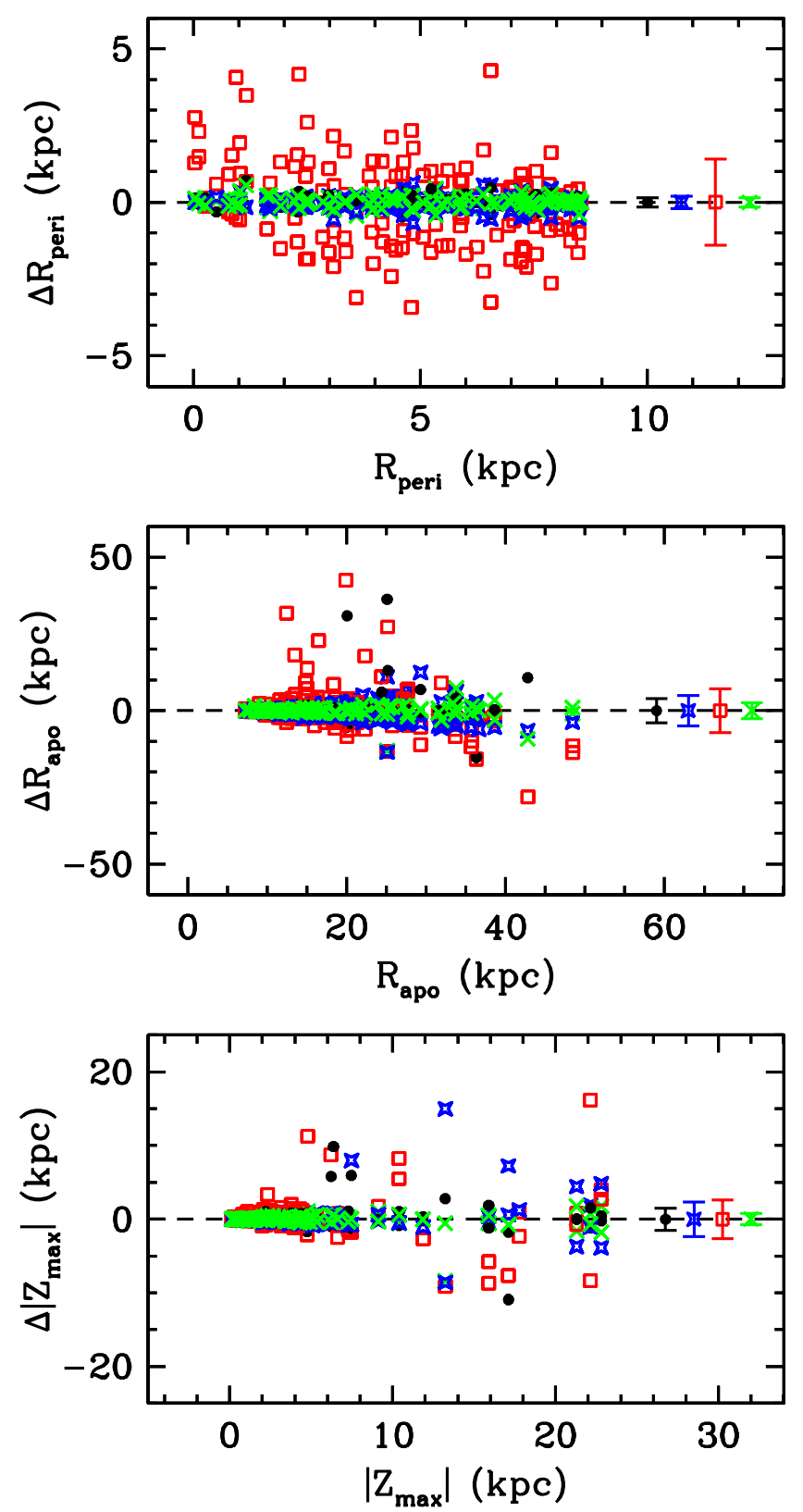

FIG. 4.- Uncertainties in the derived orbital parameters. Changes are shown for alterations in the distance (dark gray circles, black in the online edition), the mass of the Galaxy (open four-point stars, blue in the online edition), the proper motions (open squares, red in the online edition), or the radial velocity (light gray " $\mathrm{X}$ " points, green in the online edition). The $\Delta \mathrm{s}$ refer to (modified parameter)-(unmodified parameter). RMS uncertainties are shown for each set of $\Delta$ values on the right side of each panel. [Please see the electronic edition for a color version of this figure.]

\section{A CAUTION: THE CONNECTION BETWEEN LOW-METALLICITY STARS AND RETROGRADE ORBITS}

Many authors have examined the relationship between $[\mathrm{Fe} / \mathrm{H}]$ and $V_{\phi}$ to characterize the kinematic properties of the thick disk and halo in an effort to better understand the formation mechanisms of these components (e.g., Norris 1986; Sandage \& Fouts 1987; Norris \& Rvan 1989; Beers \& Sommer-Larsen 1995; Chiba \& Yoshii 1998; Chiba \& Beers 2000). Our sample is not designed to rederive the relationship between $[\mathrm{Fe} / \mathrm{H}]$ and $V_{\phi}$, but

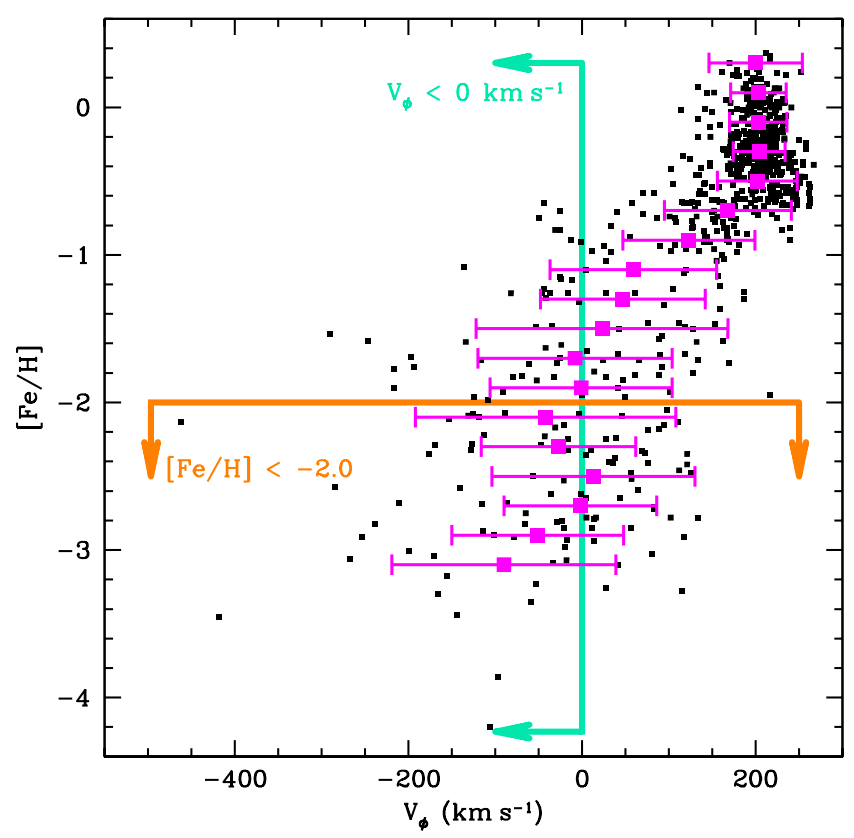

FIG. 5.- Rotational velocity $V_{\phi}$ versus $[\mathrm{Fe} / \mathrm{H}]$ for our sample. Of the $\sim 700$ stars represented on this plot, 94 have retrograde Galactic orbits $\left(V_{\phi}<0 \mathrm{~km} \mathrm{~s}^{-1}\right.$; indicated in turquoise in the online edition) and 93 have $[\mathrm{Fe} / \mathrm{H}]<-2.0$ (indicated in orange in the online edition). A large number of stars (53) fit both categories: $57 \%$ of stars with $[\mathrm{Fe} / \mathrm{H}]<-2.0$ are on retrograde orbits, and $56 \%$ of stars on retrograde orbits have $[\mathrm{Fe} / \mathrm{H}]<-2.0$. The large gray squares (magenta in the online edition) indicate $\left\langle V_{\phi}\right\rangle$ and the associated 1 standard deviation scatter for stars in 0.2 dex bins of $[\mathrm{Fe} / \mathrm{H}]$, but we emphasize that our sample is biased toward metalpoor stars with large space velocities, hence the disproportionately large number of stars with $[\mathrm{Fe} / \mathrm{H}]<-2.0$ and significant retrograde velocities. [Please see the electronic edition for a color version of this figure.]

we must assess the effect of the proper motion biaswhich selects against stars with small proper motionsin our sample before proceeding. Examination of the upper left panel of Figure 3 reveals that many metalpoor stars in our sample have retrograde orbits and vice-versa. We show this more explicitly in Figure 5. Of the 94 stars in our sample on retrograde orbits, 53 $(56 \%)$ also have $[\mathrm{Fe} / \mathrm{H}]<-2.0$. Turning the problem around reveals that, of the 93 stars in our sample with $[\mathrm{Fe} / \mathrm{H}]<-2.0,53(57 \%)$ are on retrograde orbits. This correlation strengthens at lower metallicities: 35 of the 54 stars $(65 \%)$ with $[\mathrm{Fe} / \mathrm{H}]<-2.5$ are on retrograde orbits, and 13 of the 17 stars $(76 \%)$ with $[\mathrm{Fe} / \mathrm{H}]<-3.0$ are on retrograde orbits.

The mean Galactic rotational velocities, $\left\langle V_{\phi}\right\rangle$, binned in 0.2 dex intervals in $[\mathrm{Fe} / \mathrm{H}]$, are shown in Figure 5. We find an approximate relation between $V_{\phi}$ and $[\mathrm{Fe} / \mathrm{H}]$ of $\Delta\left\langle V_{\phi}\right\rangle / \Delta[\mathrm{Fe} / \mathrm{H}] \approx-140 \mathrm{~km} \mathrm{~s}^{-1} \mathrm{dex}^{-1}$ over $-2.0<[\mathrm{Fe} / \mathrm{H}]<-0.6$, lessening to $\Delta\left\langle V_{\phi}\right\rangle / \Delta[\mathrm{Fe} / \mathrm{H}] \approx$ $-90 \mathrm{~km} \mathrm{~s}^{-1} \mathrm{dex}^{-1}$ over $-3.0<[\mathrm{Fe} / \mathrm{H}]<-2.0$. This relationship is a direct consequence of our proper motion bias introduced in $\S$ 2, which would tend to select against very metal-poor stars with near-zero or prograde net rotation, and is not a contradiction to previous results such as Norris (1986), Beers \& Sommer-Larsen (1995), or Chiba \& Beers (2000). The most recent of these studies found $\Delta\left\langle V_{\phi}\right\rangle / \Delta[\mathrm{Fe} / \mathrm{H}] \approx 160 \mathrm{~km} \mathrm{~s}^{-1} \mathrm{dex}^{-1}$ in the range $-1.7<[\mathrm{Fe} / \mathrm{H}]<-0.6$ and a nearly constant $\Delta\left\langle V_{\phi}\right\rangle / \Delta[\mathrm{Fe} / \mathrm{H}] \approx 0 \mathrm{~km} \mathrm{~s}^{-1} \operatorname{dex}^{-1}$ for $-2.6<[\mathrm{Fe} / \mathrm{H}]<$ 
-1.7 in the Solar neighborhood. Presumably, if precise proper motions (even small ones) were known for all the metal-poor stars found in the literature, our relationship between $\left\langle V_{\phi}\right\rangle$ and $[\mathrm{Fe} / \mathrm{H}]$ would flatten out for the same metallicities as found by previous studies.

Some metal-poor stars are identified by high proper motion searches and would be expected to exhibit a kinematic bias; however, a large percentage of metal-poor stars are identified with no kinematic selection criteria (via objective-prism surveys or ultraviolet excess), although these surveys do avoid stars at low Galactic latitude. Also, investigators performing abundance analyses tend to preferentially select the most metal-poor stars for high-resolution followup. Whether because of a selection bias or a true physical preference for extreme orbits (or both), a significant fraction of the stars with $[\mathrm{Fe} / \mathrm{H}] \lesssim-2.0$ that have been subject to high-resolution abundance analyses over the last 15 years have retrograde orbits. It is possible that the large numbers of very metal-poor stars with $[\mathrm{Fe} / \mathrm{H}] \lesssim-3.0$ found in the last few years could reintroduce a slope into the relationship between $\left\langle V_{\phi}\right\rangle$ and $[\mathrm{Fe} / \mathrm{H}]$ at low metallicities, and such a reexamination should be undertaken in the near future.

This relation shown in Figure 5 is important in the context of examining kinematic and chemical correlations in metal-poor stars. Any abundance trend that is preferentially found in low-metallicity stars will also be preferentially found in stars with retrograde velocities, insofar as those velocities are computed based on proper motion measurements with an intrinsic bias. As such, we caution that any abundance trends correlated with retrograde orbits alone should not be over-interpreted as signatures of past accretion events if they can be attributed to nucleosynthetic patterns that inherently occur at low metallicity.

In Figure 6 we show the relationship between metallicity and orbital eccentricity for our inner and outer halo populations, similar to Figure 4 of Eggen et al. (1962). The shaded band in this figure represents the locus of stars used by Eggen et al. (1962). (We convert ultraviolet excess, $\delta(U-B)$, to $[\mathrm{Fe} / \mathrm{H}]$ using the approximate relationships given in Sandage \& Fouts 1987.) Their band encompasses the disk stars at high metallicity and low eccentricity as well as the greater part of our inner halo population, but their sample did not extend to metallicities much lower than $[\mathrm{Fe} / \mathrm{H}]=-2.5$. Many studies since Eggen et al. (1962) have used larger, unbiased datasets to demonstrate that there is no correlation between orbital eccentricity and metallicity (e.g., Chiba \& Beers 2000 and references therein) in the halo. While our inner halo is explicitly chosen to include stars with high eccentricities and our outer halo effectively selects for these stars, too, Figure 6 shows that our entire sample does include metal-poor stars across the full range of eccentricity.

\section{ABUNDANCE RESULTS}

\subsection{Trends with $R_{\text {apo }}$ and $\left|Z_{\max }\right|$}

In Figure 7 we examine the trends of the sodium (Na), magnesium $(\mathrm{Mg})$, calcium $(\mathrm{Ca})$, titanium $(\mathrm{Ti})$, nickel $(\mathrm{Ni})$, yttrium $(\mathrm{Y})$, barium $(\mathrm{Ba})$, and europium $(\mathrm{Eu})$ to Fe ratios as a function of $R_{\text {apo }}$ and $\left|Z_{\max }\right|$ for stars with $[\mathrm{Fe} / \mathrm{H}]<-1.0$. Linear least-squares fits are also shown.

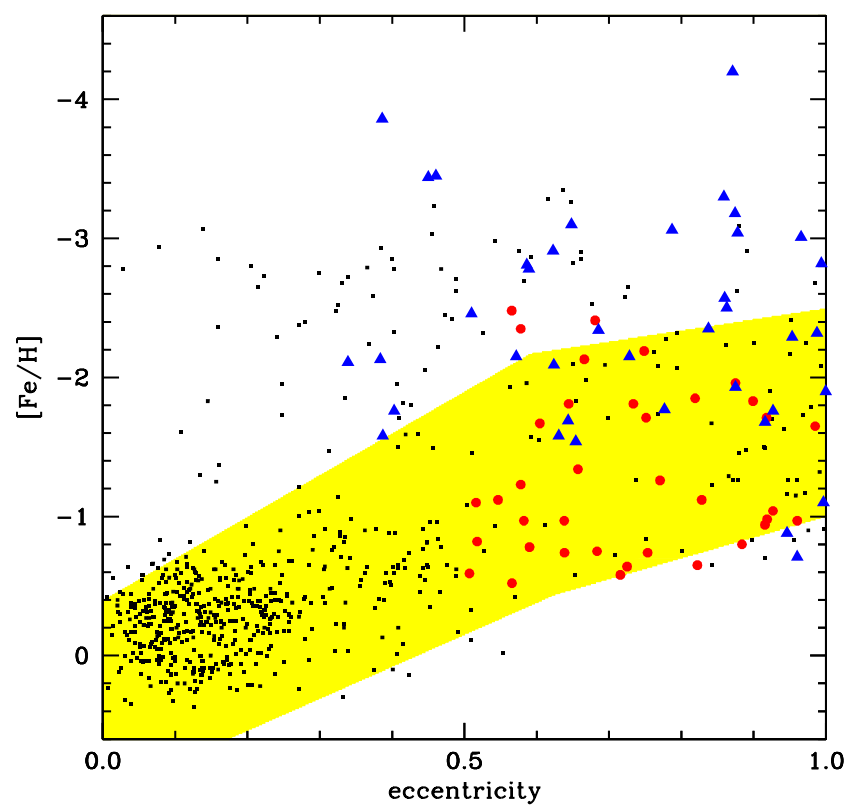

FIG. 6. - The relationship between metallicity and Galactic orbital eccentricity in our sample. Symbols are the same as in Figure 3 The shaded band (yellow in the online edition) represents the relationship identified in Figure 4 of Eggen et al. (1962). [Please see the electronic edition for a color version of this figure.]

No slopes are significant at the $2 \sigma$ level. Using a sample of 11 stars, Stephens (1999) found no trends between $[\alpha / \mathrm{Fe}]$ and $R_{\text {apo }}$ or $\left|Z_{\max }\right|$, a result which we confirm. Using a larger sample (56 stars) and a homogeneous abundance analysis, Stephens \& Boesgaard (2002) found a slight decrease in $\langle[\alpha / \mathrm{Fe}]\rangle\left(-0.0012 \mathrm{dex} \mathrm{kpc}^{-1}\right)$ from $R_{\text {apo }} \sim 8-100$ kpc. Since they used a homogeneous abundance analysis, we defer to their result, but we note that their slope only represents a very subtle change in $[\alpha / \mathrm{Fe}]$ of $\sim 0.1$ dex over $\sim 90 \mathrm{kpc}$. They also found an increase in the cosmic scatter of $[\mathrm{Y} / \mathrm{Fe}]$ at larger $R_{\text {apo }}$. We propose that the complex nucleosynthetic origins of the light neutron-capture species (see $\S$ 8.2) must be better understood before attempting any serious interpretation of these results. Stephens \& Boesgaard (2002) reported no other dependences of abundance ratios with orbital parameters. Combining the Stephens \& Boesgaard (2002) and Fulbright (2000, 2002) datasets, Fulbright (2004) noted slight decreases in all of the light element ratios with respect to $\mathrm{Fe}$ (i.e., $\mathrm{Na}, \mathrm{Mg}, \mathrm{Al}, \mathrm{Si}$ ) and weaker decreases of heavier elements (i.e., Ca, Ti, $\mathrm{Ni}, \mathrm{Y}$, and $\mathrm{Zr}$ ) when using Galactic rest-frame velocity as a surrogate of the model-dependent $R_{\text {apo }}$. Our larger - but inhomogeneous - dataset does not show these trends. The trends reported by Nissen \& Schuster (1997) — whose dataset differed significantly with regard to both kinematics and metallicity range from subsequent studies - have largely not been reproduced by those studies, including ours.

\section{2. $\alpha$ and Iron-Peak Elements in the Inner and Outer Halo Populations}

Figures 8 and 9 display the logarithmic abundance ratios of $\mathrm{Na}, \mathrm{Mg}$, $\mathrm{Ca}$, $\mathrm{Ti}$, and $\mathrm{Ni}$ to $\mathrm{Fe}$ (relative to Solar) for our entire sample and for members of the inner and outer halo populations. For $[\mathrm{Fe} / \mathrm{H}]<-1.0$, both the inner and outer halo populations show super-Solar $[\mathrm{Mg} / \mathrm{Fe}],[\mathrm{Ca} / \mathrm{Fe}]$, and $[\mathrm{Ti} / \mathrm{Fe}] \mathrm{ra}-$ 

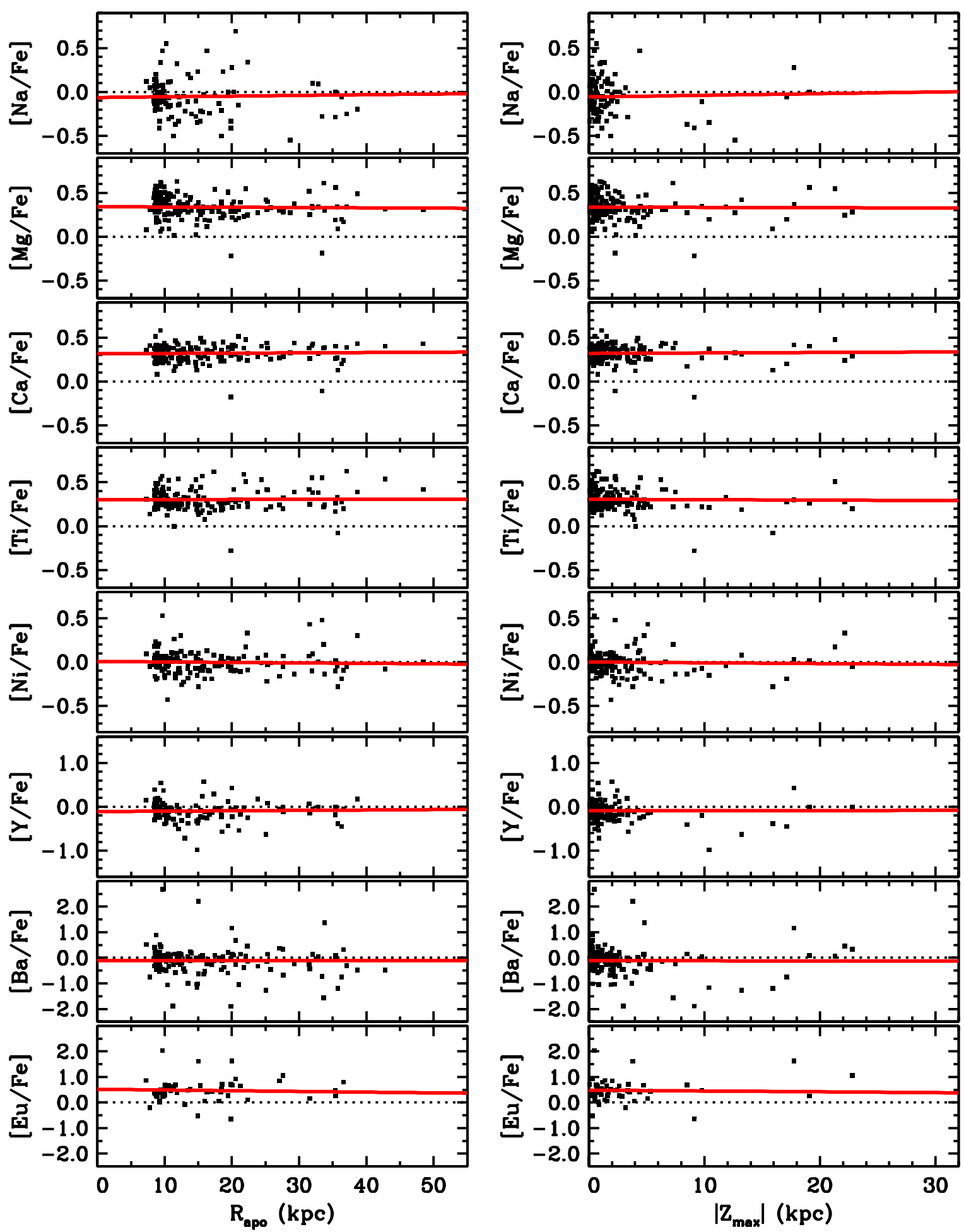

FIG. 7.- Elemental abundance trends as a function of $R_{\text {apo }}$ and $\left|Z_{\max }\right|$ for stars with $[\mathrm{Fe} / \mathrm{H}]<-1.0$. Least squares fits are shown as solid gray lines (red in the online edition). The solar ratios are indicated by the dotted lines. [Please see the electronic edition for a color version of this figure.] 

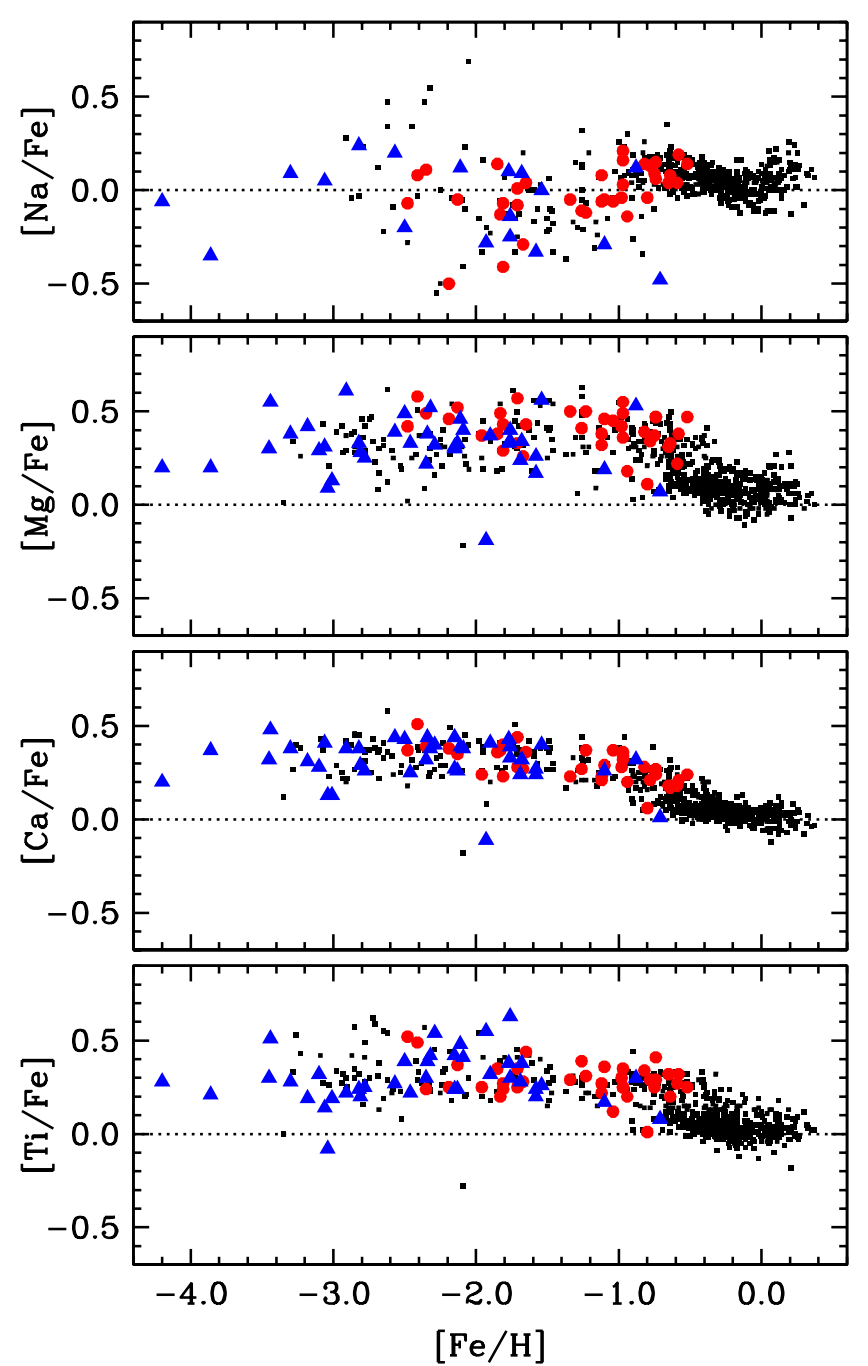

Fig. 8.- $[\mathrm{Na} / \mathrm{Fe}],[\mathrm{Mg} / \mathrm{Fe}],[\mathrm{Ca} / \mathrm{Fe}]$, and $[\mathrm{Ti} / \mathrm{Fe}]$ abundance ratios for our inner (dark gray circles, red in the online edition) and outer (black triangles, blue in the online edition) halo populations. Stars that did not meet the kinematic criteria for these two populations are shown as small dots. [Please see the electronic edition for a color version of this figure.]

tios (hereafter loosely defined as $[\alpha / \mathrm{Fe}]$ ) scattered around +0.3 dex, with no apparent slope below $[\mathrm{Fe} / \mathrm{H}] \lesssim-1.5$. Stephens \& Boesgaard (2002) reported an increase in $[\alpha / \mathrm{Fe}]$ with decreasing $[\mathrm{Fe} / \mathrm{H}]$, a trend not reproduced elsewhere in studies with large numbers of very metalpoor stars (McWilliam et al.|1995b; Carretta et al. 2002; Cavrel et al. 2004; Cohen et al. 2004; Arnone et al. 2005; Lai et al. 2008). The $[\mathrm{Mg} / \mathrm{Fe}]$ scatter may increase at low metallicities, and Stephens \& Boesgaard (2002) only studied 4 stars with $[\mathrm{Fe} / \mathrm{H}]<-3.0$. $[\mathrm{Na} / \mathrm{Fe}]$ exhibits significantly larger scatter (increasing scatter with decreasing metallicity, at least to $[\mathrm{Fe} / \mathrm{H}] \sim-2.3$ ) than any of the $[\alpha / \mathrm{Fe}]$ ratios does, and $[\mathrm{Na} / \mathrm{Fe}]$ approximately follows the Solar ratio. Ni should be produced along with $\mathrm{Fe}$, and $[\mathrm{Ni} / \mathrm{Fe}]$ correlates with $[\mathrm{Fe} / \mathrm{H}]$ at all metallicities with relatively small scatter (increasing also to a maximum below $[\mathrm{Fe} / \mathrm{H}] \lesssim-2.0)$.

One fact is readily apparent from these plots: the bulk of stars in our inner halo sample only are found with metallicities in the range $-2.5 \lesssim[\mathrm{Fe} / \mathrm{H}] \lesssim-0.5$, while most stars in our outer halo are found over the metallicity range $-3.5 \lesssim[\mathrm{Fe} / \mathrm{H}] \lesssim-1.5$. Carollo et al. (2007)

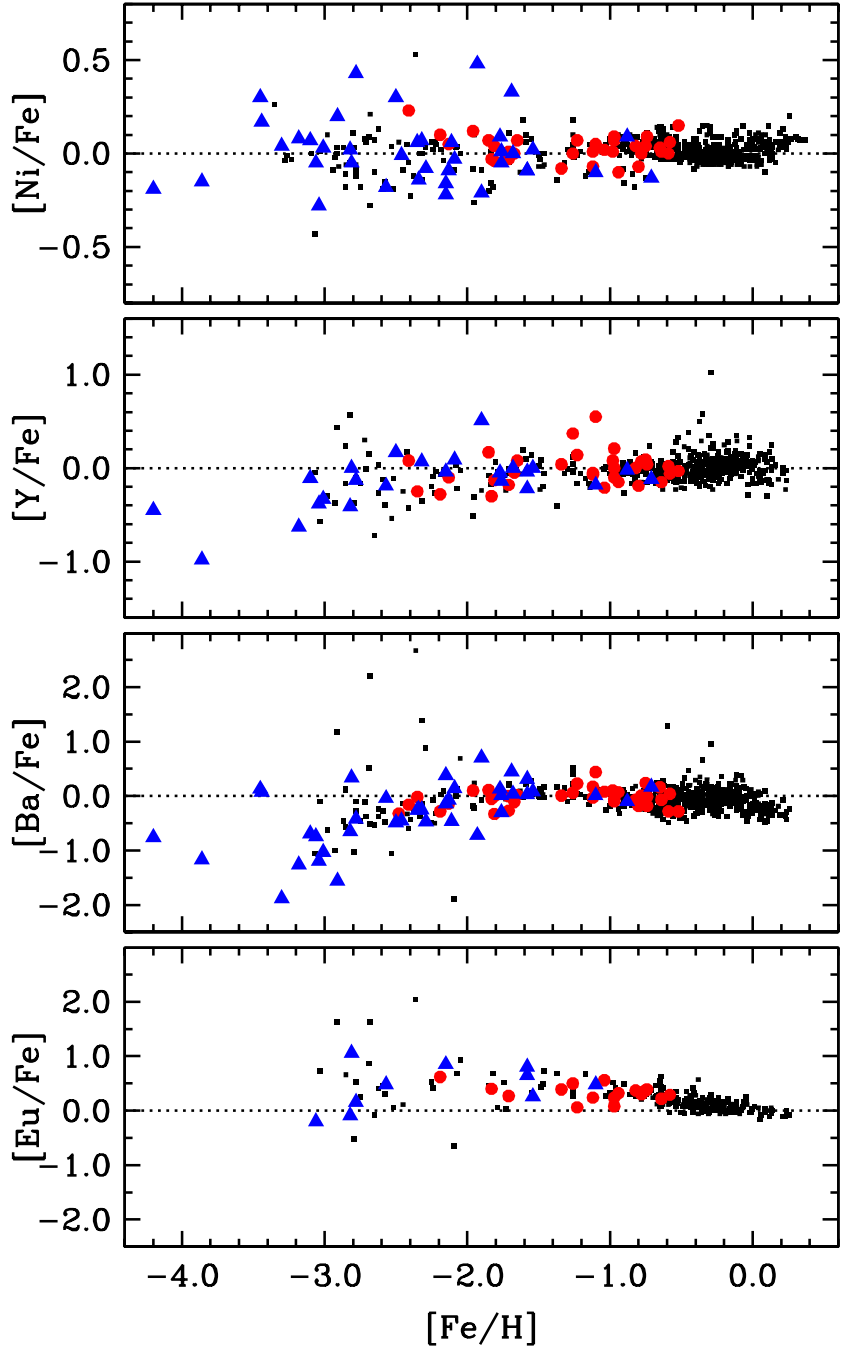

Fig. 9.- $[\mathrm{Ni} / \mathrm{Fe}],[\mathrm{Y} / \mathrm{Fe}],[\mathrm{Ba} / \mathrm{Fe}]$, and $[\mathrm{Eu} / \mathrm{Fe}]$ abundance ratios for our inner and outer halo populations. Symbols are the same as in Figure 8 Upper limits are not displayed. [Please see the electronic edition for a color version of this figure.]

claimed that the metallicity distribution function (MDF) of the inner halo peaks around $[\mathrm{Fe} / \mathrm{H}] \sim-1.6$ and the $\mathrm{MDF}$ of the outer halo peaks around $[\mathrm{Fe} / \mathrm{H}] \sim-2.2$; our results support these assertions. Nevertheless, we caution that (1) our proper motion bias likely selects against lower-metallicity stars on prograde orbits and (2) our sample is drawn from studies designed to select interesting metal-poor stars for detailed abundance analyses, and therefore it should not be used for any assessments of metal-poor MDF's.

Our sample is sensitive to chemical differences in the inner and outer halo populations for stars with the same Fe abundance. Between $-2.3<[\mathrm{Fe} / \mathrm{H}]<-1.6$, the mean $[\mathrm{Mg} / \mathrm{Fe}]$ of the outer halo $\left(\langle[\mathrm{Mg} / \mathrm{Fe}]\rangle=0.30, \sigma_{\text {mean }}=\right.$ $0.05)$ is slightly lower than the mean $[\mathrm{Mg} / \mathrm{Fe}]$ of the inner halo $\left(\langle[\mathrm{Mg} / \mathrm{Fe}]\rangle=0.40, \sigma_{\text {mean }}=0.04\right)$, even when the well-known $\alpha$-poor star G 004-036 $([\mathrm{Fe} / \mathrm{H}]=-1.93$, $R_{\text {apo }}=33_{-4}^{+6} \mathrm{kpc}$, Ivans et al. 2003) is excluded. ${ }^{6}$ This difference is not obvious in $[\mathrm{Ca} / \mathrm{Fe}]$ or $[\mathrm{Ti} / \mathrm{Fe}]$, and the scatter in $[\mathrm{Ca} / \mathrm{Fe}]$ for both populations is noticeably smaller than the scatter in either $[\mathrm{Mg} / \mathrm{Fe}]$ or $[\mathrm{Ti} / \mathrm{Fe}]$. For

6 The quoted uncertainties in the orbital parameters are computed by the same methods discussed in $\S 4.1$ 
all three of $[\mathrm{Mg} / \mathrm{Fe}],[\mathrm{Ca} / \mathrm{Fe}]$, and $[\mathrm{Ti} / \mathrm{Fe}]$, it is worth noting that the "extreme" $[\mathrm{X} / \mathrm{Fe}]$ ratios at a given $[\mathrm{Fe} / \mathrm{H}]$ are predominantly found in the outer halo population.

In Figure 9, this interesting trend begins to emerge more clearly with $[\mathrm{Ni} / \mathrm{Fe}]$ : the inner halo abundance ratios at a given $[\mathrm{Fe} / \mathrm{H}]$ appear much more tightly correlated than the outer halo abundance ratios. The scatter in inner halo $[\mathrm{Ni} / \mathrm{Fe}]$ is commensurate with the typical abundance uncertainties in a given measurement, typically $0.1-0.2$ dex, while the scatter in the outer halo $[\mathrm{Ni} / \mathrm{Fe}]$ is typically $0.5-0.7$ dex (at least for $[\mathrm{Fe} / \mathrm{H}] \lesssim$ $-1.8)$.

In Figure 10 we display the abundance ratios for $[\mathrm{Mg} / \mathrm{Fe}]$ and $[\mathrm{Ni} / \mathrm{Fe}]$ binned as a function of $[\mathrm{Fe} / \mathrm{H}]$. A boxplot is shown for each $[\mathrm{Fe} / \mathrm{H}]$ bin (typically $0.4 \mathrm{dex}$ wide), displaying the median, inner quartiles, and extremes of the entire sample, only the inner halo stars, and only the outer halo stars. For $-2.2 \lesssim[\mathrm{Fe} / \mathrm{H}] \lesssim-1.4$ (where there is significant abundance overlap between the two populations), the median $[\mathrm{Mg} / \mathrm{Fe}]$ ratios for the inner halo are consistently higher than the outer halo medians by $0.10-0.20$ dex. The mean $[\mathrm{Ni} / \mathrm{Fe}]$ ratios of both populations trace the Solar ratio very closely. For $[\mathrm{Ni} / \mathrm{Fe}]$, it is apparent that the extremes of the inner halo are significantly smaller than the extremes of the outer halo. In this case we point to the different degrees of scatter in the inner versus outer populations as evidence for chemical differences between the inner and outer halo populations; no stars with significant deviations from the median ratios are members of the inner halo population. The precise amount of scatter of each population may be affected by the inhomogeneous nature of our sample, but the relative scatter between the two populations is robust.

Our $[\mathrm{Na} / \mathrm{Fe}]$ ratios in Figure 8 exhibit a large degree of scatter. Two of our literature sources for low metallicity stars, Honda et al. (2004a b) and Barklem et al. (2005), did not report $[\mathrm{Na} / \mathrm{Fe}]$ ratios for their samples, thus at low metallicities $([\mathrm{Fe} / \mathrm{H}] \lesssim-2.0)$ there are not enough measurements to adequately compare the inner and outer halo populations. In Figure 11] we show $[\mathrm{Na} / \mathrm{Fe}]$ as a function of $[\mathrm{Mg} / \mathrm{Fe}],[\mathrm{Ca} / \mathrm{Fe}]$, and $[\mathrm{Ti} / \mathrm{Fe}]$. The line in the upper left panel of this figure shows the correlation between $[\mathrm{Mg} / \mathrm{Fe}]$ and $[\mathrm{Na} / \mathrm{Fe}]$ found in field halo giants by Hanson et al. (1998), which generally matches our metal-poor sample. A similar trend (not shown) exists between $[\mathrm{Ca} / \mathrm{Fe}]$ or $[\mathrm{Ti} / \mathrm{Fe}]$ and $[\mathrm{Na} / \mathrm{Fe}]$. Outer halo stars generally occupy the extremes of each distribution, particularly the Na-depleted extremes. This implies that these stars formed from - at least in part - an incompletely mixed ISM where the yields of individual Type II SNe events could still be "noticed" against the overall chemical background of the ISM.

Hanson et al. (1998) reported both an increase in the $[\mathrm{Na} / \mathrm{Fe}]$ scatter with decreasing $[\mathrm{Fe} / \mathrm{H}]$ and an increase in the $[\mathrm{Na} / \mathrm{Fe}]$ scatter when comparing stars on retrograde orbits to those on prograde orbits. To investigate this trend, we turn to a species with more measurements in our dataset, Ni. Previous analyses have revealed correlations between $[\mathrm{Na} / \mathrm{Fe}]$ and $[\mathrm{Ni} / \mathrm{Fe}]$ ratios and stellar kinematic properties (e.g., Nissen \& Schuster 1997, Hanson et al. 1998, Shetrone et al. 2003, Venn et al. 2004). We show this relationship in our sample in Figure 11. The $\mathrm{Na}-\mathrm{Ni}$ relationship originates from the
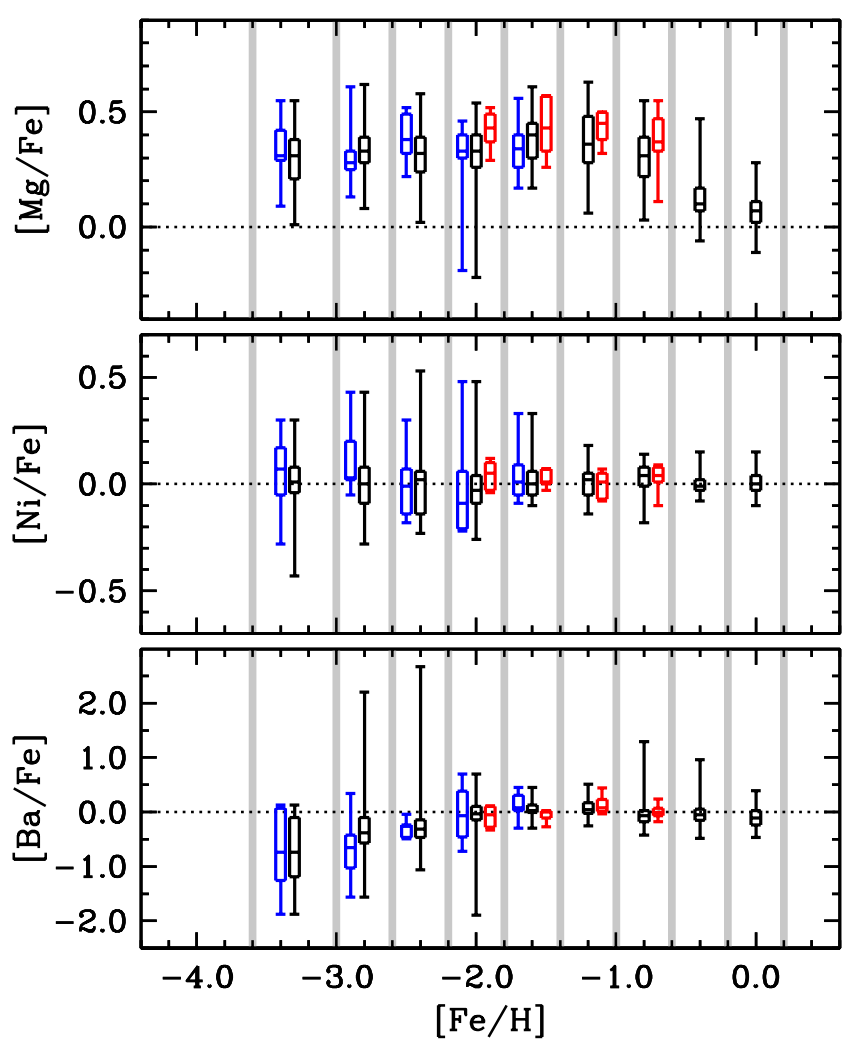

FIG. 10. - Binned abundance ratios for $[\mathrm{Mg} / \mathrm{Fe}],[\mathrm{Ni} / \mathrm{Fe}]$, and $[\mathrm{Ba} / \mathrm{Fe}]$, displayed as quartile boxplots. Black boxes represent all stars in the bin (including the inner and outer halo populations), light gray boxes (red in the online edition) represent the inner halo stars in the bin, and dark gray boxes (blue in the online edition) represent the outer halo stars in the bin. The divisions in the $[\mathrm{Fe} / \mathrm{H}]$ bins are indicated by vertical gray stripes. [Please see the electronic edition for a color version of this figure.]

neutron-rich nature of the dominant isotopes of these species, which can be produced in Type II SNe, albeit in non-Solar ratios. (See Venn et al. 2004 for an extensive discussion of the Na-Ni nucleosynthesis relationship in this context.) This relationship breaks down in material enriched by Type Ia SNe products, since Type Ia SNe produce very little ${ }^{23} \mathrm{Na}$, so we only examine stars with $[\mathrm{Fe} / \mathrm{H}]<-1.0$. Ni-poor stars $([\mathrm{Ni} / \mathrm{Fe}] \leq-0.2)$ are preferentially associated with stars on retrograde orbits; however, Ni-rich stars $([\mathrm{Ni} / \mathrm{Fe}] \geq+0.2)$ and Ni-normal stars $(-0.05 \leq[\mathrm{Ni} / \mathrm{Fe}] \leq+0.05)$ also exhibit a preference for retrograde or no net rotation orbits in our sample. The same effect occurs even if we only consider stars with $[\mathrm{Fe} / \mathrm{H}]<-2.0$, where our proper motion bias is most pronounced. Thus we are unable to confirm or refute the correlation between non-Solar [Ni/Fe] (and, by extension, $\mathrm{Na} / \mathrm{Fe}]$ ) ratios and retrograde orbits.

\subsection{Neutron-Capture Species in the Inner and Outer Halo Populations}

Nuclei heavier than the iron group are formed by the addition of neutrons to existing seed nuclei. The timescales for neutron- $(n-)$ captures determine the resulting abundance patterns. If the average time between successive neutron captures is less than the typical halflife against $\beta^{-}$decay, this is referred to as the slow- $(s-) n$-capture process. In contrast, the rapid- $(r-)$ $n$-capture process occurs when many neutrons are added before any $\beta^{-}$decays can occur. These two processes 

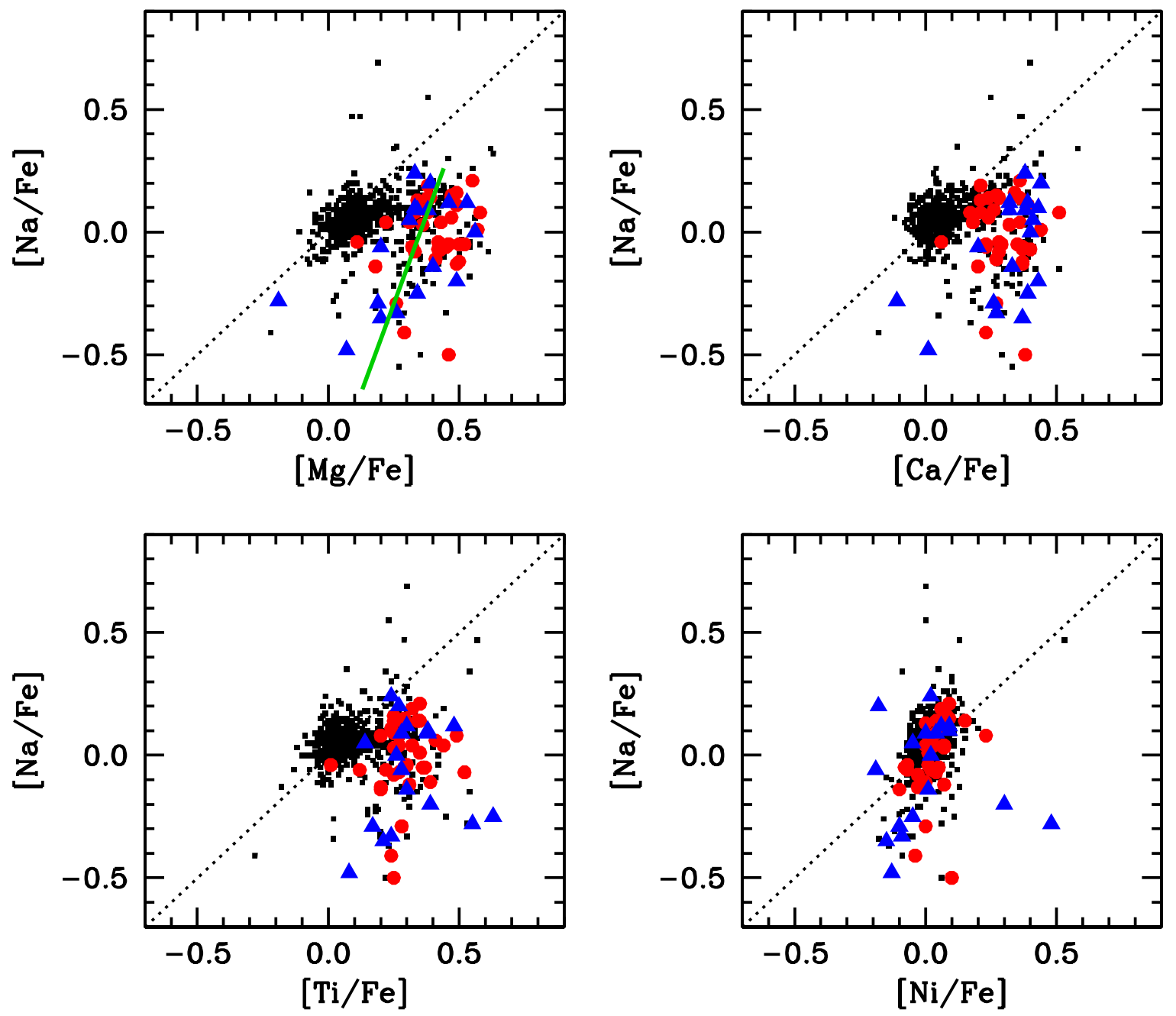

FIG. 11. $[\mathrm{Na} / \mathrm{Fe}]$ as a function of $[\mathrm{Mg} / \mathrm{Fe}],[\mathrm{Ca} / \mathrm{Fe}],[\mathrm{Ti} / \mathrm{Fe}]$, and $[\mathrm{Ni} / \mathrm{Fe}]$ for our inner and outer halo populations. Symbols are the same as in Figure 8$]$ The dotted line represents $[\mathrm{X} / \mathrm{Na}]=+0.0$. The solid line (green in the online edition) in the top left panel is the correlation between $[\mathrm{Mg} / \mathrm{Fe}]$ and $[\mathrm{Na} / \mathrm{Fe}]$ reported by Hanson et al. (1998). [Please see the electronic edition of the journal for a color version of this figure.]

result in very different heavy element abundance patterns. An exact site for the $r$-process has yet to be conclusively identified, but the short stellar timescale necessary to produce $r$-process enrichment in stars with $[\mathrm{Fe} / \mathrm{H}] \lesssim-3.0$ suggests that an association with massive core-collapse Type II SNe is likely. Low and intermediate mass $\left(\sim 1.5-3.0 M_{\odot}\right)$ stars that pass through the AGB phase of evolution are the primary source of $s$-process material (e.g., Busso et al. 1999; Straniero et al. 2006).

In Figure 9 we examine the abundances of the $n$-capture species $[\mathrm{Y} / \mathrm{Fe}],[\mathrm{Ba} / \mathrm{Fe}]$, and $[\mathrm{Eu} / \mathrm{Fe}]$ in our inner and outer halo populations. These ratios display very little scatter for $[\mathrm{Fe} / \mathrm{H}] \gtrsim-1.8$, but they exhibit considerable scatter below $[\widetilde{F e} / \mathrm{H}] \lesssim-1.8$. A familiar trend reappears: the inner halo $[\mathrm{X} / \mathrm{Fe}]$ ratios follow (roughly) a monotonic relationship with $[\mathrm{Fe} / \mathrm{H}]$, while the outer halo $[\mathrm{X} / \mathrm{Fe}]$ ratios scatter about appreciably at a given $[\mathrm{Fe} / \mathrm{H}]$. For example, the scatter in inner halo $[\mathrm{Ba} / \mathrm{Fe}]$ is larger $(\sim 0.5 \mathrm{dex})$ than the typical measurement uncertainty $(\sim 0.1-0.2 \mathrm{dex})$ and follows the Solar ratio, yet the outer halo $[\mathrm{Ba} / \mathrm{Fe}]$ scatter is much larger, typically 1-2 dex (see also, e.g., McWilliam 1998 and Francois et al. 2007). This pattern may also be observed with $[\mathrm{Y} / \mathrm{Fe}]$, though the outer halo scatter is much less extreme. It is more difficult to discern these trends with $[\mathrm{Eu} / \mathrm{Fe}]$, for which fewer measurements exist, and these effects may also be attributed only to increased [Eu/Fe] scatter at the lowest metallicities. The $[\mathrm{Ba} / \mathrm{Fe}]$ ratios, binned by $[\mathrm{Fe} / \mathrm{H}]$, are also shown in Figure [10. The mean $[\mathrm{Ba} / \mathrm{Fe}]$ of all stars traces the Solar value over the range $-2.0 \lesssim[\mathrm{Fe} / \mathrm{H}] \lesssim+0.0$, only declining at the lowest metallicities. The majority of $[\mathrm{Ba} / \mathrm{Fe}]$ ratios in both populations are similar. Stars with extreme ratios only comprise the outer halo; the inner halo ratios are remarkably similar to one another.

The top panel of Figure 12 displays the $[\mathrm{Ba} / \mathrm{Y}]$ ratio as a function of $[\mathrm{Fe} / \mathrm{H}]$ for our inner and outer halo samples. ${ }^{89} \mathrm{Y}(Z=39)$ contains 50 neutrons, which is one of the magic neutron numbers that correspond to closed nuclear shells and significantly lower the nuclear cross section to further neutron capture. During the slow $(s)$ nucleosynthesis reaction, this bottleneck causes lots of nuclei with 50 neutrons to be produced. Thus $\mathrm{Y}$ is representative of the atomic species produced at this first abundance peak in the $s$-process. ${ }^{138} \mathrm{Ba}(Z=56)$ is the dominant isotope of Ba produced in $s$-process nucleosynthesis and also contains a magic number of neutrons, 82 . Ba is representative of the atomic species produced at the second 


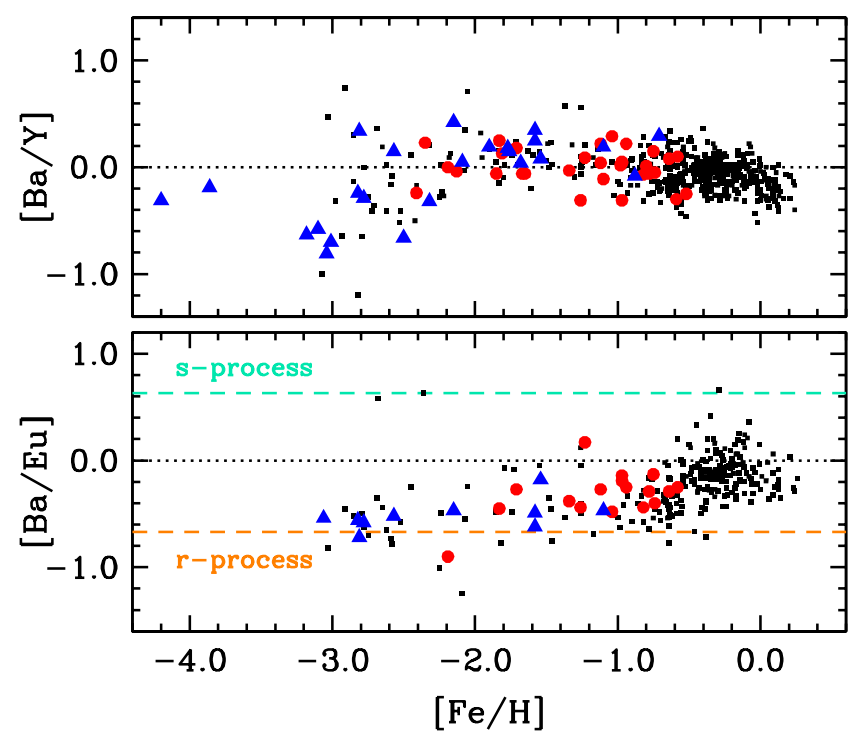

FIG. 12. $-[\mathrm{Ba} / \mathrm{Y}]$ and $[\mathrm{Ba} / \mathrm{Eu}]$ abundance ratios for our inner and outer halo populations. Symbols are the same as in Figure 8 The lower gray dashed line (orange in the online edition) represents the pure $r$-process ratio and the upper gray dashed line (turquoise in the online edition) represents the pure $s$-process ratio as predicted by Simmerer et al. (2004). [Please see the electronic edition for a color version of this figure.]

abundance peak in the $s$-process. The $[\mathrm{Ba} / \mathrm{Y}]$ ratio is useful as a probe of the relative amounts of material produced at these two peaks in the $s$-process. The general decline in $[\mathrm{Ba} / \mathrm{Y}]$ at $[\mathrm{Fe} / \mathrm{H}] \lesssim-2.0$ results from decreasing $\mathrm{Ba}$ contributions from the main $s$-process (see the bottom panel of this figure), while Y production from an apparently primary (i.e., not metallicity-dependent) process remains approximately constant to the lowest metallicities observed (see, e.g., Travaglio et al. 2004). The inner and outer halo members appear to be randomly distributed among the normal scatter for a given $[\mathrm{Fe} / \mathrm{H}]$, and there is no obvious correlation with these populations. (The greatest scatter does appear in the outer halo population at $[\mathrm{Fe} / \mathrm{H}] \lesssim-2.0$ where there is an overall lack of inner halo stars.)

The bottom panel of Figure 12 displays the $[\mathrm{Ba} / \mathrm{Eu}]$ ratio as a function of $[\mathrm{Fe} / \mathrm{H}]$ for our inner and outer halo samples. Relative to $\mathrm{Ba}$, very little $\mathrm{Eu}$ is produced via the $s$-process, yet Eu is relatively easy to produce in the rapid $(r)$ nucleosynthesis reaction, so the $[\mathrm{Ba} / \mathrm{Eu}]$ ratio provides a good assessment of the relative amounts of $s$ - and $r$-process material present in a star. We also show the $[\mathrm{Ba} / \mathrm{Eu}]$ ratios predicted for pure $s$ - or $r$-process nucleosynthesis (Simmerer et al. 2004). (Recall that we have excluded stars with a pure-s-process signature from membership in our inner and outer halo populations.) The stellar data generally decline from the Solar $[\mathrm{Ba} / \mathrm{Eu}]$ ratio at high metallicity toward an $r$-process dominant ratio at low metallicity, though a small number of stars at low-metallicity show evidence of $s$-only enrichment. Again, the inner and outer halo members appear to be randomly distributed among the scatter of all stars at a given $[\mathrm{Fe} / \mathrm{H}]$.

\section{DISCUSSION}

\subsection{The Kinematically and Chemically Diverse Outer} Halo
Is it possible that the inner halo population consistently exhibits a smaller degree of scatter than the outer halo population because of our classification process and not an astrophysical phenomenon? In other words, have we more precisely determined a kinematic population with our "and" selection criterion for the inner halo than with our "or" selection criterion for the outer halo? To address this possibility, we more closely analyze each of our selection criteria for the outer halo population. In Figure 13 we display the $[\mathrm{Mg} / \mathrm{Fe}]$ and $[\mathrm{Ba} / \mathrm{Fe}]$ ratios of stars on increasingly retrograde Galactic orbital velocities, in Figure 14 we display these ratios for stars with increasingly higher values of $\left|Z_{\max }\right|$, and in Figure 15 we display these ratios for stars with increasingly larger values of $R_{\text {apo. }}$. Appreciable increases in the $[\mathrm{Ba} / \mathrm{Fe}]$ scatter are not obvious for stars with the most retrograde velocities, highest distances from the Galactic plane, or increasing maximum distance from the Galactic center. Discernible changes in the $[\mathrm{Mg} / \mathrm{Fe}]$ ratios are not apparent either. Any set of stars sharing one of these outer halo defining characteristics likely would exhibit considerably more abundance scatter than the stars in our inner halo population. The stars in our outer halo population appear to be genuinely kinematically and chemically uncorrelated.

It is somewhat surprising that even a couple of stars in our outer halo population are found at such a high metallicity with "standard" elemental abundance ratios for their metallicity. These stars, HIP 19814 and HIP 117041, with metallicities $[\mathrm{Fe} / \mathrm{H}]=-0.71$ (Stephens \& Boesgaard 2002) and -0.88 (Fulbright 2000), are on orbits extending to $R_{\text {apo }}=27_{-3}^{+4} \mathrm{kpc}$ and $52_{-1}^{+9} \mathrm{kpc}$, respectively. These stars are also on highly eccentric orbits ( $e=0.96$ and $e=0.95$, respectively) and may be in the metal-rich end of the (outer) halo MDF. The uncertainty in $R_{\text {apo }}$ for HIP 19814 could marginally demote this star from outer halo membership; even so, its Galactic orbit would remain eccentric.

It has long been common practice to assume that ancient metal-poor stars do not accrete any appreciable amount of metals (certainly not enough to enrich a metalfree star to $[\mathrm{Fe} / \mathrm{H}] \sim-4.0)$ from passage through the gasrich Galactic disk (see commentary on this subject by, e.g., Yoshii 1981, Iben 1983, and Frebel et al. 2008b.). If this is so, then the composition and Galactic orbits of these stars suggest that significant metal enrichment $(\sim 1 / 5$ to $1 / 8$ Solar $\mathrm{Fe})$ may have occurred in some localized regions far from the present Galactic disk. This is in qualitative agreement (but perhaps not quantitative, since our local sample of stars may not be representative of the bulk of the stellar halo; see $\S 80$ with the halo chemical evolution model presented by Tumlinson (2006), who found that some stars in the metal-rich end of the halo MDF were forming within the first few hundred million years after star formation began.

A wide diversity of stellar orbits and chemical compositions is found in our outer halo population, which is strong evidence that a significant fraction of the halo was formed from the conglomeration of small fragments representing a variety of nucleosynthetic enrichment scenarios (e.g., Searle \& Zinn 1978). 

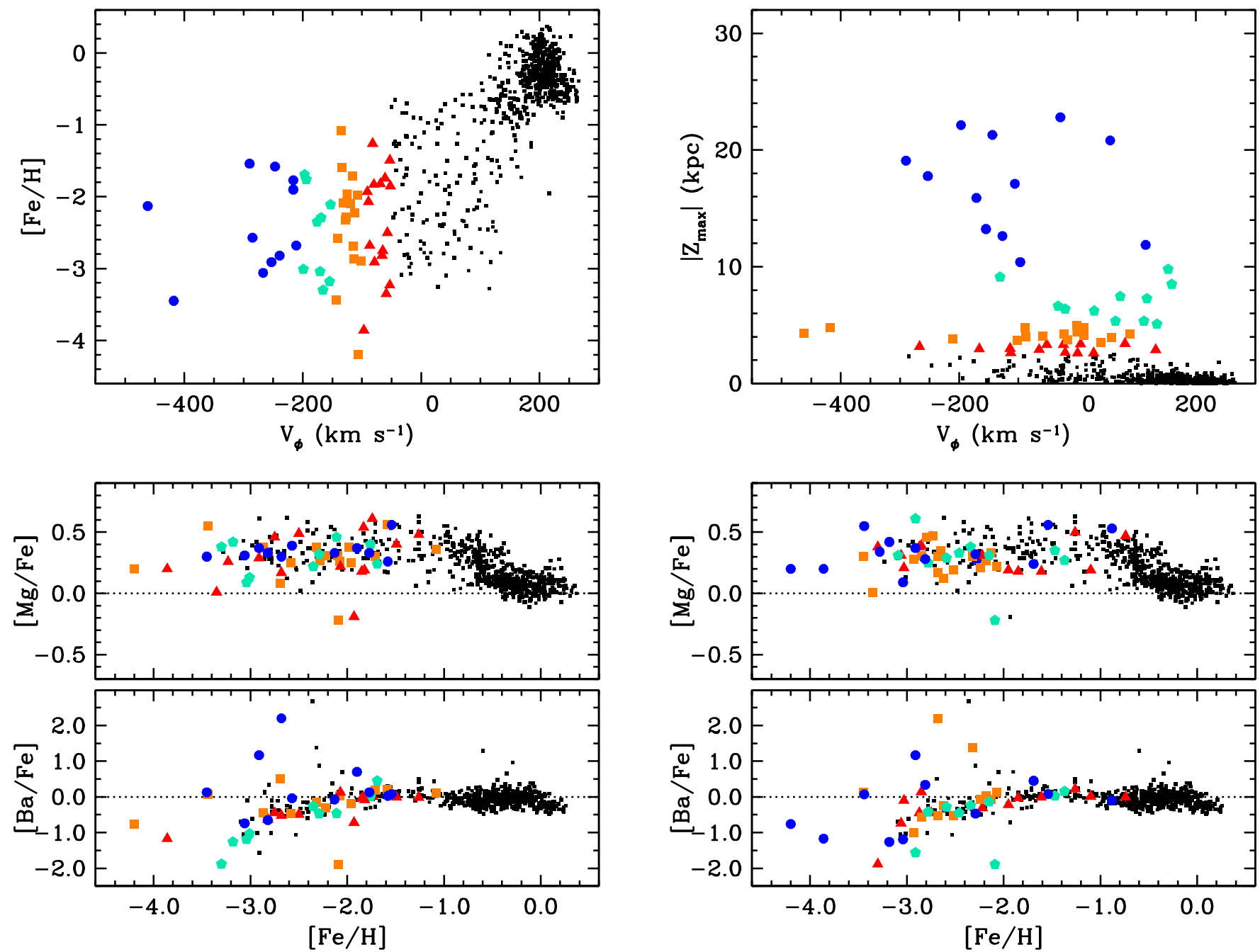

FIG. 13. - Identifying stars of increasing retrograde rotation about the Galaxy and associated $[\mathrm{Mg} / \mathrm{Fe}]$ and $[\mathrm{Ba} / \mathrm{Fe}]$ abundance ratios. Stars are selected based only on their rotation velocity: $-100<V_{\phi} \leq-50 \mathrm{~km} \mathrm{~s}^{-1}$ (filled gray triangles, red in the online edition), $-150<V_{\phi} \leq-100$ (filled gray squares, orange in the online edition), $-200<V_{\phi} \leq-150$ (filled gray pentagons, turquoise in the online edition), and $V_{\phi} \leq-200$ (filled gray circles, blue in the online edition). The stars selected according to these definitions are highlighted in the lower two panels. [Please see the electronic edition for a color version of this figure.]

\subsection{Relationship to the Inner and Outer Halo Globular Clusters}

Globular clusters can be classified according to their Galactic orbital parameters, traditionally defined such that "outer halo" clusters have orbits that take them to much greater radii from the Galactic center than "inner halo" clusters. Might we learn any additional information by classifying clusters according to the inner and outer halo population definitions given in $\S$ 4? We use a sample of 25 globular clusters with measured distances and space velocities compiled from the literature by Pritzl et al. (2005), who derived mean abundance ratios for each cluster from recent high-resolution spectroscopic analyses of individual stars in each cluster. The cluster positions are taken from the most recent version of the Harris (1996) catalog (February 2003), and the cluster velocities are taken from a series of papers by Dinescu et al. (1999, 2000, 2001, 2003). We compute or-

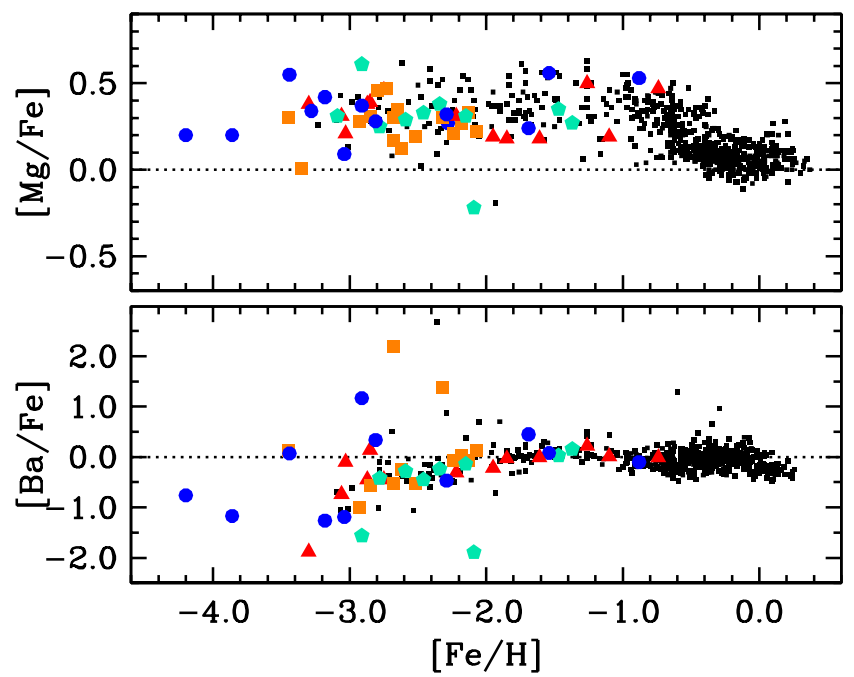

FIG. 14.- Identifying stars of increasing vertical distance from the Galactic plane and associated $[\mathrm{Mg} / \mathrm{Fe}]$ and $[\mathrm{Ba} / \mathrm{Fe}]$ abundance ratios. Stars are selected based only on $\left|Z_{\max }\right|: 2.5<$ $\left|Z_{\max }\right| \leq 3.5 \mathrm{kpc}$ (filled gray triangles, red in the online edition), $3.5<\left|Z_{\max }\right| \leq 5$ (filled gray squares, orange in the online edition), $5<\left|Z_{\max }\right| \leq 10$ (filled gray pentagons, turquoise in the online edition), and $\left|Z_{\max }\right|>10$ (filled gray circles, blue in the online edition). The stars selected according to these definitions are highlighted in the lower two panels. [Please see the electronic edition for a color version of this figure.]

bital parameters using our model for the Galactic potential. Table 4 displays the adopted distances and velocities and our derived orbital parameters for this set of clusters. Four clusters match our inner halo kinematic criteria (M4, M71, NGC 6397, and NGC 6752), ranging in metallicity from $-2.0 \leq[\mathrm{Fe} / \mathrm{H}] \leq-0.7$. Eight clusters match our outer halo kinematic criteria (M3, M30, M68, NGC 288, NGC 362, NGC 5466, Pal 5, and Pal 12), ranging in metallicity from $-2.4 \leq[\mathrm{Fe} / \mathrm{H}]-0.7$. Pal 12 has been conclusively identified as having been stripped from the Sagittarius dSph; excluding Pal 12, our outer halo globular clusters span the metallicity range $-2.4 \leq[\mathrm{Fe} / \mathrm{H}]-1.3$.

In Figure 16 we compare the $[\mathrm{Mg} / \mathrm{Fe}],[\mathrm{Ca} / \mathrm{Fe}]$, and $[\mathrm{Ti} / \mathrm{Fe}]$ abundance ratios for our inner and outer halo globular clusters and our inner and outer halo field stars. In all three cases, the abundance ratios of the inner halo globular clusters obey the same trends and degree of scatter defined by the inner halo field stars. The outer halo 

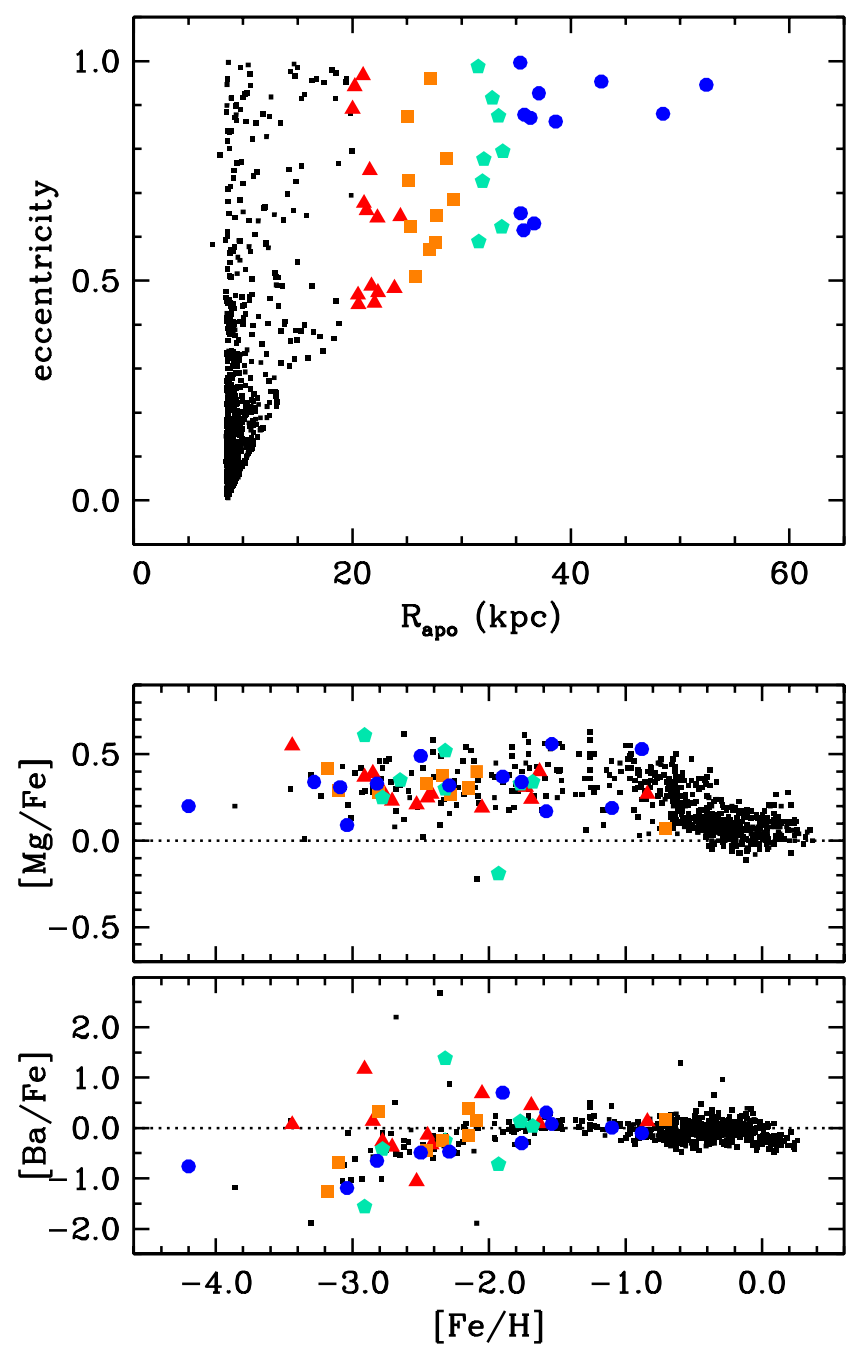

FIG. 15.- Identifying stars of increasing maximum radial distance from the Galactic center and associated $[\mathrm{Mg} / \mathrm{Fe}]$ and $[\mathrm{Ba} / \mathrm{Fe}]$ abundance ratios. Stars are selected based only on $R_{\text {apo: }}$ $20<R_{\text {apo }} \leq 25 \mathrm{kpc}$ (filled gray triangles, red in the online edition), $25<R$ apo $\leq 30$ (filled gray squares, orange in the online edition), $30<R_{\text {apo }}<35$ (filled gray pentagons, turquoise in the online edition), and $R_{\text {apo }}>35$ (filled gray circles, blue in the online edition). The stars selected according to these definitions are highlighted in the lower two panels. [Please see the electronic edition for a color version of this figure.]

globular clusters likewise follow the trends and scatter of the outer halo field stars, with a few exceptions. $[\mathrm{Mg} / \mathrm{Fe}]$ and $[\mathrm{Ca} / \mathrm{Fe}]$ are marginally low (but still super-Solar) in NGC 5466, though they have been derived from a single Cepheid variable (McCarthy \& Nemec 1997) and should be treated with some caution; even so, NGC 5466 is within the scatter expected for an outer halo cluster. $[\mathrm{Ti} / \mathrm{Fe}]$ is Solar in M68, which helped lead Lee et al. (2005) to postulate that M68 may have sampled an IMF biased toward higher masses, where the $\mathrm{Mg}$ and $\mathrm{Si}$ overabundances were produced by Type II SNe. In this scenario $\mathrm{Ti}$ would be primarily produced by by lower mass SN Ia along with the Fe-peak elements. (See also the extensive discussion in Lee \& Carney 2002, who identified constant $[\mathrm{Ca} / \mathrm{Fe}]$ but decreasing $[\mathrm{Si} / \mathrm{Fe}]$ and increasing $[\mathrm{Ti} / \mathrm{Fe}]$ ratios with current Galactocentric radius in their sample, and Gratton et al. 2004, who also reproduced this result when using $R_{\text {apo. }}$ ) Finally, M68 does not appear to be associated with the Canis Ma-

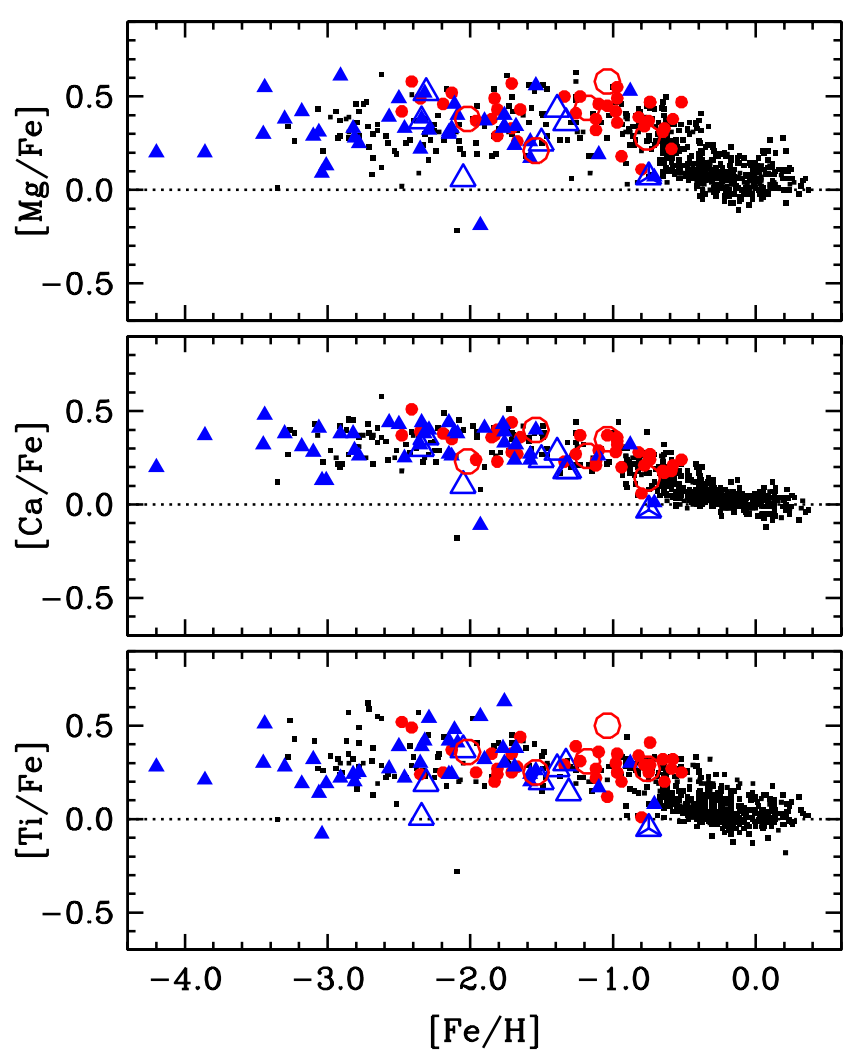

FIG. 16. - Mean $[\mathrm{Mg} / \mathrm{Fe}],[\mathrm{Ca} / \mathrm{Fe}]$, and $[\mathrm{Ti} / \mathrm{Fe}]$ abundance ratios for globular clusters with kinematics like those that define our inner and outer halo (field star) populations. Globular clusters with kinematics like the inner halo are indicated by the large, open gray circles (red in the online edition), and globular clusters with kinematics like the outer halo are indicated by the large, open black triangles (blue in the online edition). The triangle marked with an "X" indicates Pal 12, which has been conclusively identified as a cluster accreted from the Sagittarius dSph. All other symbols are the same as in Figure 8 The Solar ratios are indicated by the dotted lines. [Please see the electronic edition for a color version of this figure.]

jor dSph (Pritzl et al. 2005), as had been suggested by earlier models (Martin et al. 2004); however, Pritzl et al. (2005) have suggested that it may have an extragalactic origin based on its younger age, high $[\mathrm{Si} / \mathrm{Ti}]$ ratio, and high prograde rotational velocity.

Figure 17 displays the $[\mathrm{Y} / \mathrm{Fe}],[\mathrm{Ba} / \mathrm{Fe}]$, and $[\mathrm{Eu} / \mathrm{Fe}]$ ratios for our inner and outer halo field stars and globular clusters. The inner and outer halo globular clusters possess $[\mathrm{Ba} / \mathrm{Fe}]$ and $[\mathrm{Eu} / \mathrm{Fe}]$ ratios that very closely follow these ratios in the inner halo field stars with very small scatter. To some degree, this reflects the fact that we have represented the abundance ratios by means rather than the scatter intrinsic from one star to another within a given cluster; however, this scatter is much smaller (typically $\lesssim 0.5 \mathrm{dex}$ ) than that found for field stars $(\geq 2$ dex $)$, so it cannot tell the full story (cf., e.g., Sneden et al. 1997, 2000, Ivans et al. 2001, Gratton et al. 2004, Yong et al. 2008). This theme -also present in recent reviews of globular cluster abundances (Gratton et al. 2004; Sneden et al. 2004) - suggests that these globular cluster stars formed from a homogenized ISM much like the field stars of the inner halo. In this sense, the abundance trends traced by our inner halo population and the globular cluster population may represent a time-averaged set of chemical yields for a metal- 


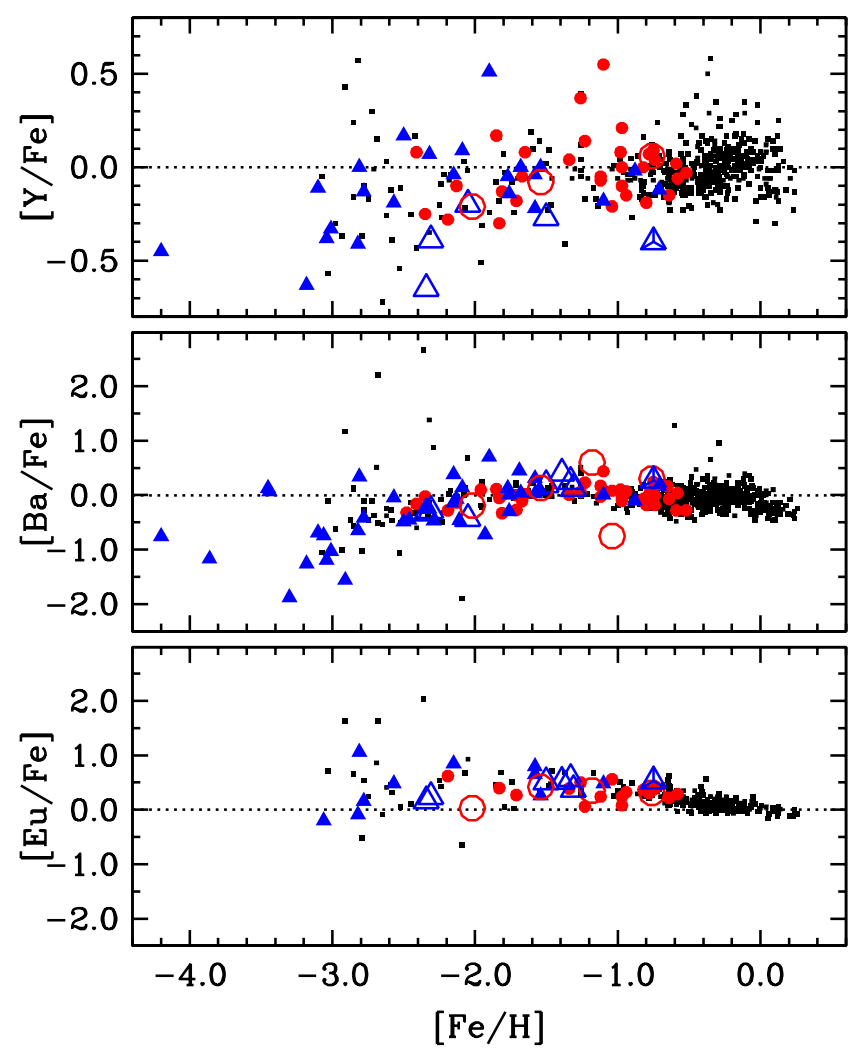

Fig. 17.- Mean [Y/Fe], $[\mathrm{Ba} / \mathrm{Fe}]$, and $[\mathrm{Eu} / \mathrm{Fe}]$ abundance ratios for globular clusters with kinematics like those that define our inner and outer halo populations. Symbols are the same as in Figure 16 [Please see the electronic edition for a color version of this figure.]

poor stellar population. If the earliest generations of stars pre-enriched the ISM from which the present stars formed, this would also explain the lack of stars with $[\mathrm{Fe} / \mathrm{H}] \lesssim-2.5$ in the inner halo and globular clusters.

The $[\mathrm{Y} / \mathrm{Fe}]$ ratios implore us to exercise some caution with this interpretation. Mean $[\mathrm{Y} / \mathrm{Fe}]$ ratios for the five outer halo clusters with kinematic and abundance data are consistently lower than the mean $[\mathrm{Y} / \mathrm{Fe}]$ ratios for the three inner halo clusters $\left(\langle[\mathrm{Y} / \mathrm{Fe}]\rangle_{\text {outer }}=-0.38\right.$, $\left.\sigma_{\text {mean }}=0.13 ;\langle[\mathrm{Y} / \mathrm{Fe}]\rangle_{\text {inner }}=-0.08, \sigma_{\text {mean }}=0.11\right)$ These differences are less apparent in Figure [18, where we display the $[\mathrm{Ba} / \mathrm{Y}]$ and $[\mathrm{Ba} / \mathrm{Eu}]$ ratios, yet four of the five outer halo clusters do have super-Solar $[\mathrm{Ba} / \mathrm{Y}]$ ratios. This might suggest a decreased contribution from very massive SNe (see $\S 8.2$ ), although analysis of a more homogeneous dataset including abundances for more clusters with kinematic information would be necessary to draw any robust conclusions.

Finally, in Figure [19 we plot the $[\mathrm{Ca} / \mathrm{Fe}],[\mathrm{Ti} / \mathrm{Fe}]$, $[\mathrm{Y} / \mathrm{Fe}]$, and $[\mathrm{Ba} / \mathrm{Fe}]$ ratios as a function of $R_{\text {apo }},\left|Z_{\max }\right|$, and $V_{\phi}$. Excluding Pal $12,[\mathrm{Ca} / \mathrm{Fe}]$ exhibits no trends with these kinematic properties, while earlier reports of a [Ti/Fe] trend with $R_{\text {apo }}$ appear less secure with this dataset. No obvious trends of $[\mathrm{Ba} / \mathrm{Fe}]$ with kinematics are visible. Only $\mathrm{Y}$-deficient $([\mathrm{Y} / \mathrm{Fe}]<-0.2)$ clusters are found at $R_{\text {apo }}>10 \mathrm{kpc}$ or $\left|Z_{\max }\right|>5 \mathrm{kpc}$, including Pal 12. Pal 12 displays the low $[\alpha / \mathrm{Fe}]$ ratios and extreme kinematics associated with being a captured cluster. $^{7}$ Could these properties be used to diagnose ad-

7 Pal 12 has low $\mathrm{Mg}$, Ca, and Ti, which closely correspond to the notoriously low $\mathrm{Mg}, \mathrm{Ca}$, and Ti abundances of the Sagittarius dSph

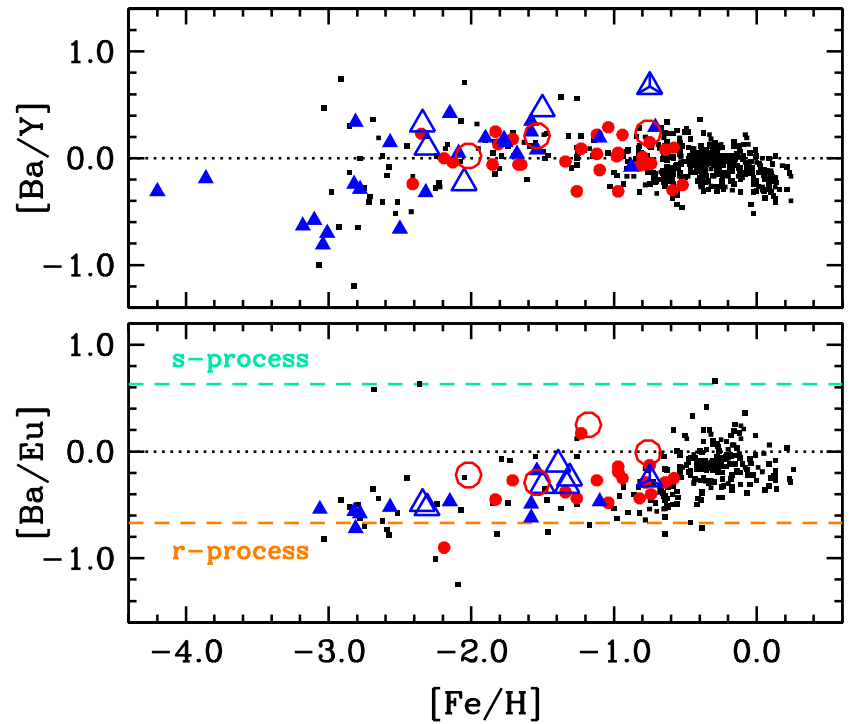

FIG. 18. - Mean $[\mathrm{Ba} / \mathrm{Y}]$ and $[\mathrm{Ba} / \mathrm{Eu}]$ abundance ratios for globular clusters with kinematics like those that define our inner and outer halo populations. Symbols are the same as in Figure 16 [Please see the electronic edition for a color version of this figure.]

ditional clusters that have been captured from dSphs? Given the inhomogeneous abundance analysis methods, wide range of number of stars examined in each cluster, abundance dispersions within clusters, and range of mean elemental abundance ratios for clusters already examined, we suspect that these diagnostics may be more useful for confirming capture proposals based on kinematic evidence rather than as a priori criteria (such as the case made for Pal 12 by Cohen 2004).

\subsection{Is the Inner Halo a Remnant of an Accreted Dwarf Galaxy?}

The orbital characteristics we have used to identify members of the inner halo population are reminiscent of a clump of stars found with $e \sim 0.9,[\mathrm{Fe} / \mathrm{H}] \sim-1.7$, and near-zero (or perhaps just slightly prograde) net rotation by Chiba \& Beers (2000). They speculated that a significant fraction of the stars in this clump may have formed from infalling gas with this metallicity. The very small abundance scatter for other species at a given $[\mathrm{Fe} / \mathrm{H}]$ in our inner halo population supports this interpretation. Dinescu (2002) articulated that a slight retrograde clump of stars spanning $-2.0<[\mathrm{Fe} / \mathrm{H}]-1.5$ in the Beers et al. (2000) data (employed also by Chiba \& Beers 2000) could be reconciled with stellar debris associated with $\omega$ Centauri, the stripped core of an accreted dSph (e.g., Norris et al. 1997; Dinescu et al. 1999) that has a similar peak in its MDF (Norris et al. 1996). Could our inner halo population share a similar origin with this $\omega$ Cen debris (which could also help to place age constraints on the inner halo)? Comparing the chemical abundances of $\omega$ Cen giants (Norris \& Da Costa 1995) with our inner halo sample reveals notable differences in $\mathrm{Ca}, \mathrm{Ti}, \mathrm{Ni}$, $\mathrm{Y}$, and $\mathrm{Ba}$ trends with metallicity, effectively dismissing this hypothesis. Furthermore, it is unlikely that a sizable fraction of the entire stellar halo of the Milky Way

and its associated globular cluster system (Bonifacio \& Caffau 2003; Tautvaišienė et al. 2004; Cohen 2004; Sbordone et al. 2007), although the low-metallicity globular clusters associated with the Sagittarius dSph have elevated $[\alpha / \mathrm{Fe}]$ ratios (Mottini et al. 2008). 


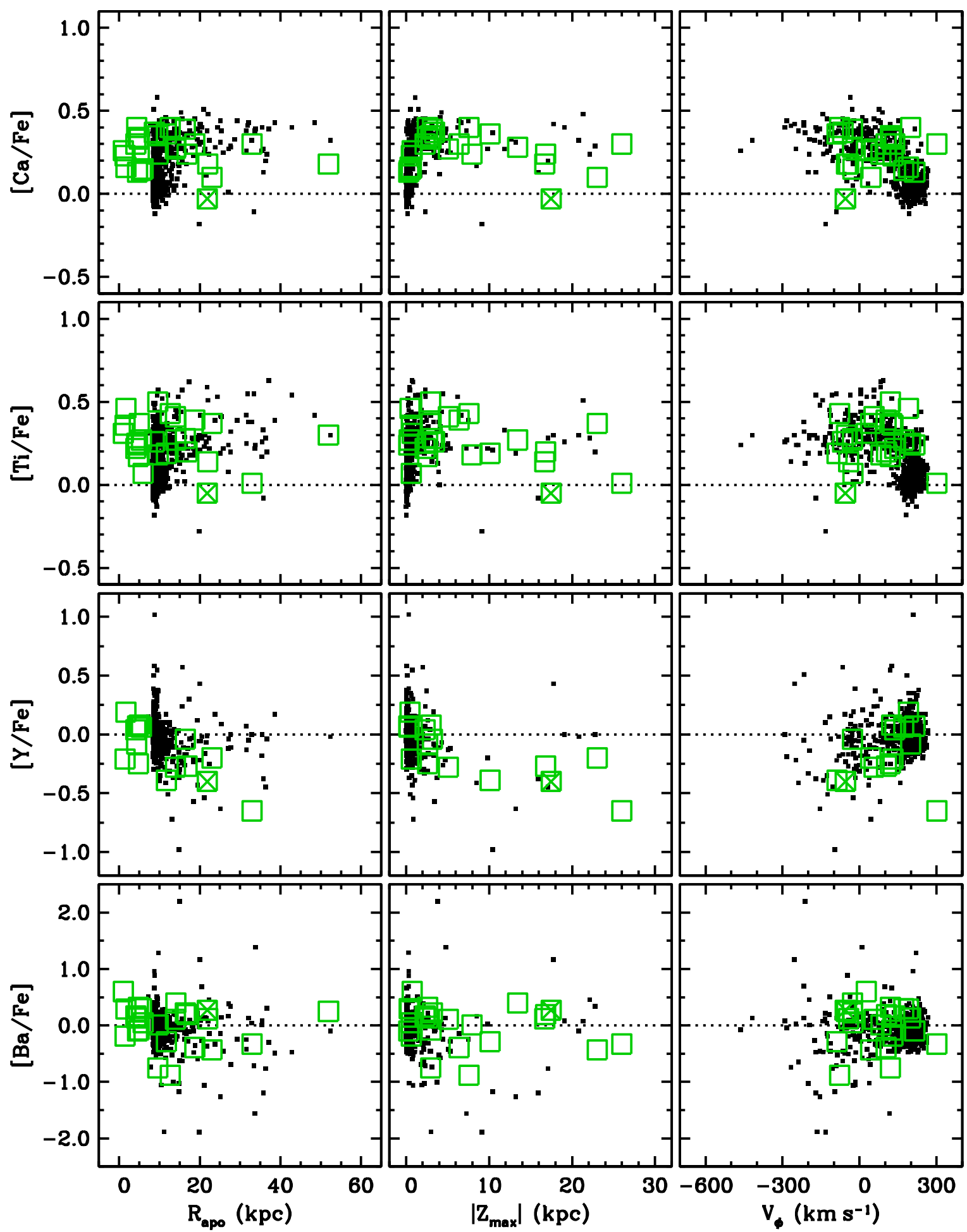

FIG. 19.- $[\mathrm{Ca} / \mathrm{Fe}],[\mathrm{Ti} / \mathrm{Fe}],[\mathrm{Y} / \mathrm{Fe}]$, and $[\mathrm{Ba} / \mathrm{Fe}]$ ratios for globular clusters as a function of $R_{\mathrm{apo}},\left|Z_{\max }\right|$, and $V_{\phi}$. Globular clusters are indicated by the large, open black squares (green in the online edition) and our stellar sample is indicated by the small gray dots. Pal 12 is indicated by the square marked with an "X". The Solar ratios are indicated by the dotted lines. [Please see the electronic edition for a color version of this figure.] 


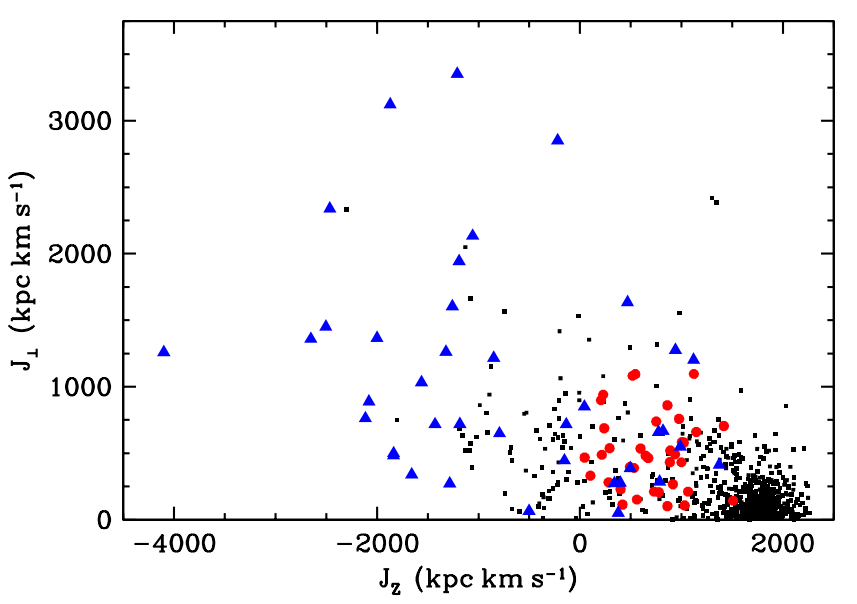

FIG. 20.- Inner and outer halo members as a function of their angular momentum components. $J_{\perp}$ is defined as $\left(J_{X}^{2}+J_{Y}^{2}\right)^{1 / 2}$. Symbols are the same as in Figure 3 [Please see the electronic edition for a color version of this figure.]

Galaxy (which we associate with the inner halo population) was composed of stars accreted from a single dSph.

The inner halo abundances might still suggest that this population resembles a large-scale stellar stream (e.g., Kepley et al. 2007; Helmi 2008, and references therein). While the spatial density distribution of stellar streams has long since dissipated, their velocity space density remains more intact (Helmi \& White 1999). In Figure 20 we show our inner and outer halo populations expressed in terms of their angular momenta $J_{X}=y W-V z$, $J_{Y}=z U-W x, J_{Z}=x V-U y$, and $J_{\perp}=\left(J_{X}^{2}+J_{Y}^{2}\right)^{1 / 2}$ (per unit mass, where $[x, y, z]$ and $[U, V, W]$ are the positions and velocities, respectively, of a star in a lefthanded Galactocentric frame). The components of the angular momentum for each star are also listed in Table 1. The outer halo stars are preferentially found (as per our selection criteria) at low values of $J_{Z}$ and high values of $J_{\perp}$. In contrast to the outer halo, the inner halo stars occupy a limited region of the diagram, and this region appears as part of the continuous progression from stars on disk-like orbits to stars on orbits that take them far from the Galactic center. In this regard the inner halo certainly looks like a more coherent kinematic component of the halo (even if it is too large to be realistically classified as a stellar "stream") than the outer halo, which looks like a smattering of stars on completely unrelated orbits.

In light of these arguments, it is perhaps even more remarkable that, of the eight $[\mathrm{X} / \mathrm{Fe}]$ ratios considered for our halo populations in the previous sections, not a single abundance ratio of any inner halo star deviates even minimally from the well-defined mean abundance trends of the inner halo population (though $[\mathrm{Na} / \mathrm{Fe}]$ shows an overall greater amount of scatter). Even the heavy $n$-capture species obey a tight correlation with $[\mathrm{Fe} / \mathrm{H}]$, an abundance pattern rarely seen at $[\mathrm{Fe} / \mathrm{H}] \lesssim-2.0$, even in dSphs (e.g., Shetrone et al. 2003, A. Frebel et al., in prep.). This is strong evidence for one of two possibilities. Either the stars in our inner halo population formed from a very homogenized ISM, or the time-averaged abundance yields of various regions of the stellar halo are nearly identical. Both scenarios could also explain the apparent lack of very low metallicity stars in the inner halo population, since it may have been common prac- tice for stars with $[\mathrm{Fe} / \mathrm{H}] \ll-2.0$ to form in regions of the halo where chemical enrichment was still governed by local SNe events, rather than the time-averaged yields of many SNe (e.g., Argast et al. 2000).

\subsection{Chemical Signatures of Possible Accretion Events \\ 7.4.1. Blue Metal-Poor Stars}

Blue metal-poor (BMP) stars are the field analogs of the blue straggler stars found in clusters, except BMP's are believed to be formed by binary mass-transfer rather than stellar mergers (Preston \& Sneden 2000; Sneden et al. 2003b; Carney et al. 2005). Preston et al. (1994) have suggested that BMP stars may signify accretion events. Based on new binary orbital solutions and analysis of their chemical compositions, however, Preston \& Sneden (2000) and Sneden et al. (2003b) suggested that only the radial velocity $(\mathrm{RV})$ constant BMP stars may be intermediate-age stars that have been accreted, perhaps from satellite dSph systems.

Of the 175 stars examined by Preston et al. (1994), two are present in our sample, CS 22966-043 and CS 22941-012. CS 22966-043, a long period binary with a nearly circular binary orbit $(P=317$ days, $e=0.1$; Preston \& Sneden 2000), possesses a heretofore unique mix of the $\alpha$ elements for a star with $[\mathrm{Fe} / \mathrm{H}]=-1.91$ : $[\mathrm{Mg} / \mathrm{Fe}]=-0.65,[\mathrm{Ca} / \mathrm{Fe}]=-0.24$, and $[\mathrm{Ti}$ II $/ \mathrm{Fe}]=$ +0.49 (Ivans et al. 2003). CS 22941-012, a RV-constant star (Preston \& Sneden 2000), was employed as a BMP, $\alpha$-normal comparison to CS $22966-043$ by Ivans et al. (2003). CS 22966-043 barely did not pass our proper motion requirement. If we relax this requirement and naively adopt a photometric distance, CS 22966-043 is on a very extreme orbit, only circling the Galactic center 4-8 times over $10 \mathrm{Gyr}\left(R_{\mathrm{apo}}=152_{-43}^{+65} \mathrm{kpc}\right.$ and $\left|Z_{\max }\right|=$ $\left.75_{-13}^{+26} \mathrm{kpc}\right)$. CS $22941-012$, in contrast, has orbital parameters $R_{\text {apo }}=26_{-6}^{+3} \mathrm{kpc}$ and $\left|Z_{\max }\right|=4.3 \pm 0.6 \mathrm{kpc}$. While the evolutionary origin of BMP binary stars is not in doubt, it is interesting that CS 22966-043, a star with such a bizarre chemical composition, has an extreme orbit that is consistent with the accretion scenario. Meanwhile the RV-constant BMP star, CS 22941-012, appears to be on a more normal outer halo orbit.

\subsubsection{Dwarf Spheroidal Systems}

The number of dSph systems with known Galactic orbital parameters is growing, thanks to careful measurements of their proper motions. The majority of the present-day dSphs with known proper motions and orbital parameters do not approach closer than a few tens of kpc from the Galactic center or the Solar neighborhood (Canis Major: $R_{\text {peri }}=10.5 \pm 0.9 \mathrm{kpc}$, Dinescu et al. 2005; Carina: $R_{\text {peri }}=20_{-17}^{+43} \mathrm{kpc}$, Piatek et al. 2003; Fornax: $\quad R_{\text {peri }}=138 \pm 19 \mathrm{kpc}$, Dinescu et al. 2004; $R_{\text {peri }}=118_{-52}^{+19} \mathrm{kpc}$, Piatek et al. 2007; Sculptor: $R_{\text {peri }}=$ $120 \pm 51 \mathrm{kpc}$, Dinescu et al. 2004; $R_{\text {peri }}=68_{-37}^{+15} \mathrm{kpc}$, Piatek et al. 2006; Ursa Minor: $R_{\text {peri }}=40_{-30}^{+36} \mathrm{kpc}$, Piatek et al. 2005). Thus it is unlikely that these specific systems could have contributed any significant fraction of the local stellar halo. Additionally, the Leo II dSph system has not undergone significant tidal interaction with the Milky Way Galaxy, is currently at a very great distance $(218 \mathrm{kpc})$, and therefore might be on a 
more circular Galactic orbit that does not approach near to the Galactic center (Siegel et al. 2008). The orbital periods of the dSph systems are typically several Gyr, implying that they will have reached their perigalactica no more than a few times, and even the systems that do venture near the Solar radius would only spend a small fraction of their orbital periods there.

Not coincidentally, this conclusion was also reached from comparison of the detailed abundances of several of these same dSphs (Carina, Fornax, Leo II, Sculptor, and Ursa Minor) with halo stars of the same metallicity ranges (Venn et al. 2004; Shetrone et al. 2008). One exception is the Sagittarius dSph, whose leading tidal arm has deposited debris within a few kpc of the present Solar neighborhood (Majewski et al. 2003). The stars that Sagittarius is presently contributing to the halo are different than the stars in its residual core (Bellazzini et al. 2006; Chou et al. 2007; Siegel et al. 2007), signaling that $\mathrm{dSph}$ systems of the present day may not resemble the stars that they have already lost to the stellar halo of the Galaxy.

While the growing consensus points to few accreted dSph stars constituting the local metal-poor stellar halo, chemical and kinematic analysis of large samples of in situ halo stars will be needed to determine if a significant fraction of the true outer halo (e.g., stars that never approach nearer to the Galactic center than, say, $20 \mathrm{kpc}$ ) is comprised of the remnants of former or present dSph systems. This is a daunting observational challenge, but the results from such a study would be extremely interesting.

\section{KINEMATICS AS A FUNCTION OF ABUNDANCES}

\section{1. $\alpha$-poor Stars}

Most metal-poor stars exhibit $[\alpha / \mathrm{Fe}]$ ratios that are 0.3-0.4 dex above the Solar ratio (e.g., Wallerstein 1962; Edvardsson et al. 1993; McWilliam et al. 1995b). A handful of metal-poor stars, however, have $[\alpha / \mathrm{Fe}]$ ratios that are significantly lower than the standard plateau, and a few such stars have $[\alpha / \mathrm{Fe}]$ ratios that are well below the Solar ratio (e.g., Carnev et al. 1997; Ivans et al. 2003). Similar $\alpha$ deficiencies have been observed in several nearby dSph galaxies Bonifacio et al. 2000a; Shetrone et al. 2001, 2003; Tolstoy et al. 2003; Fulbright et al. 2004; Sadakane et al. 2004; Geisler et al. 2005; Sbordone et al. 2007; Koch et al. 2008; Shetrone et al. 2008), prompting speculation that the $\alpha$-poor stars may be signatures of past accretion of dSphs. Furthermore, stars associated with the Sagittarius dSph, which is presently interacting with the Milky Way (Ibata et al. 1994; Majewski et al. 2003), are very dissimilar to field stars and are decidedly underabundant in $\alpha$ - and Fe-peak elements (at least in the metallicity range covered, $-1.0<[\mathrm{Fe} / \mathrm{H}]<+0.0$; Bonifacio et al. 2000a; McWilliam \& Smecker-Hane 2005; Sbordone et al. 2007). Only a few $\alpha$-poor field stars are known, and for this small subset of the halo the accretion hypothesis deserves additional scrutiny. With the recent report of a handful of new $\alpha$-poor metal-poor stars (Barklem et al. 2005) from the stellar content of the Hamburg-ESO Survey (Frebel et al. 2006; Christlieb et al. 2008), we are in a position to reevaluate the kinematic properties of these stars to search for clues of their origin.
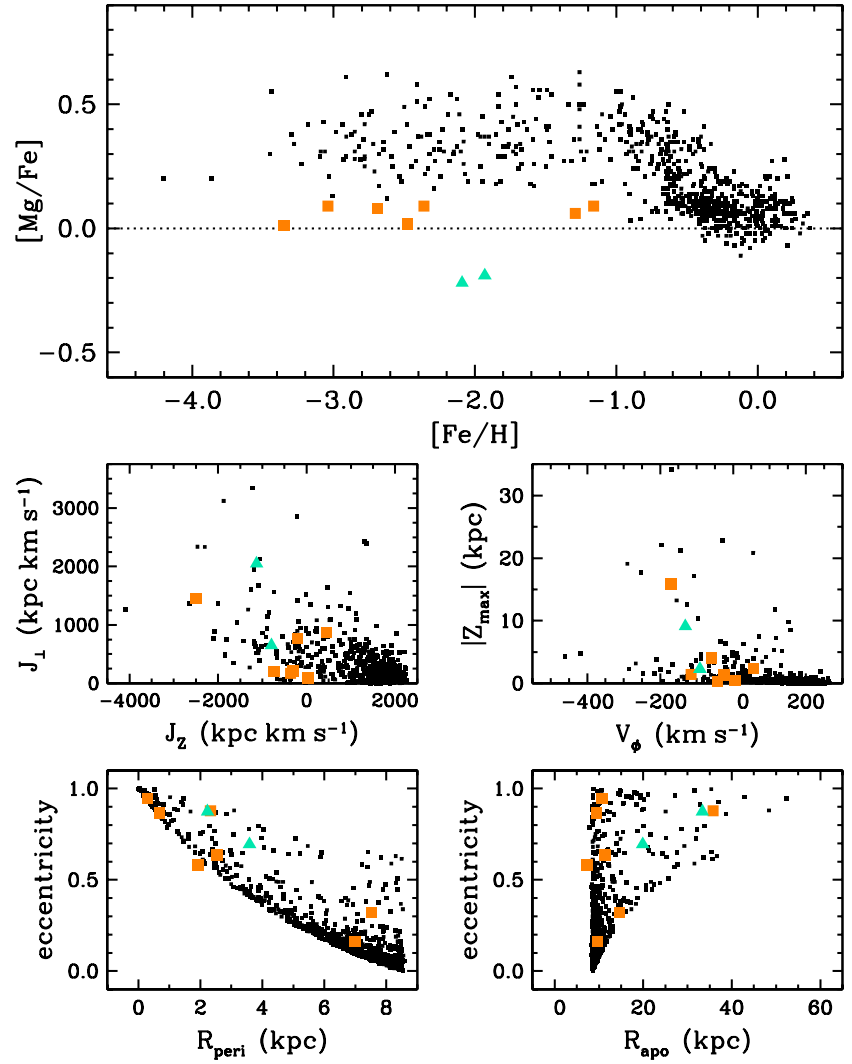

FIG. 21. - Defining stars of low $[\mathrm{Mg} / \mathrm{Fe}]$. Restricting our $\mathrm{Mg}$ poor sample to stars with $[\mathrm{Fe} / \mathrm{H}]<-1.0$, filled squares (orange in the online edition) represent stars with $+0.0<[\mathrm{Mg} / \mathrm{Fe}]<+0.1$ and filled triangles (turquoise in the online edition) represent stars with $[\mathrm{Mg} / \mathrm{Fe}]<+0.0$. The stars selected according to these definitions are highlighted in the lower four panels. [Please see the electronic edition for a color version of this figure.]

In the top panel of Figure 21 we identify two classes of $\alpha$-poor stars: those with $+0.0 \leq[\mathrm{Mg} / \mathrm{Fe}]<+0.1$ (" $\alpha$ deficient") and those with $[\mathrm{Mg} / \mathrm{Fe}]<+0.0$ (" $\alpha$-poor"). For purposes of this analysis we also restrict our sample to stars with $[\mathrm{Fe} / \mathrm{H}]<-1.0$. In the bottom panels of Figure 21 we display the kinematic and orbital properties of these stars. They share no common region in phase space. Several orbits extend to large distances above the Galactic plane or to large radii, but no kinematic signature is preferred. This suggests that at least a very small fraction of Galactic halo stars formed in chemically inhomogeneous regions that were deficient (and, allegedly, deficient by varying degrees) in the $\alpha$ elements. Ivans et al. (2003) performed an exhaustive comparison of the abundance patterns of three $\alpha$-poor stars to Type Ia SN models. They concluded that these stars seem to have increased contributions from Type Ia yields (relative to other stars at their metallicity), yet they cautioned that no model could reproduce the overall abundance patterns of these stars. They speculated that these stars may have been among the earliest to from from Type Ia products, though they could also not rule out the accretion hypothesis.

A number of studies (Unavane et al. 1996, Fulbright 2002, Venn et al. 2004, Pritzl et al. 2005, and Geisler et al. 2007, in addition to those listed above) have concluded that the chemical compositions of $\mathrm{dSph}$ stars and halo giants are sufficiently different, and only a small fraction of the Milky Way stellar halo can 
be composed of stars accreted from dSphs chemically similar to those surviving to the present day. Our result does not imply that the $\alpha$-poor stars could not have formed in dSph systems that were assumed into the Milky Way. Since many of these stars are on eccentric orbits that take them to large Galactic radii, we may surmise that either these inhomogeneous regions were located at large Galactic radii (perhaps before the bulk of the Galaxy formed) or the stars were formed in (separate?) dSphs and accreted at some time in the past.

\subsection{Stars with Specific n-capture Enrichment Signatures}

Can the $n$-capture material present in these metal-poor stars be used to identify any preferred kinematics of the parent clouds from which they formed? Given the different mass ranges of the stars commonly thought to produce $n$-capture material, this is an attractive possibility if the IMF's of the parent clouds differ significantly.

In the top panel of Figure 22, we identify stars that have either a nearly pure $s$-process or $r$-process $[\mathrm{Ba} / \mathrm{Eu}]$ ratio. Pure $r$-process enrichment (or, viewed another way, lack of appreciable $s$-process enrichment) occurs over a very wide range of metallicity $(-3.0<[\mathrm{Fe} / \mathrm{H}]-0.4$, or a factor of $\approx 400$ in $\mathrm{Fe} / \mathrm{H}-\mathrm{a}$ fact which is remarkable in its own right!). Our sample includes a rather small number of stars exhibiting a pure $s$-process signature; this is a consequence of our (somewhat arbitrary) choice of literature data to include. In the lower panels of Figure 22, we show the kinematic properties of these stars. The stars dominated by $r$-process $n$-capture enrichment show no preferred kinematic properties - they scatter over all values of $V_{\phi},\left|Z_{\max }\right|, R_{\text {peri }}$, and $R_{\text {apo }}$. The three stars exhibiting a pure $s$-process signature do not indicate any kinematic preferences, either.

The main $r$-process produces a robust abundance pattern for species with $Z \geq 56$ (e.g., Sneden et al. 2003a; Ivans et al. 2006; Cowan \& Sneden 2006; Roederer et al. 2008), but it has become clear over the last decade that this unique signature does not apply to the lighter $n$-capture nuclei, such as the Sr-Y-Zr group. Close analyses of two metal-poor stars, HD 88609 (Honda et al. 2007) and HD 122563 (Honda et al. 2006), reveal an abundance pattern that gradually declines with increasing $Z$, producing high ratios between light and heavy $n$-capture species (see also McWilliam et al. 1995b, Wasserburg et al. 1996, Johnson \& Bolte 2002, Aoki et al. 2005, Lai et al. 2007, and Lai et al. 2008). This pattern cannot be associated with the main $r$-process, the main $s$-process, or the weak $s$-process, and could be representative of - for example - a light element primary process (Travaglio et al. 2004), a rapid proton-capture process (Wanajo 2006), a weak $r$-process (Wanajo \& Ishimaru 2006), a cold $r$-process (Wanajo 2007), hypernovae (Qian \& Wasserburg 2008), or a high entropy wind from Type II SNe (Farouqi et al. 2008). This multiplicity of scenarios, with a variety of proposed SN mass ranges responsible for the creation of the light $n$-capture elements, demands additional observational constraints. Once again, if the IMF of a certain proto-stellar metal-poor cloud was significantly different from other clouds, this could manifest itself in the de-
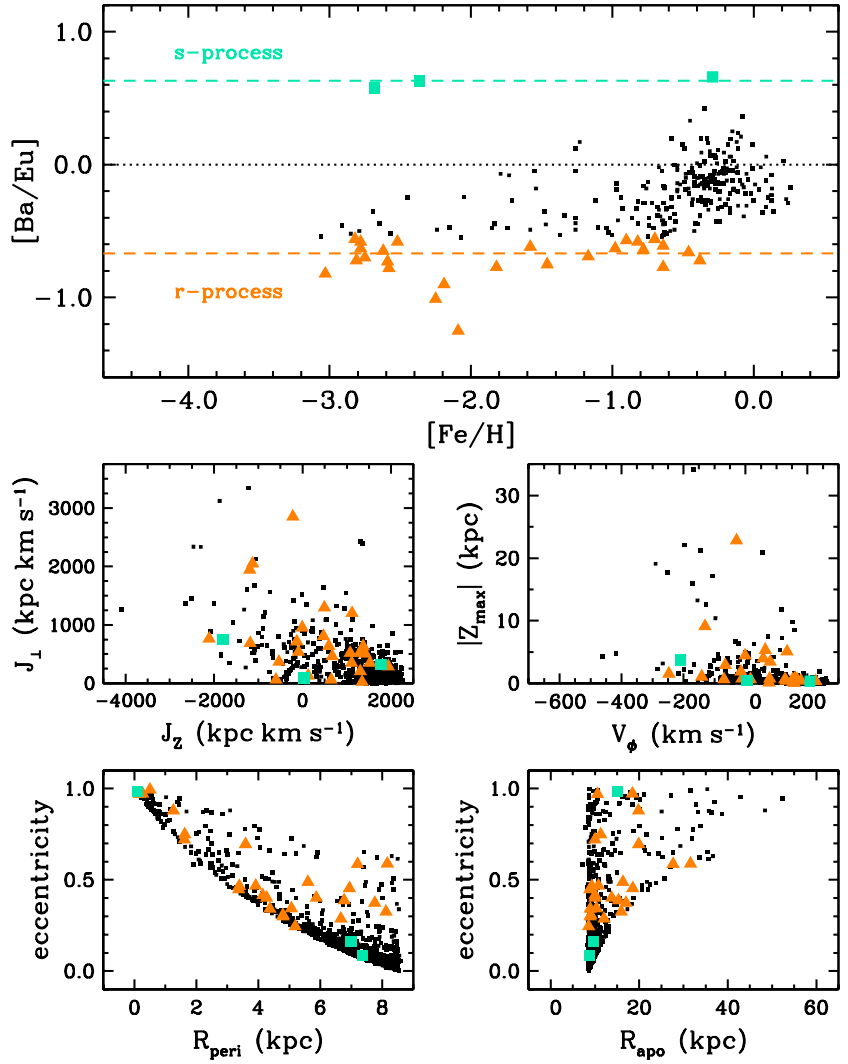

FIG. 22.- Defining stars exhibiting pure $r$-process and pure $s$-process $[\mathrm{Ba} / \mathrm{Eu}]$ ratios. The lower dashed line (orange in the online edition) represents the pure $r$-process ratio $(-0.67)$ and the upper dashed line (turquoise in the online edition) represents the pure $s$-process ratio $(+0.63)$ as predicted by Simmerer et al. (2004) . Filled triangles (orange in the online edition) represent stars with $[\mathrm{Ba} / \mathrm{Eu}]<-0.55$ and filled squares (turquoise in the online edition) represent stars with $[\mathrm{Ba} / \mathrm{Eu}]>+0.50$. The stars selected according to these definitions are highlighted in the lower four panels. [Please see the electronic edition for a color version of this figure.]

rived $[\mathrm{Ba} / \mathrm{Y}]$ ratios. In the top panel of Figure 23 we identify stars with low $[\mathrm{Ba} / \mathrm{Y}]$ ratios $([\mathrm{Ba} / \mathrm{Y}]<-0.55)$ and low $[\mathrm{Ba} / \mathrm{Fe}]$ ratios $([\mathrm{Ba} / \mathrm{Fe}]<-0.50)$, similar to the abundances found in HD 88609 and HD $122563 .{ }^{8}$ Again, though, these stars exhibit no clear kinematic properties, although they may slightly prefer eccentric orbits, which could point to a common - though yet poorly constrained - origin. (The common retrograde orbits are a result of the narrow $[\mathrm{Fe} / \mathrm{H}]$ range of this sub-sample, and are not of physical consequence.)

At low metallicities, $n$-capture enrichment is probably a very localized phenomenon that results in a wide distribution of $n$-capture abundances, and thus it will be extremely difficult to identify any associated largescale kinematic behaviors of the proto-stellar clouds from which these stars formed. The potential gain from such a connection, however, justifies the continued effort toward this goal.

\subsection{The Most Metal-Poor Stars Known}

We also examine the kinematic properties of the three most metal-poor stars known, HE 0107-5240 $([\mathrm{Fe} / \mathrm{H}]=$

8 We note, as Honda et al. (2007) have, that high [Sr/Ba] or $[\mathrm{Y} / \mathrm{Ba}]$ ratios do not uniquely define the abundance pattern associated with this process, since HD 88609 was selected for analysis based on its $[\mathrm{Sr} / \mathrm{Ba}]$ ratio, identical to that of HD 122563. 


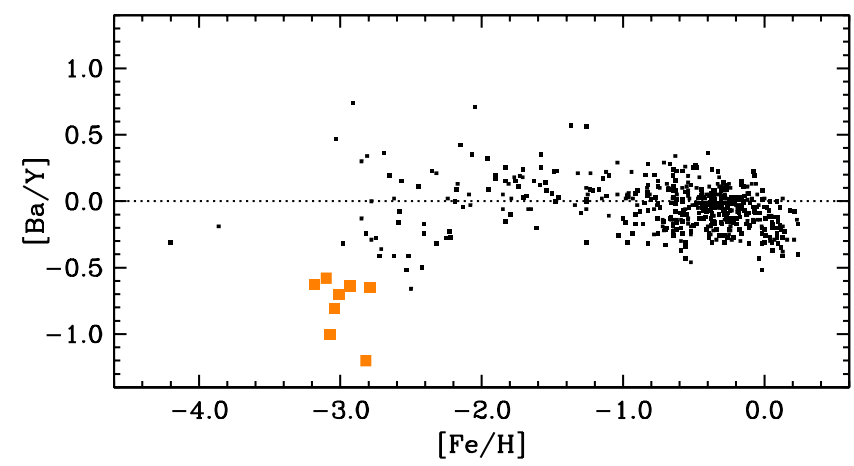

point to a very extreme orbit for HE 1327-2326, which is perhaps not unexpected given the uncharacteristically low metal abundance of this interesting star. Precise proper motion measurements for HE $0107-5240$ and HE 0577-4840 will be necessary to determine whether extreme orbits are a common characteristic of the most metal-poor stars in the Galaxy. Furthermore, HE 1327-2326 spends a very small fraction of its time in the inner regions of the Galaxy, suggesting, perhaps, that many more objects with compositions similar to this star currently reside in the distant realms of the stellar halo. This again hints to the possibility that objects in the true outer halo of the Galaxy may bear little resemblance to those that inhabit the inner few tens of kpc.

\section{CONCLUSIONS}

We have compiled a large sample of metal-poor stars with reported abundances from the literature. For the subset of these stars with reliable proper motion measurements, we have computed space velocities and Galactic orbital parameters. We have used the combination of chemical and kinematic information to identify any abundance patterns that are common to stars with similar kinematics or identify any kinematic signatures that are common to stars with similar chemical enrichment patterns. Our major results can be summarized as follows.

(1) A proper motion selection criterion for our sample biases the very metal-poor end of our sample toward stars with large proper motions and hence extreme orbits, many of which are on retrograde Galactic orbits. This bias must be borne in mind when interpreting chemical enrichment patterns in very metal-poor stars.

(2) We find no abundance trends with maximum radial distance from the Galactic center or maximum vertical distance above the Galactic disk. Current highresolution abundance analyses are limited to sampling halo stars that happen to be passing relatively near to the Solar neighborhood. Other studies have found very slight decreases in abundance ratios with increasing distance from the Galactic center, and this issue may remain unsettled until large, deep abundance surveys and proper motion surveys can reach beyond the Solar neighborhood into a true in situ sample of the stellar halo.

(3) We use only kinematic criteria to define our inner halo (stars on prograde, eccentric orbits that do not stray beyond $15 \mathrm{kpc}$ from the Galactic center or $5 \mathrm{kpc}$ from the Galactic plane) and outer halo (stars on very retrograde orbits or stars whose orbits reach more than $25 \mathrm{kpc}$ from the Galactic center or more than $10 \mathrm{kpc}$ above the Galactic plane) samples, based on the kinematic properties of these populations derived from much larger datasets. In the metallicity regimes where the two populations overlap, roughly $-2.5<[\mathrm{Fe} / \mathrm{H}]<-1.5$, the $[\mathrm{Mg} / \mathrm{Fe}]$ ratio of the outer halo may be lower than the inner halo by $\sim 0.1$ dex. For $[\mathrm{Ni} / \mathrm{Fe}]$ and $[\mathrm{Ba} / \mathrm{Fe}]$ the star-to-star abundance scatter of the inner halo is consistently smaller than the star-to-star abundance scatter of the outer halo. The $[\mathrm{Na} / \mathrm{Fe}],[\mathrm{Ca} / \mathrm{Fe}],[\mathrm{Ti} / \mathrm{Fe}]$, and $[\mathrm{Y} / \mathrm{Fe}]$ ratios of both populations show similar levels of scatter. We do not have enough $[\mathrm{Eu} / \mathrm{Fe}]$ measurements in our sample to draw any conclusions from this abundance ratio.

(4) Our inner halo population appears chemically ho- 
mogeneous, suggesting that a significant fraction of the Milky Way stellar halo had a common origin from a wellmixed ISM.

(5) In contrast, our kinematically diverse outer halo population is also chemically diverse, suggesting that another significant fraction of the Milky Way stellar halo formed in remote regions where chemical enrichment was dominated by local SN events. This component is reminiscent of the "chaotic origin" for the Galaxy suggested by the globular cluster data of Searle \& Zinn (1978).

(6) If we classify globular clusters by these same kinematic criteria, all of the inner halo and most of the outer halo clusters follow similar (mean) abundance trends with comparable degrees of scatter to the inner halo population of field stars. The chemical similarity of the inner halo and the globular clusters may suggest that these abundance trends represent the time-averaged mean abundances of a metal-poor ISM pre-enriched by the earliest generations of stars. We find no mean cluster abundance dependence with increasing radial distance from the Galactic center or vertically from the Galactic plane.

(7) We find no kinematic signature in common to groups of metal-poor stars with peculiar abundance patters $(\alpha$-poor stars, stars showing a pure- $s$ - or pure- $r$ process $n$-capture enrichment pattern, or stars with a deficiency of heavy $n$-capture material).

(8) Our results do not exclude the possibility that any of these individual stars were accreted by the Milky Way from, e.g., dSph systems; however, the orbits of many present-day dSphs (especially those whose stars have been subject to high-resolution abundance analyses) rarely bring them near the Solar radius, so they would not be expected to contribute many stars to the local metal-poor stellar halo.

(9) Several individual stars - including the most metalpoor star known - and dSph systems whose compositions differ greatly from the bulk of the stellar halo in the Solar neighborhood have long orbital periods ( few Gyr) and extreme orbital characteristics $\left(R_{\text {apo }} \sim\right.$ few hundred $\mathrm{kpc}$ ). If stars like these spend the majority of their time in the distant regions of the Milky Way stellar halo, this raises the possibility that many more stars with unusual abundance patterns may occupy the true outer halo of the Galaxy, which may have little resemblance to the local stellar halo.

The abundance dataset used for this analysis was compiled from a variety of literature sources, and the inherent systematic differences from one study to another limit our ability to detect more subtle chemical differences than those described above. Large, metal-poor stellar samples from which abundances are derived in a homogeneous manner are necessary to perform a more detailed nucleo-kinematic analysis. The construction and analysis of such datasets is presently underway.

Evidence continues to grow in support of the notion that the chemical enrichment history of the Milky Way stellar halo is nonuniform on both small and large scales. While it is unlikely that an exact correlation between the kinematic properties of a star and its chemical abundance pattern will ever be identified, we may be approaching an era where it is necessary to know the kinematic properties of a field halo star in order to place its chemical abundance pattern in the proper nucleosynthetic context. The challenge for future studies will be to articulate the degree of chemical dissimilarity in the Milky Way halo kinematic substructure.

It is a pleasure to thank Anna Frebel and Chris Sneden for many extensive discussions relating to this topic and detailed comments on assorted versions of this manuscript. I. U. R. also thanks John Cowan, George Preston, and Matthew Shetrone for their helpful suggestions and encouragement, as well as David Lambert, who originally suggested this literature study. Additional thanks go to Monique Spite for sending radial velocity measurements for the "First Stars" sample in advance of publication, Douglas Lin for sharing a copy his orbit integrator code, and the anonymous referee for helpful suggestions. This research would not have been possible without the resources available from the SIMBAD and VizieR (Ochsenbein et al. 2000) online databases, operated at CDS, Strasbourg, France, the Two Micron AllSky Survey, and NASA's Astrophysics Data System Bibliographic Services. Funding has been provided by NSF grant AST 06-07708 (to C. Sneden).

\section{REFERENCES}

Allende Prieto, C., et al. 2007, ArXiv e-prints, 710, arXiv:0710.5780 Aoki, W., et al. 2005, ApJ, 632, 611

Aoki, W., et al. 2006, ApJ, 639, 897

Argast, D., Samland, M., Gerhard, O. E., \& Thielemann, F.-K. 2000, A\&A, 356, 873

Arnone, E., Ryan, S. G., Argast, D., Norris, J. E., \& Beers, T. C. 2005, A\&A, 430, 507

Audouze, J., \& Tinsley, B. M. 1976, ARA\&A, 14, 43

Barklem, P. S., et al. 2005, A\&A, 439, 129

Beers, T. C., \& Sommer-Larsen, J. 1995, ApJS, 96, 175

Beers, T. C., Chiba, M., Yoshii, Y., Platais, I., Hanson, R. B., Fuchs, B., \& Rossi, S. 2000, AJ, 119, 2866

Beers, T. C., \& Christlieb, N. 2005, ARA\&A, 43, 531

Bell, E. F., et al. 2008, ApJ, 680, 295

Bellazzini, M., Newberg, H. J., Correnti, M., Ferraro, F. R., \& Monaco, L. 2006, A\&A, 457, L21

Bensby, T., Feltzing, S., \& Lundström, I. 2003, A\&A, 410, 527

Bonifacio, P., Hill, V., Molaro, P., Pasquini, L., Di Marcantonio, P., \& Santin, P. 2000a, A\&A, 359, 663

Bonifacio, P., Monai, S., \& Beers, T. C. 2000b, AJ, 120, 2065

Bonifacio, P., \& Caffau, E. 2003, A\&A, 399, 1183
Burbidge, E. M., Burbidge, G. R., Fowler, W. A., \& Hoyle, F. 1957, Reviews of Modern Physics, 29, 547

Busso, M., Gallino, R., \& Wasserburg, G. J. 1999, ARA\&A, 37, 239

Carney, B. W., Laird, J. B., Latham, D. W., \& Aguilar, L. A. 1996, AJ, 112,668

Carney, B. W., Wright, J. S., Sneden, C., Laird, J. B., Aguilar, L. A., \& Latham, D. W. 1997, AJ, 114, 363

Carney, B. W., Latham, D. W., \& Laird, J. B. 2005, AJ, 129, 466

Carollo, D., et al. 2007, Nature, 450, 1020

Carretta, E., Gratton, R., Cohen, J. G., Beers, T. C., \& Christlieb, N. 2002, AJ, 124, 481

Cayrel, R., et al. 2004, A\&A, 416, 1117

Chiba, M., \& Yoshii, Y. 1998, AJ, 115, 168

Chiba, M., \& Beers, T. C. 2000, AJ, 119, 2843

Chou, M.-Y., et al. 2007, ApJ, 670, 346

Christlieb, N., et al. 2002, Nature, 419, 904

Christlieb, N., Gustafsson, B., Korn, A. J., Barklem, P. S., Beers, T. C., Bessell, M. S., Karlsson, T., \& Mizuno-Wiedner, M. 2004, ApJ, 603, 708

Christlieb, N., Schörck, T., Frebel, A., Beers, T. C., Wisotzki, L., \& Reimers, D. 2008, A\&A, 484, 721 
Cohen, J. G. 2004, AJ, 127, 1545

Cohen, J. G., et al. 2004, ApJ, 612, 1107

Cowan, J. J., \& Sneden, C. 2006, Nature, 440, 1151

Cutri, R. M., et al. 2003, The IRSA 2MASS All-Sky Point Source Catalog, NASA/IPAC Infrared Science Archive

Demarque, P., Woo, J.-H., Kim, Y.-C., \& Yi, S. K. 2004, ApJS, 155,667

Dinescu, D. I., Girard, T. M., \& van Altena, W. F. 1999, AJ, 117, 1792

Dinescu, D. I., Majewski, S. R., Girard, T. M., \& Cudworth, K. M. 2000, AJ, 120, 1892

Dinescu, D. I., Majewski, S. R., Girard, T. M., \& Cudworth, K. M. 2001, AJ, 122, 1916

Dinescu, D. I. 2002, Omega Centauri, A Unique Window into Astrophysics, 265, 365

Dinescu, D. I., Girard, T. M., van Altena, W. F., \& López, C. E. 2003, AJ, 125, 1373

Dinescu, D. I., Keeney, B. A., Majewski, S. R., \& Girard, T. M. 2004, AJ, 128, 687

Dinescu, D. I., Martínez-Delgado, D., Girard, T. M., Peñarrubia, J., Rix, H.-W., Butler, D., \& van Altena, W. F. 2005, ApJ, 631 L49

Edvardsson, B., Andersen, J., Gustafsson, B., Lambert, D. L., Nissen, P. E., \& Tomkin, J. 1993, A\&A, 275, 101

Eggen, O. J., Lynden-Bell, D., \& Sandage, A. R. 1962, ApJ, 136, 748

Farouqi, K., et al. 2008, ApJ, submitted

Font, A. S., Johnston, K. V., Bullock, J. S., \& Robertson, B. E. 2006, ApJ, 638, 585

Fowler, W. A., Caughlan, G. R., \& Zimmerman, B. A. 1967, ARA\&A, 5, 525

Francois, P., et al. 2007, A\&A, 476, 935

Frebel, A., et al. 2005, Nature, 434, 871

Frebel, A., et al. 2006, ApJ, 652, 1585

Frebel, A., Collet, R., Eriksson, K., Christlieb, N., \& Aoki, W. 2008a, ApJ, 684, 588

Frebel, A., Johnson, J. L., \& Bromm, V. 2008b, MNRAS, submitted

Fulbright, J. P. 2000, AJ, 120, 1841

Fulbright, J. P. 2002, AJ, 123, 404

Fulbright, J. P. 2004, Origin and Evolution of the Elements, 4, 22

Fulbright, J. P., Rich, R. M., \& Castro, S. 2004, ApJ, 612, 447

Geisler, D., Smith, V. V., Wallerstein, G., Gonzalez, G., \& Charbonnel, C. 2005, AJ, 129, 1428

Geisler, D., Wallerstein, G., Smith, V. V., \& Casetti-Dinescu, D. I. 2007, PASP, 119, 939

Gilmore, G., Wyse, R. F. G., \& Kuijken, K. 1989, ARA\&A, 27, 555

Gilmore, G., Wyse, R. F. G., \& Norris, J. E. 2002, ApJ, 574, L39

Gratton, R. G., Carretta, E., Desidera, S., Lucatello, S., Mazzei, P., \& Barbieri, M. 2003, A\&A, 406, 131

Gratton, R., Sneden, C., \& Carretta, E. 2004, ARA\&A, 42, 385

Hanson, R. B., Sneden, C., Kraft, R. P., \& Fulbright, J. 1998, AJ, 116,1286

Harris, W. E. 1996, AJ, 112, 1487

Hartwick, F. D. A. 1976, ApJ, 209, 418

Helmi, A., \& White, S. D. M. 1999, MNRAS, 307, 495

Helmi, A. 2008, A\&A Rev., 15, 145

Hill, V., et al. 2002, A\&A, 387, 560

Honda, S., et al. 2004a, ApJS, 152, 113

Honda, S., Aoki, W., Kajino, T., Ando, H., Beers, T. C., Izumiura, H., Sadakane, K., \& Takada-Hidai, M. 2004b, ApJ, 607, 474

Honda, S., Aoki, W., Ishimaru, Y., Wanajo, S., \& Ryan, S. G. 2006 ApJ, 643, 1180

Honda, S., Aoki, W., Ishimaru, Y., \& Wanajo, S. 2007, ApJ, 666, 1189

Ibata, R. A., Gilmore, G., \& Irwin, M. J. 1994, Nature, 370, 194

Iben, I., Jr. 1983, Memorie della Societa Astronomica Italiana, 54, 321

Ivans, I. I., Kraft, R. P., Sneden, C., Smith, G. H., Rich, R. M., \& Shetrone, M. 2001, AJ, 122, 1438

Ivans, I. I., Sneden, C., James, C. R., Preston, G. W., Fulbright, J. P., Höflich, P. A., Carney, B. W., \& Wheeler, J. C. 2003, ApJ, 592, 906

Ivans, I. I., Simmerer, J., Sneden, C., Lawler, J. E., Cowan, J. J., Gallino, R., \& Bisterzo, S. 2006, ApJ, 645, 613

Ivezić, Ž., et al. 2008, ApJ, 684, 287

Johnson, J. A., \& Bolte, M. 2002, ApJ, 579, 616
Johnston, K. V., Hernquist, L., \& Bolte, M. 1996, ApJ, 465, 278 Johnston, K. V. 1998, ApJ, 495, 297

Kepley, A. A., et al. 2007, AJ, 134, 1579

Kerr, F. J., \& Lynden-Bell, D. 1986, MNRAS, 221, 1023

Kinman, T. D., Cacciari, C., Bragaglia, A., Buzzoni, A., \& Spagna, A. 2007, MNRAS, 375, 1381

Koch, A., Grebel, E. K., Gilmore, G. F., Wyse, R. F. G., Kleyna, J. T., Harbeck, D. R., Wilkinson, M. I., \& Wyn Evans, N. 2008, AJ, 135,1580

Korn, A. J., Mashonkina, L., Richard, O., Frebel, A., Aoki, W., \& Christlieb, N. 2008, First Stars III, 990, 167

Kraft, R. P. 1979, ARA\&A, 17, 309

Lai, D. K., Johnson, J. A., Bolte, M., \& Lucatello, S. 2007, ApJ, 667,1185

Lai, D. K., Bolte, M., Johnson, J. A., Lucatello, S., Heger, A., \& Woosley, S. E. 2008, ApJ, 681, 1524

Lee, J.-W., \& Carney, B. W. 2002, AJ, 124, 1511

Lee, J.-W., Carney, B. W., \& Habgood, M. J. 2005, AJ, 129, 251

Lee, Y. S., et al. 2007, ArXiv e-prints, 710, arXiv:0710.5645

Majewski, S. R. 1992, ApJS, 78, 87

Majewski, S. R., Munn, J. A., \& Hawley, S. L. 1996, ApJ, 459, L73

Majewski, S. R., Skrutskie, M. F., Weinberg, M. D., \& Ostheimer, J. C. 2003, ApJ, 599, 1082

Martin, N. F., Ibata, R. A., Bellazzini, M., Irwin, M. J., Lewis, G. F., \& Dehnen, W. 2004, MNRAS, 348, 12

McWilliam, A., Preston, G. W., Sneden, C., \& Shectman, S. 1995a, AJ, 109, 2736

McWilliam, A., Preston, G. W., Sneden, C., \& Searle, L. 1995b, AJ, 109, 2757

McWilliam, A. 1997, ARA\&A, 35, 503

McWilliam, A. 1998, AJ, 115, 1640

McWilliam, A., \& Smecker-Hane, T. A. 2005, ApJ, 622, L29

McCall, M. L. 2004, AJ, 128, 2144

McCarthy, J. K., \& Nemec, J. M. 1997, ApJ, 482, 203

Miceli, A., et al. 2008, ApJ, 678, 865

Mihalas, D., \& Binney, J. 1981, San Francisco, CA, W. H. Freeman and Co., 608

Monet, D. G., et al. 2003, AJ, 125, 984

Morrison, H. L., et al. 2008, ArXiv e-prints, 804, arXiv:0804.2448

Mottini, M., Wallerstein, G., \& McWilliam, A. 2008, AJ, 136, 614

Newberg, H. J., \& Sloan Digital Sky Survey Collaboration 2003,

BAAS, 35, 1385

Nissen, P. E., \& Schuster, W. J. 1997, A\&A, 326, 751

Norris, J. 1986, ApJS, 61, 667

Norris, J. E., \& Ryan, S. G. 1989, ApJ, 340, 739

Norris, J. E. 1994, ApJ, 431, 645

Norris, J. E., \& Da Costa, G. S. 1995, ApJ, 447, 680

Norris, J. E., Freeman, K. C., \& Mighell, K. J. 1996, ApJ, 462, 241

Norris, J. E., Freeman, K. C., Mayor, M., \& Seitzer, P. 1997, ApJ, 487, L187

Norris, J. E., Christlieb, N., Korn, A. J., Eriksson, K., Bessell, M. S., Beers, T. C., Wisotzki, L., \& Reimers, D. 2007, ApJ, 670, 774

Ochsenbein, F., Bauer, P., \& Marcout, J. 2000, A\&AS, 143, 23

Perryman, M. A. C., \& ESA 1997, ESA Special Publication, 1200

Piatek, S., Pryor, C., Olszewski, E. W., Harris, H. C., Mateo, M., Minniti, D., \& Tinney, C. G. 2003, AJ, 126, 2346

Piatek, S., Pryor, C., Bristow, P., Olszewski, E. W., Harris, H. C., Mateo, M., Minniti, D., \& Tinney, C. G. 2005, AJ, 130, 95

Piatek, S., Pryor, C., Bristow, P., Olszewski, E. W., Harris, H. C., Mateo, M., Minniti, D., \& Tinney, C. G. 2006, AJ, 131, 1445

Piatek, S., Pryor, C., Bristow, P., Olszewski, E. W., Harris, H. C., Mateo, M., Minniti, D., \& Tinney, C. G. 2007, AJ, 133, 818

Preston, G. W., Shectman, S. A., \& Beers, T. C. 1991, ApJ, 375, 121

Preston, G. W., Beers, T. C., \& Shectman, S. A. 1994, AJ, 108, 538

Preston, G. W., \& Sneden, C. 2000, AJ, 120, 1014

Pritzl, B. J., Venn, K. A., \& Irwin, M. 2005, AJ, 130, 2140

Prochaska, J. X., Naumov, S. O., Carney, B. W., McWilliam, A., \& Wolfe, A. M. 2000, AJ, 120, 2513

Qian, Y.-Z., \& Wasserburg, G. J. ApJ preprint doi: $10.1086 /$ ' 591545 '

Reddy, B. E., Tomkin, J., Lambert, D. L., \& Allende Prieto, C. 2003, MNRAS, 340, 304

Roederer, I. U., Lawler, J. E., Sneden, C., Cowan, J. J., Sobeck, J. S., \& Pilachowski, C. A. 2008, ApJ, 675, 723

Ryan, S. G., \& Norris, J. E. 1991a, AJ, 101, 1835 
Ryan, S. G., \& Norris, J. E. 1991b, AJ, 101, 1865

Sadakane, K., Arimoto, N., Ikuta, C., Aoki, W., Jablonka, P., \& Tajitsu, A. 2004, PASJ, 56, 1041

Sandage, A. 1986, ARA\&A, 24, 421

Sandage, A., \& Fouts, G. 1987, AJ, 93, 74

Sbordone, L., Bonifacio, P., Buonanno, R., Marconi, G., Monaco, L., \& Zaggia, S. 2007, A\&A, 465, 815

Schlegel, D. J., Finkbeiner, D. P., \& Davis, M. 1998, ApJ, 500, 525

Schörck, T., et al. 2008, A\&A, submitted

Searle, L., \& Zinn, R. 1978, ApJ, 225, 357

Seeger, P. A., Fowler, W. A., \& Clayton, D. D. 1965, ApJS, 11, 121

Shetrone, M. D., Côté, P., \& Sargent, W. L. W. 2001, ApJ, 548, 592

Shetrone, M., Venn, K. A., Tolstoy, E., Primas, F., Hill, V., \& Kaufer, A. 2003, AJ, 125, 684

Shetrone, M. D., Siegel, M. H., Cook, D. O., \& Bosler, T. 2008, AJ, in press

Siegel, M. H., et al. 2007, ApJ, 667, L57

Siegel, M. H., Majewski, S. R., Sohn, S. T., Shetrone, M. D., Munoz, R. R., \& Patterson, R. J. 2008, ApJ, submitted

Simmerer, J., Sneden, C., Cowan, J. J., Collier, J., Woolf, V. M., \& Lawler, J. E. 2004, ApJ, 617, 1091

Sneden, C., Kraft, R. P., Shetrone, M. D., Smith, G. H., Langer, G. E., \& Prosser, C. F. 1997, AJ, 114, 1964

Sneden, C., Johnson, J., Kraft, R. P., Smith, G. H., Cowan, J. J., \& Bolte, M. S. 2000, ApJ, 536, L85

Sneden, C., et al. 2003a, ApJ, 591, 936

Sneden, C., Preston, G. W., \& Cowan, J. J. 2003b, ApJ, 592, 504

Sneden, C., Ivans, I. I., \& Fulbright, J. P. 2004, Origin and Evolution of the Elements, 170

Sneden, C., Cowan, J. J., \& Gallino, R. 2008, ARA\&A, in press

Sommer-Larsen, J., \& Zhen, C. 1990, MNRAS, 242, 10

Sommer-Larsen, J., Beers, T. C., Flynn, C., Wilhelm, R., \& Christensen, P. R. 1997, ApJ, 481, 775

Stanford, L. M., Da Costa, G. S., Norris, J. E., \& Cannon, R. D. 2006, ApJ, 647, 1075

Stephens, A. 1999, AJ, 117, 1771

Stephens, A., \& Boesgaard, A. M. 2002, AJ, 123, 1647
Straniero, O., Gallino, R., \& Cristallo, S. 2006, Nuclear Physics A, 777,311

Tautvaišienè, G., Wallerstein, G., Geisler, D., Gonzalez, G., \& Charbonnel, C. 2004, AJ, 127, 373

Tolstoy, E., Venn, K. A., Shetrone, M., Primas, F., Hill, V., Kaufer, A., \& Szeifert, T. 2003, AJ, 125, 707

Travaglio, C., Gallino, R., Arnone, E., Cowan, J., Jordan, F., \& Sneden, C. 2004, ApJ, 601, 864

Tumlinson, J. 2006, ApJ, 641, 1

Unavane, M., Wyse, R. F. G., \& Gilmore, G. 1996, MNRAS, 278 , 727

Urban, S. E., Zacharias, N., Wycoff, O. G. L. U. S. N., \& Washington, 2. D. C. 2004, VizieR Online Data Catalog, 1294, 0

van Leeuwen, F. 2007, A\&A, 474, 653

Venn, K. A., Irwin, M., Shetrone, M. D., Tout, C. A., Hill, V., \& Tolstoy, E. 2004, AJ, 128, 1177

Wagoner, R. V., Fowler, W. A., \& Hoyle, F. 1967, ApJ, 148, 3

Wallerstein, G. 1962, ApJS, 6, 407

Wanajo, S. 2006, ApJ, 647, 1323

Wanajo, S., \& Ishimaru, Y. 2006, Nuclear Physics A, 777, 676

Wanajo, S. 2007, ApJ, 666, L77

Wasserburg, G. J., Busso, M., \& Gallino, R. 1996, ApJ, 466, L109

Wheeler, J. C., Sneden, C., \& Truran, J. W., Jr. 1989, ARA\&A, 27,279

Wisotzki, L., Christlieb, N., Bade, N., Beckmann, V., Köhler, T., Vanelle, C., \& Reimers, D. 2000, A\&A, 358, 77

Wyse, R. F. G., \& Gilmore, G. 1988, AJ, 95, 1404

Yoshii, Y. 1981, A\&A, 97, 280

Yong, D., Karakas, A. I., Lambert, D. L., Chieffi, A., \& Limongi, M. 2008, ApJ preprint doi:10.1086/592600

Zacharias, N., Urban, S. E., Zacharias, M. I., Wycoff, G. L., Hall, D. M., Monet, D. G., \& Rafferty, T. J. 2004a, AJ, 127, 3043

Zacharias, N., Monet, D. G., Levine, S. E., Urban, S. E., Gaume, R., \& Wycoff, G. L. 2004b, BAAS, 36, 1418 
TABLE 1

Space Velocities and Orbital Parameters for the Stellar Sample

\begin{tabular}{|c|c|c|c|c|c|c|c|c|c|c|c|c|}
\hline Star name & {$[\mathrm{Fe} / \mathrm{H}]$} & $\begin{array}{c}D \\
(\mathrm{pc})\end{array}$ & $\begin{array}{c}U \\
\left(\mathrm{~km} \mathrm{~s}^{-1}\right)\end{array}$ & $\begin{array}{c}V \\
\left(\mathrm{~km} \mathrm{~s}^{-1}\right)\end{array}$ & $\begin{array}{c}W \\
\left(\mathrm{~km} \mathrm{~s}^{-1}\right)\end{array}$ & $\begin{array}{c}V_{\phi} \\
\left(\mathrm{km} \mathrm{s}^{-1}\right)\end{array}$ & $\begin{array}{l}R_{\text {peri }} \\
(\mathrm{kpc})\end{array}$ & $\begin{array}{l}R_{\text {apo }} \\
(\mathrm{kpc})\end{array}$ & $e$ & $\begin{array}{c}\left|Z_{\max }\right| \\
(\mathrm{kpc})\end{array}$ & $\begin{array}{c}J_{X} \\
\left(\mathrm{kpc} \mathrm{km} \mathrm{s}{ }^{-1}\right)\end{array}$ & $\begin{array}{c}J_{Y} \\
\left(\mathrm{kpc} \mathrm{km} \mathrm{s}{ }^{-1}\right)\end{array}$ \\
\hline $\mathrm{BD}+173248$ & -2.05 & 243 & 75 & 99 & -21 & 98 & 7.9 & 20.6 & 0.45 & 0.3 & -13 & 181 \\
\hline CD -38 245 & -4.20 & 4728 & 261 & -129 & -18 & -106 & 2.5 & 36.3 & 0.87 & 17.1 & -587 & -1065 \\
\hline CS $31082-001$ & -2.91 & 2515 & -150 & -256 & -206 & -253 & 1.2 & 20.0 & 0.89 & 17.8 & -661 & 2239 \\
\hline $\mathrm{G} 4-36$ & -1.93 & 244 & 337 & -89 & -81 & -91 & 2.2 & 33.4 & 0.87 & 2.3 & -19 & 648 \\
\hline HD 122563 & -2.79 & 237 & 112 & 38 & 17 & 38 & 6.9 & 14.9 & 0.37 & 0.3 & -8 & -117 \\
\hline HE $1327-2326$ & -5.96 & 1160 & 221 & 246 & 325 & 263 & $\ldots$ & $\ldots$ & $\ldots$ & $\ldots$ & -389 & -2398 \\
\hline HIP 92167 & -1.47 & 162 & 61 & 152 & -284 & 153 & 7.1 & 13.5 & 0.31 & 9.8 & -51 & 2421 \\
\hline HR 2835 & -0.55 & 31 & 62 & 217 & -26 & 217 & 6.9 & 10.2 & 0.19 & 0.2 & -2 & 218 \\
\hline
\end{tabular}

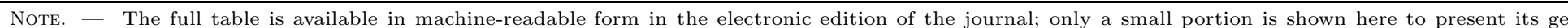

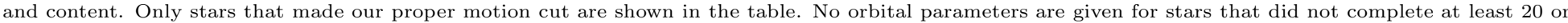
Galactic center. 
TABLE 2

Abundance Ratios for Members of the Inner Halo Population

\begin{tabular}{|c|c|c|c|c|c|c|c|c|c|c|}
\hline Star name & {$[\mathrm{Fe} / \mathrm{H}]$} & {$[\mathrm{Na} / \mathrm{Fe}]$} & {$[\mathrm{Mg} / \mathrm{Fe}]$} & {$[\mathrm{Ca} / \mathrm{Fe}]$} & {$[\mathrm{Ti} / \mathrm{Fe}]$} & {$[\mathrm{Ni} / \mathrm{Fe}]$} & {$[\mathrm{Y} / \mathrm{Fe}]$} & {$[\mathrm{Ba} / \mathrm{Fe}]$} & {$[\mathrm{Eu} / \mathrm{Fe}]$} & Reference \\
\hline HD 221830 & -0.52 & +0.14 & +0.47 & +0.24 & +0.25 & +0.15 & -0.03 & -0.28 & & 1 \\
\hline HIP 96185 & -0.58 & +0.19 & +0.38 & +0.21 & +0.32 & +0.06 & -0.06 & +0.04 & +0.29 & 2 \\
\hline HD 157089 & -0.59 & +0.04 & +0.22 & +0.18 & +0.27 & +0.00 & +0.02 & -0.28 & 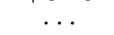 & 1 \\
\hline HIP 86431 & -0.64 & +0.08 & +0.33 & +0.17 & +0.20 & +0.01 & -0.15 & -0.07 & +0.22 & 2 \\
\hline HIP 58357 & -0.65 & +0.04 & +0.31 & +0.18 & +0.32 & +0.03 & $\ldots$ & +0.16 & $\ldots$ & 2 \\
\hline HIP 33582 & -0.74 & +0.06 & +0.47 & +0.24 & +0.41 & 10.00 & $\ldots$ & -0.18 & $\ldots$ & 2 \\
\hline HIP 64115 & -0.74 & +0.15 & +0.47 & +0.27 & +0.29 & +0.09 & +0.04 & -0.01 & +0.39 & 2 \\
\hline HIP 92781 & -0.75 & +0.09 & +0.37 & +0.26 & +0.25 & +0.04 & +0.09 & +0.24 & +0.37 & 2 \\
\hline HIP 74033 & -0.78 & +0.13 & +0.34 & +0.21 & +0.29 & +0.00 & +0.07 & +0.01 & +0.30 & 2 \\
\hline HIP 58962 & -0.80 & -0.04 & +0.11 & +0.06 & +0.01 & -0.07 & -0.19 & -0.18 & & 3 \\
\hline HIP 86013 & -0.82 & +0.14 & +0.39 & +0.28 & +0.34 & +0.04 & +0.00 & -0.07 & +0.37 & 2 \\
\hline HIP 58229 & -0.94 & -0.14 & +0.18 & +0.20 & +0.20 & -0.10 & -0.15 & +0.07 & +0.32 & 2 \\
\hline HIP 66665 & -0.97 & +0.16 & +0.49 & +0.34 & +0.25 & +0.08 & -0.10 & -0.06 & +0.08 & 2 \\
\hline HIP 77946 & -0.97 & +0.21 & +0.55 & +0.36 & +0.35 & +0.09 & +0.00 & +0.05 & +0.24 & 2 \\
\hline HIP 109067 & -0.97 & +0.03 & +0.36 & +0.32 & +0.25 & +0.07 & +0.21 & -0.10 & $\ldots$ & 2 \\
\hline HIP 10449 & -0.98 & -0.04 & +0.42 & +0.28 & +0.30 & +0.01 & +0.08 & +0.10 & $\ldots$ & 2 \\
\hline HIP 5458 & -1.04 & -0.06 & +0.45 & +0.37 & +0.12 & +0.02 & -0.21 & +0.08 & +0.56 & 2 \\
\hline HIP 17666 & -1.10 & -0.05 & +0.46 & +0.29 & +0.36 & +0.05 & +0.55 & +0.44 & $\ldots$ & 2 \\
\hline HIP 44033 & -1.12 & -0.06 & +0.32 & +0.23 & +0.22 & -0.07 & -0.05 & +0.17 & $\ldots$ & 4 \\
\hline HIP 104659 & -1.12 & +0.08 & +0.38 & +0.21 & +0.27 & +0.01 & -0.07 & -0.03 & +0.24 & 2 \\
\hline HIP 98532 & -1.23 & -0.12 & +0.50 & +0.37 & +0.31 & +0.07 & +0.14 & +0.23 & +0.06 & 2 \\
\hline HIP 81170 & -1.26 & -0.11 & +0.41 & $\begin{array}{l}0.27 \\
+0.27\end{array}$ & $\begin{array}{l}0.39 \\
+0.39\end{array}$ & +0.00 & +0.37 & +0.06 & +0.50 & 2 \\
\hline HIP 109390 & -1.34 & -0.05 & +0.50 & +0.23 & +0.29 & -0.08 & +0.04 & +0.01 & +0.39 & 2 \\
\hline HIP 60632 & -1.65 & +0.04 & +0.43 & +0.36 & +0.44 & +0.07 & +0.08 & +0.02 & $\ldots$ & 2 \\
\hline HIP 98020 & -1.67 & -0.29 & +0.26 & $\begin{array}{l}+0.27 \\
\end{array}$ & +0.28 & +0.00 & -0.05 & -0.11 & $\ldots$ & 2 \\
\hline HIP 29992 & -1.71 & -0.08 & +0.33 & +0.28 & +0.25 & -0.03 & $\ldots$ & -0.27 & $\ldots$ & 2 \\
\hline HIP 97468 & -1.71 & +0.01 & +0.57 & +0.44 & +0.35 & +0.01 & -0.18 & +0.00 & +0.27 & 2 \\
\hline HIP 38621 & -1.81 & -0.07 & +0.43 & +0.40 & +0.27 & +0.04 & & -0.33 & $\ldots$ & 2 \\
\hline HIP 103269 & -1.81 & -0.41 & +0.29 & +0.23 & +0.24 & -0.04 & -0.13 & $\begin{array}{l}0.00 \\
+0.00\end{array}$ & $\ldots$ & 2 \\
\hline HIP 68807 & -1.83 & -0.13 & $\begin{array}{l}+0.49 \\
\end{array}$ & +0.37 & +0.20 & -0.03 & -0.30 & -0.05 & +0.40 & 2 \\
\hline HIP 18915 & -1.85 & +0.14 & +0.38 & +0.36 & +0.35 & +0.07 & +0.17 & +0.11 & $\ldots$ & 2 \\
\hline HIP 44124 & -1.96 & $\ldots$ & +0.37 & +0.24 & +0.25 & +0.12 & & +0.10 & $\ldots$ & 2 \\
\hline HIP 14594 & -2.13 & -0.05 & +0.52 & +0.35 & +0.37 & +0.05 & -0.10 & -0.14 & $\ldots$ & 2 \\
\hline HIP 115949 & -2.19 & -0.50 & +0.46 & +0.38 & +0.25 & +0.10 & -0.28 & -0.28 & +0.62 & 2 \\
\hline HIP 60719 & -2.35 & +0.11 & +0.49 & $\begin{array}{l}+0.39 \\
\end{array}$ & +0.24 & $\ldots$ & -0.25 & -0.02 & $\ldots$ & 2 \\
\hline HIP 96115 & -2.41 & +0.08 & +0.58 & +0.51 & +0.49 & +0.23 & +0.08 & -0.16 & $\ldots$ & 2 \\
\hline HIP 72461 & -2.48 & -0.07 & +0.42 & +0.37 & +0.52 & $\ldots$ & $\ldots$ & -0.32 & $\ldots$ & 2 \\
\hline
\end{tabular}
2002 
TABLE 3

Abundance Ratios for Members of the Outer Halo Population

\begin{tabular}{|c|c|c|c|c|c|c|c|c|c|c|}
\hline Star name & {$[\mathrm{Fe} / \mathrm{H}]$} & {$[\mathrm{Na} / \mathrm{Fe}]$} & {$[\mathrm{Mg} / \mathrm{Fe}]$} & {$[\mathrm{Ca} / \mathrm{Fe}]$} & $\overline{[\mathrm{Ti} / \mathrm{Fe}]}$ & 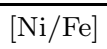 & {$[\mathrm{Y} / \mathrm{Fe}]$} & $\overline{[\mathrm{Ba} / \mathrm{Fe}]}$ & $\overline{[\mathrm{Eu} / \mathrm{Fe}]}$ & Reference \\
\hline HIP 19814 & -0.71 & -0.48 & +0.07 & +0.01 & +0.08 & -0.13 & -0.12 & +0.17 & . & 1 \\
\hline HIP 117041 & -0.88 & +0.12 & +0.53 & +0.32 & +0.30 & +0.09 & -0.02 & -0.10 & $\ldots$ & 2 \\
\hline HIP 57265 & -1.10 & -0.29 & +0.19 & +0.26 & +0.17 & -0.10 & -0.18 & +0.01 & +0.48 & 2 \\
\hline HIP 62747 & -1.54 & +0.00 & +0.56 & +0.40 & +0.26 & +0.02 & +0.00 & +0.08 & +0.26 & 2 \\
\hline HD 20 & -1.58 & $\ldots$ & +0.17 & +0.24 & +0.20 & -0.09 & -0.04 & +0.31 & +0.80 & 3 \\
\hline HIP 5445 & -1.58 & -0.33 & +0.26 & +0.27 & +0.24 & -0.09 & -0.22 & +0.03 & +0.65 & 2 \\
\hline HIP 19797 & -1.68 & +0.09 & +0.34 & +0.32 & +0.38 & +0.00 & +0.00 & +0.04 & $\ldots$ & 2 \\
\hline HE $0447-4858$ & -1.69 & & +0.24 & +0.24 & +0.28 & +0.33 & & +0.45 & $\ldots$ & 3 \\
\hline HIP 15904 & -1.76 & -0.14 & +0.40 & +0.33 & +0.30 & +0.01 & -0.14 & +0.01 & $\ldots$ & 1 \\
\hline HIP 21609 & -1.76 & -0.25 & +0.34 & +0.39 & +0.63 & -0.05 & $\ldots$ & -0.30 & $\ldots$ & 2 \\
\hline HIP 115167 & -1.77 & +0.10 & +0.33 & +0.43 & +0.38 & +0.09 & -0.05 & +0.13 & $\ldots$ & 2 \\
\hline HE $1343-0640$ & -1.90 & & +0.37 & +0.41 & +0.32 & -0.21 & +0.51 & +0.70 & $\ldots$ & 3 \\
\hline G $4-36$ & -1.93 & -0.28 & -0.19 & -0.11 & +0.55 & +0.48 & $\ldots$ & -0.72 & $\ldots$ & 4 \\
\hline CS 29522-046 & -2.09 & & +0.40 & +0.38 & +0.41 & -0.03 & +0.09 & +0.14 & $\ldots$ & 5 \\
\hline HIP 87693 & -2.11 & +0.12 & +0.46 & +0.39 & +0.48 & +0.06 & $\ldots$ & -0.46 & $\ldots$ & 2 \\
\hline HE $0143-1135$ & -2.13 & & +0.33 & +0.26 & +0.24 & -0.09 & .. & -0.07 & $\ldots$ & 3 \\
\hline HD 221170 & -2.15 & $\ldots$ & +0.30 & +0.27 & +0.24 & -0.16 & -0.04 & +0.38 & +0.85 & 3 \\
\hline HE $2329-3702$ & -2.15 & $\ldots$ & +0.31 & +0.44 & +0.42 & -0.22 & $\ldots$ & -0.14 & $\ldots$ & 3 \\
\hline HE $1330-0354$ & -2.29 & $\ldots$ & +0.32 & +0.40 & +0.54 & -0.08 & $\ldots$ & -0.47 & $\ldots$ & 3 \\
\hline HIP 86443 & -2.32 & $\ldots$ & +0.52 & +0.38 & +0.42 & +0.07 & +0.07 & -0.25 & $\ldots$ & 2 \\
\hline HE $1337-0453$ & -2.34 & $\ldots$ & +0.38 & +0.44 & +0.39 & -0.14 & $\ldots$ & -0.24 & $\ldots$ & 3 \\
\hline HE $1256-0651$ & -2.35 & $\ldots$ & +0.22 & +0.32 & +0.30 & +0.06 & $\ldots$ & -0.26 & $\ldots$ & 3 \\
\hline HE $2345-1919$ & -2.46 & $\ldots$ & +0.33 & +0.25 & +0.22 & -0.01 & $\ldots$ & -0.45 & $\ldots$ & 3 \\
\hline $\mathrm{BD}+241676$ & -2.50 & -0.20 & +0.49 & +0.43 & +0.39 & +0.30 & +0.17 & -0.49 & $\ldots$ & 4 \\
\hline HD 186478 & -2.57 & +0.20 & +0.39 & +0.44 & +0.27 & -0.18 & -0.19 & -0.04 & +0.48 & 6 \\
\hline HE $1320-1339$ & -2.78 & $\ldots$ & +0.25 & +0.26 & +0.25 & +0.43 & -0.13 & -0.42 & +0.16 & 3 \\
\hline CS 29491-069 & -2.81 & $\ldots$ & +0.28 & +0.29 & +0.20 & -0.05 & +0.00 & +0.34 & +1.06 & 3 \\
\hline CS 29495-041 & -2.82 & +0.24 & +0.33 & +0.38 & +0.24 & +0.02 & -0.41 & -0.65 & -0.09 & 6 \\
\hline BS $16085-050$ & -2.91 & $\ldots$ & +0.61 & +0.38 & +0.22 & +0.20 & $\ldots$ & -1.56 & $\ldots$ & 7 \\
\hline HE $0547-4539$ & -3.01 & $\ldots$ & +0.13 & +0.13 & +0.19 & +0.03 & -0.33 & -1.03 & $\ldots$ & 3 \\
\hline CS 22169-035 & -3.04 & $\ldots$ & +0.09 & +0.13 & -0.08 & -0.28 & -0.38 & -1.19 & $\ldots$ & 6 \\
\hline $\mathrm{BD}-185550$ & -3.06 & +0.05 & +0.31 & +0.41 & +0.14 & -0.05 & $\ldots$ & -0.74 & -0.20 & 6 \\
\hline HE $0454-4758$ & -3.10 & $\ldots$ & +0.29 & +0.28 & +0.32 & +0.07 & -0.11 & -0.69 & $\ldots$ & 3 \\
\hline CS 29502-092 & -3.18 & $\ldots$ & +0.42 & +0.31 & +0.19 & +0.08 & -0.63 & -1.26 & $\ldots$ & 5 \\
\hline CS 30325-094 & -3.30 & +0.09 & +0.38 & +0.38 & +0.28 & +0.04 & $\ldots$ & -1.88 & $\ldots$ & 6 \\
\hline HE $1337+0012$ & -3.44 & $\ldots$ & +0.55 & +0.48 & +0.51 & +0.17 & $\ldots$ & +0.07 & $\ldots$ & 3 \\
\hline HE $1351-1049$ & -3.45 & $\ldots$ & +0.30 & +0.32 & +0.30 & +0.30 & $\ldots$ & +0.13 & $\ldots$ & 3 \\
\hline CS 22172-002 & -3.86 & -0.35 & +0.20 & +0.37 & +0.21 & -0.15 & -0.98 & -1.17 & $\ldots$ & 6 \\
\hline CD -38 245 & -4.20 & -0.06 & +0.20 & +0.20 & +0.28 & -0.19 & -0.45 & -0.76 & $\ldots$ & 6 \\
\hline
\end{tabular}

REFEREnCes. - (1) Stephens \& Boesgaard 2002; (2) Fulbright| 2000; (3) Barklem et al. 2005; (4) Ivans et al. 2003; (5) Lai et al. 2008; (6) Cayrel et al.|2004, François et al. 2007; (7) Honda et al. 2004b 
TABLE 4

Space Velocities and Orbital Parameters for the Globular Cluster Sample

\begin{tabular}{|c|c|c|c|c|c|c|c|c|c|c|c|c|}
\hline Cluster & $\langle[\mathrm{Fe} / \mathrm{H}]\rangle$ & $\begin{array}{c}D \\
(\mathrm{kpc})\end{array}$ & $\begin{array}{c}U \\
\left(\mathrm{~km} \mathrm{~s}^{-1}\right)\end{array}$ & $\begin{array}{c}V \\
\left(\mathrm{~km} \mathrm{~s}^{-1}\right)\end{array}$ & $\begin{array}{c}W \\
\left(\mathrm{~km} \mathrm{~s}^{-1}\right)\end{array}$ & $\begin{array}{c}V_{\phi} \\
\left(\mathrm{km} \mathrm{s}^{-1}\right)\end{array}$ & $\begin{array}{l}R_{\text {peri }} \\
(\mathrm{kpc})\end{array}$ & $\begin{array}{l}R_{\text {apo }} \\
(\mathrm{kpc})\end{array}$ & $e$ & $\begin{array}{c}\left|Z_{\max }\right| \\
(\mathrm{kpc})\end{array}$ & $\begin{array}{c}J_{X} \\
\left(\mathrm{kpc} \mathrm{km} \mathrm{s}^{-1}\right)\end{array}$ & $\begin{array}{c}J_{Y} \\
\left(\mathrm{kpc} \mathrm{km} \mathrm{s}^{-1}\right)\end{array}$ \\
\hline NGC 288 & -1.39 & 8.8 & 16 & -27 & 53 & -27 & 5.0 & 14.1 & 0.48 & 13.5 & -238 & -1035 \\
\hline NGC 362 & -1.33 & 8.5 & 21 & -58 & -80 & -51 & 1.5 & 51.9 & 0.95 & 47.9 & 40 & 1478 \\
\hline NGC 1904 (M79) & -1.42 & 12.9 & 120 & 32 & 71 & 103 & 6.1 & 18.8 & 0.51 & 6.4 & -388 & -1423 \\
\hline NGC 2298 & -1.90 & 10.7 & -62 & 12 & 97 & -27 & 3.8 & 16.4 & 0.62 & 3.2 & -876 & -1046 \\
\hline NGC 4590 (M68) & -2.34 & 10.2 & 188 & 254 & 5 & 301 & 2.0 & 33.0 & 0.89 & 26.0 & -1560 & 1022 \\
\hline NGC 5272 (M3) & -1.50 & 10.4 & -17 & 104 & -126 & 105 & 5.3 & 16.9 & 0.52 & 16.8 & -1237 & 2158 \\
\hline NGC 5466 & -2.05 & 15.9 & 248 & 81 & 207 & 44 & 9.0 & 23.1 & 0.44 & 23.0 & -610 & -432 \\
\hline NGC 5897 & -1.84 & 12.4 & 30 & -81 & 118 & -77 & 7.4 & 12.7 & 0.26 & 7.6 & 136 & -3024 \\
\hline NGC 5904 (M5) & -1.30 & 7.5 & -323 & 80 & -204 & 84 & 3.9 & 9.7 & 0.43 & 8.0 & -493 & 2764 \\
\hline NGC 6093 (M80) & -1.73 & 10.0 & -12 & -65 & -81 & -65 & 6.5 & 8.7 & 0.15 & 3.4 & 312 & 2099 \\
\hline NGC 6121 (M4) & -1.18 & 2.2 & -57 & 27 & -8 & 26 & 0.1 & 1.0 & 0.88 & 0.7 & -14 & 119 \\
\hline NGC 6218 (M12) & -1.36 & 4.9 & -50 & 122 & -106 & 125 & 2.0 & 5.0 & 0.43 & 3.0 & -396 & 2137 \\
\hline NGC 6254 (M10) & -1.52 & 4.4 & -84 & 133 & 97 & 137 & 1.6 & 4.1 & 0.43 & 2.3 & -119 & -2170 \\
\hline NGC 6362 & -1.04 & 7.6 & 81 & 108 & 37 & 121 & 2.9 & 9.6 & 0.54 & 3.0 & 97 & -1034 \\
\hline NGC 6397 & -2.02 & 2.3 & 34 & 129 & -99 & 130 & 0.1 & 1.5 & 0.93 & 0.7 & 144 & 1874 \\
\hline NGC 6528 & -0.06 & 7.9 & 27 & -26 & -227 & -26 & 5.0 & 6.0 & 0.09 & 0.6 & -61 & 5636 \\
\hline NGC 6553 & -0.28 & 6.0 & 9 & 215 & 14 & 215 & 3.7 & 4.6 & 0.10 & 0.3 & 72 & -323 \\
\hline NGC 6656 (M22) & -1.49 & 3.2 & 152 & 195 & -118 & 191 & 0.8 & 1.7 & 0.38 & 0.5 & 19 & 2311 \\
\hline NGC 6752 & -1.54 & 4.0 & 36 & 197 & 24 & 199 & 1.0 & 4.4 & 0.63 & 2.6 & 301 & -548 \\
\hline NGC 6809 (M55) & -1.88 & 5.3 & -202 & 115 & -181 & 121 & 2.9 & 4.8 & 0.25 & 2.6 & 115 & 4370 \\
\hline NGC 6838 (M71) & -0.76 & 4.0 & -77 & 162 & -2 & 173 & 0.1 & 5.6 & 0.96 & 0.4 & 42 & 62 \\
\hline NGC 7078 (M15) & -2.38 & 10.3 & -148 & 1 & -58 & 56 & 1.4 & 14.0 & 0.81 & 5.1 & -477 & 1908 \\
\hline NGC 7099 (M30) & -2.31 & 8.0 & 68 & -80 & 60 & -87 & 3.1 & 11.8 & 0.58 & 10.1 & -322 & -1715 \\
\hline Pal 5 & -1.31 & 23.2 & 10 & -42 & -45 & -42 & 19.0 & 21.9 & 0.07 & 16.7 & 692 & 1661 \\
\hline Pal 12 & -0.75 & 19.1 & -225 & -109 & -21 & -55 & 11.0 & 21.9 & 0.33 & 17.5 & -1673 & 3763 \\
\hline
\end{tabular}

